Supplemental Information for:

\title{
Characterization of tailoring steps of nargenicin A1 biosynthesis reveals novel analogue with anticancer
} activities

Dipesh Dhakal ${ }^{1}$, Jang Mi Han¹, Ravindra Mishra ${ }^{1}$, Ramesh Prasad Pandey ${ }^{1}$, Tae-Su Kim¹, Vijay Rayamajhi' ${ }^{1}$, Hye Jin Jung ${ }^{1,2}$, Tokutaro Yamaguchi1,2, Jae Kyung Sohng ${ }^{1,2, *}$

1Department of Life Science and Biochemical Engineering, SunMoon University, 70 Sunmoon-ro 221, Tangjeong-myeon, Asan-si, Chungnam 31460, Republic of Korea

${ }^{2}$ Department of Pharmaceutical Engineering and Biotechnology, SunMoon University, 70 Sunmoon-ro 221, Tangjeongmyeon, Asan-si, Chungnam 31460, Republic of Korea.

${ }^{*}$ Corresponding author:

Prof. Jae Kyung Sohng

Tel: +82(41)530-2246

Email: sohng@sunmoon.ac.kr

Fax: +82(41)530-8229 


\section{Table of Contents}

\section{S4-S6: Supplemental materials and methods}

S7-S8: Table S1. Putative gene functions in Nargenicin A1 biosynthetic gene cluster

S9: Table S2. The nargenicin A1 gene cluster reported from three different Nocardia sps.

S10-S11: Table S3. List of strains and plasmids used in this study

S12-S13: Table S4. List of oligonucleotides used in the study

S14: Table S5. Antibacterial activities of diverse nargenicin analogues against different pathogens

S15: Table S6. Anti-cancer $\left(\mathrm{IC}_{50}(\mu \mathrm{M})\right)$ potential of nargenicin analogues against different cancer cell lines

S16: Figure S1. Metabolite profiling of Nocardia sp. CS682 based on analysis of HPLC chromatogram of ethyl acetate extract (a) and respective structures(b)

S17: Figure S2. HR-QTOF Mass chromatogram and UV absorbance of different compounds from Nocardia sp. CS682

S18-S23: Figure S6. Different NMR spectrum of nargenicin A1 (1)

S24-S33: Figure S4. Different NMR spectrum of nodusmicin (2)

S34-S45: Figure S5. Different NMR spectrum of 18-O-acetyl nargenicin (13)

S46-S50: Figure S6. Different NMR spectrum of 18-O-acetyl nodusmicin (12)

S51: Figure S7. Analysis of heterologous expression of nargenicin A1

S52: Figure S8. Mass analysis for nargenicin A1 from heterologous production host

S53: Figure S9. Confirmation of production of nargenicin A1 in heterologous host based on mass fragmentation pattern 
S54: Figure S10. Construction of ngnP1 deletion mutant, Nocardia sp. $\Delta$ NgnP1

S55: Figure S11. HPLC chromatogram of extract from different strains.(a) Wild type, Nocardia sp. CS682, (b) deletion mutant Nocardia sp. $\Delta \mathrm{NgnP} 1$ and (c)complementation strain Nocardia sp. $\Delta \mathrm{NgnP} 1 / \mathrm{p} 18 \mathrm{NgnP} 1$

S56: Figure S12. HR-QTOF Mass chromatogram of compound 6 from Nocardia sp. $\Delta \mathrm{NgnP} 1$

S57: Figure S13. HR-QTOF Mass chromatogram of compound 7 from Nocardia sp. $\Delta \mathrm{NgnP} 1$

S58: Figure S14. Construction of ngnM deletion mutant, Nocardia sp. $\Delta \mathrm{NgnM}$

S59: Figure S15. HPLC chromatogram of extract from different strains (a) Wild type, Nocardia sp. CS682, (b) deletion mutant Nocardia sp. $\Delta \mathrm{NgnM}$ and (c) complementation strain Nocardia sp. $\Delta \mathrm{NgnM} / \mathrm{p} 18 \mathrm{NgnM}$

S60: Figure S16. HR-QTOF Mass chromatogram and UV absorbance of compounds from Nocardia sp. $\Delta N g n M$

S61-S72: Figure S17. Different NMR spectrum of 23-demethyl 8, 13 deoxynodusmicin (8)

S73-S81: Figure S18. Different NMR spectrum of 23 demethyl 8, 13 deoxynargenicin (9)

S82: Figure S19. SDS Page of purified proteins (a) NgnM and (b) NgnO3

S83: Figure S20. Reaction of different substrates with NgnM

S84: Figure S21. HR-QTOF Mass chromatogram of different compounds generated by enzyme reaction with NgnM

S85-S88: Figure S22. Different NMR spectrum of 8, 13 deoxynodusmicin (10)

S89-S95: Figure S23. Different NMR spectrum of 8, 13 deoxynargenicin (11)

S96: Figure S24. Reaction of different substrates with $\mathrm{NgnO} 3$

S97: Figure S25. Construction of ngnN4 deletion mutant, Nocardia sp. $\Delta \mathrm{NgnN} 4$

S98: Figure S26. HPLC chromatogram of extract from different strains (a) Wild type, Nocardia sp. CS682, (b) deletion mutant Nocardia sp. $\Delta \mathrm{NgnM}$ and (c) complementation strain Nocardia sp. $\Delta \mathrm{NgnM} / \mathrm{p} 18 \mathrm{NgnM}$

\section{S99. References}




\section{Supplemental materials and methods}

\section{A. Isolation of compounds 8 and 9}

For the isolation of compounds 8 and $9,500 \mu \mathrm{L}$ Nocardia sp. $\Delta \mathrm{M}$ from stock solution maintained at -80 refrigerator was inoculated in $100 \mathrm{~mL}$ of $\mathrm{BHI}$ medium. The cultivation was carried at $37^{\circ} \mathrm{C}, 200 \mathrm{rpm}$ for $48 \mathrm{~h}$. A total of $8 \mathrm{~L}$ fermentation was performed by inoculating $5 \mathrm{~mL}$ of seed culture into $2 \mathrm{~L}$ Erlenmeyer flask containing $400 \mathrm{~mL}$ of the production medium, DD medium ${ }^{1}$. The $8 \mathrm{~L}$ culture broth was separated to supernatant and cell pellet by centrifugation. The cell pellet was washed two times with acetone, and after removal of acetone by evaporation, the extract was mixed to the supernatant. The culture broth was extracted three times with twice volume of ethyl acetate maintained at $\mathrm{pH} 3$. The ethyl acetate extract was concentrated at reduced pressure to generate crude extract $(2.9 \mathrm{~g})$. The crude extract was dissolved in $100 \mathrm{~mL}$ chilled methanol and filtered with $0.2 \mu \mathrm{M}$ syringe filter. $2 \mathrm{~mL}$ of sample was used for each round of purification of the target compound by semi prep-HPLC with C18 column (YMC-Pack ODS-AQ-HG, 250x20 mm, $10 \mu \mathrm{m}$, Kyoto, Japan) connected to a UV detector $(220 \mathrm{~nm})$. The program for purification was binary conditions with water $(0.025 \%$ trifluoroacetic acid) and acetonitrile: acetonitrile concentration $10 \%$ (0-5 min), $30 \%$ (5-15 min), $60 \%$ (15-25 min), $70 \%$ (25-35 min), 90 \% (35$40 \mathrm{~min}$ ), and $10 \%$ (40-45 min) and finally stop at $46 \mathrm{~min}$ with a flow rate of $10 \mathrm{~mL} / \mathrm{min}$. The targeted peaks corresponding to compounds were collected, vacuum dried, dissolved in $d 6$-DMSO and analyzed by NMR analysis. After confirmation of purity of the compounds, the compounds were utilized by further enzymatic assays.

\section{B. Isolation of compounds 10 and 11}

For the isolation of compounds 10 and 11, the large-scale enzymatic reaction of NgnM was performed with 8 and 9 , respectively. The reaction mixture containing $50 \mathrm{mg} \mathrm{NgnM,} 5 \mu \mathrm{M} \mathrm{SAM}$, substrates ( $5 \mathrm{mM} 8$ or $2 \mathrm{mM} 9$ ), $50 \mathrm{mM}$ Tris buffer, 
$\mathrm{pH} 7.4$ in a final volume of $100 \mathrm{~mL}$ was transferred in $500 \mathrm{~mL}$ Erlenmeyer flask. The flask was incubated in waterbath at at $30^{\circ} \mathrm{C}, 100 \mathrm{rpm}$ for $2 \mathrm{~h}$. The reaction was quenched by adding twice volume of ethyl acetate. The reaction mixture alongwith ethylacetate was transferred to separatory funnel and shaked. The upper organic fraction was collected, dried under vacuum and dissolved in methanol to generate the crude extract. The extract was subjected to purification by semi-prep HPLC and the peak corresponding to 10 and 11 were collected, vacuum dried, dissolved in d6-DMSO and subjected to NMR analysis. After confirmation of purity of the compounds, the compounds were utilized by further enzymatic assays.

\section{Optimization of conditions for capture and cloning of BGC of 1}

The basic experimental procedures were exactly same as standard protocols reported previously ${ }^{2}$.For construction of pathway-specific capture vector, 18bp homologous sequence to genomic DNA is selected as capture arm previously. However, in our case we failed to confirm the cloning of gene cluster in our early attempts using 18bp homologous sequence on each end. Hence, we decided to increase the length of capture arm to 199bp homologous sequence on each end. In addition, E. coli EPI-400 was used for initial transformation after initial extraction of DNA from yeast. By this approach we could obtain positive results in $\sim 80 \%$ (8 out of 10) cases, which was confirmed by PCR amplification from end region of nar BGC. Further one of the pCAP03-nar showing amplification products for genes was selected for gene sequencing and cloning of entire nar BGC was confirmed. The confirmed pCAP03-nar was transferred to E. coli ET host and used for triparental conjugation.

\section{Optimization of culture conditions for heterologous production of 1}

Different actinomycetes strains producing diverse polyketide compounds as Streptomyces venezuelae (pikromycin), Streptomyces coelicolor (actinorhodin), Sachharopolyspora erythea (erythromycin), Amycolatopsis mediterranei and Actinomadure hibisca (pradimicin) were used for primary screening of suitable heterologous host for production of 1.The 
production of 1 was detected in Streptomyces venezuelae (pikromycin), Sachharopolyspora erythea (erythromycin) and Amycolatopsis mediterranei by transfer of nar BGC. However, the clean host without any contaminating secondary metabolites are preferred microbial platform for heterologous expression, whereas Streptomyces venezuelae YJ028 was only clean host available in our laboratory. So, the nar BGC was transferred to Streptomyces venezuelae YJ028 to generate Streptomyces venezuelae YJ028/pCAP03-nar. Further, Streptomyces venezuelae YJ028/pCAP03-nar was cultivated in different mediums as R2YE, BHI, DD and DD1. Among all the medium, the highest yield of 1 was obtained in DD1 medium. 
Table S1. Putative gene functions in Nargenicin A1 biosynthetic gene cluster

\begin{tabular}{|c|c|c|c|c|c|}
\hline Gene & $\mathrm{AA}$ & Closest homologue Protein (CHP) & CHP source strain & CHP Accession No & Identity/Similarity \\
\hline Orf1 & 394 & Diguanylate cyclase & Nocardia suismassiliense & WP_107657110.1 & $94 / 95$ \\
\hline Orf2 & 477 & peptidase M14 & Nocardia argentinensis & AXG22423.1 & $96 / 97$ \\
\hline NgnN2 & 258 & keto-acyl ACP-synthase & Nocardia sp. CS682 & AEI59693.1 & 100 \\
\hline NgnN3 & 375 & prolyl carrier protein dehydrogenase & Nocardia sp. CS682 & AEI59692.1 & 100 \\
\hline NgnN4 & 501 & proline adenyltransferase & Nocardia sp. CS682 & AEI59690.1 & 100 \\
\hline NgnN5 & 91 & proline carrier protein & Nocardia sp. CS682 & AEI59691.1 & 100 \\
\hline $\mathrm{NgnH}$ & 125 & $\begin{array}{l}\text { LuxR response regulator transcription } \\
\text { factor }\end{array}$ & Nocardia tenerifensis & WP_083895053.1 & $71 / 79$ \\
\hline NgnJ & 315 & LysR family transcriptional regulator & Nocardia argentinensis & AXG22418.1 & $95 / 97$ \\
\hline Ngnl & 308 & Retinol dehydrogenase & Nocardia argentinensis & AXG22417.1 & $90 / 95$ \\
\hline $\mathrm{NgnO} 3$ & 274 & Phytanoyl-CoA dioxygenase & Nocardia argentinensis & AXG22416.1 & $98 / 99$ \\
\hline NgnP2 & 405 & Cytochrome P450 & Nocardia argentinensis & AXG22415.1 & $96 / 97$ \\
\hline $\mathrm{NgnF}$ & 65 & ferredoxin & Nocardia argentinensis & AXG22414.1 & $98 / 98$ \\
\hline NgnM & 278 & methyltransferase [ & Nocardia argentinensis & AXG22413.1 & $98 / 99$ \\
\hline NgnG & 280 & $\begin{array}{l}\text { short-chain dehydrogenase } \\
\text { 3-oxoacyl-ACP reductase }\end{array}$ & Nocardia argentinensis & AXG22412.1 & $93 / 96$ \\
\hline NgnO1 & 310 & $\begin{array}{l}\text { LLM class flavin-dependent } \\
\text { oxidoreductase }\end{array}$ & Nocardia argentinensis & AXG22411.1 & $93 / 95$ \\
\hline $\mathrm{NgnO} 2$ & 421 & pristinamycin IIA synthase subunit $A$ & Nocardia argentinensis & AXG22410.1 & $97 / 98$ \\
\hline NgnP1 & 420 & cytochrome P450 & Nocardia argentinensis & AXG22409.1 & $95 / 96$ \\
\hline NgnK & 348 & L-glyceraldehyde 3-phosphate & Nocardia argentinensis & AXG22408.1 & $96 / 98$ \\
\hline
\end{tabular}




\begin{tabular}{|c|c|c|c|c|c|}
\hline & & reductase & & & \\
\hline NgnA & 5959 & type I polyketide synthase & Nocardia argentinensis & AXG22407.1 & $90 / 93$ \\
\hline $\mathrm{NgnB}$ & 4761 & type I polyketide synthase & Nocardia argentinensis & AXG22406.1 & $90 / 94$ \\
\hline $\mathrm{NgnC}$ & 3665 & type I polyketide synthase & Nocardia argentinensis & AXG22405.1 & $91 / 94$ \\
\hline NgnD & 152 & $\begin{array}{l}\text { nuclear transport factor } 2 \text { family } \\
\text { protein }\end{array}$ & Nocardia argentinensis & AXG22404.1 & $98 / 100$ \\
\hline NgnV & 259 & thioesterase & Nocardia argentinensis & AXG22403.1 & $90 / 93$ \\
\hline $\mathrm{NgnU}$ & 1186 & DNA polymerase III subunit alpha & Nocardia suismassiliense & WP_107657112.1 & 99/99 \\
\hline Orf3 & 98 & hypothetical protein & Nocardia argentinensis & AXG22401.1 & $87 / 89$ \\
\hline Orf4 & 273 & phytanoyl-CoA dioxygenase & Nocardia suismassiliense & WP_107660552.1 & $89 / 91$ \\
\hline
\end{tabular}


Table S2. The nargenicin A1 gene cluster reported from three different Nocardia sps.

\begin{tabular}{|l|l|l|l|}
\hline $\begin{array}{l}\text { Nocardia } \\
\text { sp. CS682 }\end{array}$ & $\begin{array}{l}\text { Nocardia argentinensis } \\
\text { ATCC 31306 (Accession } \\
\text { No) }\end{array}$ & $\begin{array}{l}\text { Nocardia arthritidis } \\
\text { isolate (ID: } \\
\text { AUSMDU00012717) }\end{array}$ & Proposed Function \\
\hline Orf1 & Orf2 (AXG22424.1) & - & Diguanylate cyclase \\
\hline Orf2 & Orf3 (AXG22423.1) & - & Peptidase M14 \\
\hline NgnN2 & NgnN2 (AXG22422.1) & NgnN1 & Keto-acyl ACP-synthase \\
\hline NgnN3 & NgnN3 (AXG22421.1) & NgnN3 & $\begin{array}{l}\text { Prolyl carrier protein } \\
\text { dehydrogenase }\end{array}$ \\
\hline NgnN4 & NgnN4 (AXG22420.1) & NgnN4 & Proline adenyltransferase \\
\hline NgnN5 & NgnN5 (AXG22419.1) & NgnN5 & Proline carrier protein \\
\hline NgnH & - & - & Transcriptional regulator \\
\hline NgnJ & NgnJ (AXG22418.1) & NarP & Transcriptional regulator \\
\hline Ngnl & NgnI (AXG22417.1) & NarO & Dehydrogenase \\
\hline NgnO3 & NgnO3 (AXG22416.1) & NarN & Dioxygenase \\
\hline NgnP2 & NgnP2 (AXG22415.1) & NarM & Cytochrome P450 \\
\hline NgnF & NgnF (AXG22414.1) & NarL & Ferredoxin \\
\hline NgnM & NgnM (AXG22413.1) & NarK & O-methyltransferase \\
\hline NgnG & NgnG (AXG22412.1) & NarJ & Short-chain dehydrogenase \\
\hline NgnO1 & NgnO1 (AXG22411.1) & NarO1 & Flavin utilizing monoxygenase \\
\hline NgnO2 & NgnO2 (AXG22410.1) & NarO2 & Flavin utilizing monoxygenase \\
\hline NgnP1 & NgnP1 (AXG22409.1) & NarG & Cytochrome P450 \\
\hline NgnK & NgnK (AXG22408.1) & NarF & Aldoketoreductase \\
\hline NgnA & NgnA (AXG22407.1) & NarA & Polyketide synthase \\
\hline NgnB & NgnB (AXG22406.1) & NarB & Polyketide synthase \\
\hline NgnC & NgnC (AXG22405.1) & NarC & Polyketide synthase \\
\hline NgnD & NgnD (AXG22404.1) & NarD & Bispericyclase \\
\hline NgnV & NgnV (AXG22403.1) & NarE & Thioesterase \\
\hline NgnU & Orf4 (AXG22402.1) & NarR & Resistance \\
\hline & & & \\
\hline
\end{tabular}


Table S3. List of strains and plasmids used in this study

\begin{tabular}{|c|c|c|}
\hline $\begin{array}{c}\text { Bacterial strains, plasmids and } \\
\text { rDNAs }\end{array}$ & Relevant characteristics & Sources \\
\hline \multicolumn{3}{|l|}{ Bacterial strains } \\
\hline E. coli XL1Blue MRF & $\begin{array}{l}\Delta(m c r A) 183 \Delta(m c r C B-h s d S M R-m r r) 173 \text { endA1 supE44 thi-1 recA1 gyrA96 } \\
\text { relA1 lac }\end{array}$ & Stratagene, USA \\
\hline E. coli EPI-400 & Host for maintaining large gene clusters( tonA $\Delta p c n B$ dhfr) & Lucigen,USA \\
\hline E. coli ET-12567 & DNA demethylating strain (dam $\left.{ }^{-} \mathrm{dcm}^{-} h s d S \mathrm{~cm}^{R}\right)$ & John Innes Center, UK \\
\hline E. coliET12567/pUB307 & $\begin{array}{l}\text { Strain with self-transmissible plasmid that mobilises other plasmids into } \\
\text { Streptomyces host }\end{array}$ & John Innes Center, UK \\
\hline E. coli BL21-DE3 & ompT hsdSB (rB-mB-) gal dcm (DE3) & Invitrogen, USA \\
\hline $\begin{array}{l}\text { Saccharomyces } \\
\text { cerevisiae VL6-48N }\end{array}$ & $\begin{array}{l}\text { MAT } \alpha, \text { his3-D200, trp1- } \Delta 1, \text { ura3- } \Delta 1, \text { lys2, ade2- } \\
\text { 101, met14, psi+cirO }\end{array}$ & Kouprina et al. 2008 , \\
\hline E. coli-ngnM & E.coli BL21 containing pET-NgnM & This study \\
\hline E. coli-ngnO3 & E.coli BL21 containing pET-NgnO3 & This study \\
\hline Nocardia sp. CS682 & Wild type producer of nargenicin A1 & Sohng et al. 2008 \\
\hline Nocardia sp. $\Delta \mathrm{N} 4$ & Mutant strain with inactivation of ngnN4 & This study \\
\hline Nocardia sp. $\Delta \mathrm{M}$ & Mutant strain with inactivation of $n g n M$ & This study \\
\hline Nocardia sp. $\Delta \mathrm{P} 1$ & Mutant strain with inactivation of $n g n P 1$ & This study \\
\hline Nocardia sp. $\Delta \mathrm{M} / \mathrm{p} 18 \mathrm{NgnN} 4$ & Gene complementation strain of ngnN4 & This study \\
\hline Nocardia sp. $\Delta \mathrm{M} / \mathrm{p} 18 \mathrm{NgnM}$ & Gene complementation strain of $n g n M$ & This study \\
\hline Nocardia sp. $\Delta \mathrm{M} / \mathrm{p} 18 \mathrm{NgnP} 1$ & Gene complementation strain of $n g n P 1$ & This study \\
\hline Streptomyces venezuelae YJ028 & S. venezuelae with deletion of entire pikromycin gene cluster & Jung et al. 2007 \\
\hline S venezuelae YJ028/pCAP03 & S. venezuelae YJ028 harboring pCAP03 & This study \\
\hline S venezuelae YJ028/pCAP03-nar & Heterologous production host harboring pCAP03-nar & This study \\
\hline \multicolumn{3}{|l|}{ Plasmids and rDNAs } \\
\hline pGEM ${ }^{\circledR}$-T Easy & $\mathrm{T}_{7}$ and SP6 promoters, ColE1 ori, lacZ, $A m p^{\mathrm{R}}$ & Promega, USA \\
\hline pKC1139 & Multi copy E. coli-Streptomyces shuttle vector & Bierman et al. 1992 \\
\hline pULVK2A & Nocardia-E. coli shuttle vector, neor, apr & Kelly and Townsend, 2005 \\
\hline pNV18L2 & Nocardia-E. coli shuttle vector, neor ${ }^{r}$ & Dhakal et al. 2016 \\
\hline pET32a $(+)$ & Single $\mathrm{T}_{7}$ promotor, pBR322 ori, AmpR & Novagen, Germany \\
\hline pCAP03 & Platform vector for TAR. & Addgene plasmid \#69862 \\
\hline pCAP03-nar & pCAP03 harboring nargenicn BCG of Nocardia sp. CS682 & This study \\
\hline pET-NgnM & pET32 containing ngnM & This study \\
\hline pET-NgnO3 & pET32 containing ngnO3 & This study \\
\hline pDNgnN4 & pULVK2A containing deletion cassette for ngnN4 & This study \\
\hline pDNgnM & pULVK2A containing deletion cassette for ngnM & This study. \\
\hline
\end{tabular}




\begin{tabular}{lll}
\hline pDNgnP1 & pULVK2A containing deletion cassette for $n g n P 1$ & This study. \\
p18NgnN4 & pNV18L2 for expression of $n g n N 4$ for complementation & This study. \\
p18NgnM & pNV18L2 for expression of $n g n M$ for complementation & This study. \\
p18NgnP1 & pNV18L2 for expression of $n g n P 1$ for complementation & This study. \\
\hline
\end{tabular}


Table S4. List of oligonucleotides used in the study

\begin{tabular}{|c|c|c|c|}
\hline Gene & Sequence (5'-3') & RS & Notes \\
\hline Del-ngnN4-up-F & ATCGGATCCCAACGCACTAGACCGAAG & BamHI & \multirow{2}{*}{$\begin{array}{l}\text { For amplification of upstream region for deletion of } \\
n g n N 4\end{array}$} \\
\hline Del-ngnN4-up-R & TATTCTAGAGGTCATCGCACCCAGCGG & $X b a l$ & \\
\hline Del-ngnN4-dn-F & TATTCTAGAGCTACATGGTCCCGGACG & Xbal & \multirow{2}{*}{$\begin{array}{l}\text { For amplification of downstream region for deletion of } \\
n g n N 4\end{array}$} \\
\hline$D e l-n g n N 4-d n-R$ & GCCAAGCTTAGATTCAATGACCATGTGGCG & HindlII & \\
\hline Del-ngnM-up-F & GGCGGATCCTCGCACAAAGGTTTAATG & BamHI & \multirow[t]{2}{*}{ For amplification of upstream region for deletion of $n g n M$} \\
\hline Del-ngnM-up-R & TATTCTAGACTCGGGCTCGGCGACGA & Xbal & \\
\hline Del-ngnM-dn-F & TGTCTAGACGACTGGCGATCGCCGAT & Xbal & \multirow{2}{*}{$\begin{array}{l}\text { For amplification of downstream region for deletion of } \\
\text { ngnM }\end{array}$} \\
\hline Del-ngnM-dn-R & GCAAGCTTAGACAGGAGCTGATCACCG & HindllI & \\
\hline Del-ngnP1-up-F & ACTAAGCTTGCGCGAAGAGCACGAGGG & HindlII & \multirow{2}{*}{$\begin{array}{l}\text { For amplification of upstream region for deletion of } \\
n g n P 1\end{array}$} \\
\hline Del-ngnP1-up-R & TGATCTAGACCCGAGACGAGGAGAACC & Xbal & \\
\hline Del-ngnP1-dn-F & CCATCTAGAATCCCGTGGAAGGTGCGC & Xbal & \multirow{2}{*}{$\begin{array}{l}\text { For amplification of downstream region for deletion of } \\
n g n P 1\end{array}$} \\
\hline Del-ngnP1-dn-R & CGGGAATTCCGACGGGCGTCGG & EcoRI & \\
\hline$n g n N 4-F$ & AGTGGATCCGTGAACCTGCTCGATGGA & BamHI & \multirow[t]{2}{*}{ For amplification of $n g n N 4$ for complementation } \\
\hline$n g n N 4-R$ & CGGGTACCTCATGTTGTTGTACTCCT & Kpnl & \\
\hline$n g n M 1-F$ & AGTGGATCCATGCCGCCTTGGCACTTC & $\mathrm{BamHI}$ & \multirow[t]{2}{*}{ For amplification of $n g n M$ for complementation } \\
\hline$n g n M 1-R$ & CGGGTACCTCAGCCACGAGTGGCGGT & Kpnl & \\
\hline$n g n P 1-F$ & CGGGTACCATGAGAATGTCCGATA & Kpnl & \multirow[t]{2}{*}{ For amplification of $n g n P 1$ for complementation } \\
\hline$n g n P 1-R$ & GCATGCATTCAGTGCGACAGCGTC & Nsil & \\
\hline$n g n M 2-F$ & AGTGGATCCATGCCGCCTTGGCACTTC & $\mathrm{BamHI}$ & \multirow[t]{2}{*}{ For amplification of $n g n M$ for protein expression } \\
\hline$n g n M 2-R$ & GCCTCGAGTCAGCCACGAGTGGCGGT & Xhol & \\
\hline$n g n O 3-F$ & CGGGATCCATGCCCAGGATTGACGGGAG & $\mathrm{BamHI}$ & \multirow[t]{2}{*}{ For amplification of $n g n \mathrm{O} 3$ protein expression } \\
\hline ngnO3-R & GCCTCGAGTCACGGCGCGAGACCGAC & Xhol & \\
\hline tsrF & GCCTCGAGTATTCTAGAGCTACATGGTCCCGGAC & & \multirow{2}{*}{$\begin{array}{l}\text { For confirmation of double cross over mutant by PCR of } \\
\text { thiostrepton resistance gene used as selection marker }\end{array}$} \\
\hline$t s r R$ & GCCAAGCTTAGATTCAATGACCATGTGGCG & & \\
\hline aprF & ATGCAATACGAATGGCGA & & \multirow{2}{*}{$\begin{array}{l}\text { For confirmation of loss of vector in double cross over } \\
\text { mutant by PCR of apramycin resistance gene }\end{array}$} \\
\hline aprR & TCAGCCAATCGACTGGCG & & \\
\hline$n g n N 4 C-F$ & AGCGCGACATCATCGCAC & & \multirow[t]{2}{*}{ For confirmation of gene deletion of $n g n N 4$} \\
\hline$n g n N 4 C-R$ & GGTTGGTCAGTTCGGCCT & & \\
\hline$n g n M C-F$ & GACGACCTCTCGTTCAAA & & \multirow[t]{2}{*}{ For confirmation of gene deletion of $n g n M$} \\
\hline$n g n M C-R$ & CAAGAGGGCGCGACTGGAA & & \\
\hline$n g n P 1 C-F$ & TCGAGTGCCTGAGCGTTG & & \multirow[t]{2}{*}{ For confirmation of gene deletion of $n g n P 1$} \\
\hline$n g n P 1 C-R$ & GCCGCACAGTTGAATT & & \\
\hline $\begin{array}{l}\text { nar capture } \\
\text { fragment }\end{array}$ & $\begin{array}{l}\text { CATGGTATAAATAGTGGCTCAGGCGGTGGCCGCA } \\
\text { TCCGGTTCGACAGCGAACAACGGCTCGTCGAACT } \\
\text { CGACCGGACCGCCCTCCGGGCCCAGCACCTCCG }\end{array}$ & & $\begin{array}{l}\text { For insertion in the Pmel digested pCAP03 by Gibson } \\
\text { cloning }\end{array}$ \\
\hline
\end{tabular}




\begin{tabular}{|l|l|l|l|}
\hline CCACCCGTCCGGCGACACCGGCGTGCACCGGGA & & \\
TCATCAGCTTCATTACTTCGACGATCGCCACCTGA & & \\
GTGTTCGGCTCGACATGGTCGCCGACGGCTACGA & & \\
ACGGCGCGGCGCCGGTTTAAACTGGCGGTGCGG & & \\
AATGGCTCGTCACTGTATTCGGGCAAGCGGACGA & & \\
GCGGCTTTGCCAGGTGGTCGATCATGCGGAGC & & \\
ACATCCGGTGCCGGCCACATGATCTCGACACAG & & \\
CGCTCAGCGAGCGCGCGAGAAAACGAGCGGGT & & \\
ACGTGCACGAATCCGTCCTCGATGAACTTTGCGAT & & \\
GTGTTCTGTCTCGAGCATTATGTCGAAAGCTACAT & & \\
A & \\
\hline
\end{tabular}


Table S5. Antibacterial activities of diverse nargenicin analogues against different pathogens

\begin{tabular}{|c|c|c|c|c|c|c|c|c|}
\hline Microorganisms & 1 & 13 & 2 & 12 & 8 & 9 & 10 & 11 \\
\hline \multicolumn{9}{|l|}{ Gram Positive bacteria } \\
\hline S.aureus CCARM 3640(MRSA) & 0.016 & 0.063 & $>32$ & $>32$ & $>32$ & $>32$ & ND & ND \\
\hline S.aureus CCARM 3089 (MRSA) & 0.016 & 0.063 & $>32$ & $>32$ & $>32$ & $>32$ & ND & ND \\
\hline S.aureus CCARM 33591 (MRSA) & 0.016 & 0.125 & $>32$ & $>32$ & $>32$ & $>32$ & ND & ND \\
\hline S.aureus CCARM 3090 (MRSA) & 0.031 & 0.125 & $>32$ & $>32$ & $>32$ & $>32$ & ND & ND \\
\hline S.aureus CCARM 3634 (MRSA) & 0.016 & 0.063 & $>32$ & $>32$ & $>32$ & $>32$ & ND & ND \\
\hline S.aureus CCARM 3635 (MRSA) & 0.031 & 0.125 & $>32$ & $>32$ & $>32$ & $>32$ & ND & ND \\
\hline S.aureus CCARM 0204 (MSSA) & 0.016 & 0.031 & $>32$ & $>32$ & $>32$ & $>32$ & ND & ND \\
\hline S.aureus CCARM 0205 (MSSA) & 0.031 & 0.063 & $>32$ & $>32$ & $>32$ & $>32$ & ND & ND \\
\hline S.aureus CCARM 0027 (MSSA) & 0.031 & 0.125 & $>32$ & $>32$ & $>32$ & $>32$ & ND & ND \\
\hline Kocuria rhizophila ATCC 9341 & 2 & 4 & $>32$ & $>32$ & $>32$ & $>32$ & ND & ND \\
\hline Enterococcus faecalis ATCC 19433 & $>32$ & $>32$ & $>32$ & $>32$ & $>32$ & $>32$ & ND & ND \\
\hline Enterococcus faecali ATCC 1934 & $>32$ & $>32$ & $>32$ & $>32$ & $>32$ & $>32$ & ND & ND \\
\hline \multicolumn{9}{|l|}{ Gram Negative bacteria } \\
\hline Escherichia coli 25922 & $>32$ & $>32$ & $>32$ & $>32$ & $>32$ & $>32$ & ND & ND \\
\hline Pseudomonas aeruginosa KCTC 1637 & $>32$ & $>32$ & $>32$ & $>32$ & $>32$ & $>32$ & ND & ND \\
\hline Salmonella enterica ATCC 14028 & $>32$ & $>32$ & $>32$ & $>32$ & $>32$ & $>32$ & ND & ND \\
\hline Salmonella typhimrium KCTC 1925 & $>32$ & $>32$ & $>32$ & $>32$ & $>32$ & $>32$ & ND & $\mathrm{ND}$ \\
\hline
\end{tabular}

ND: Not determined due to low concentration 
Table S6. Anti-cancer $\left(\mathrm{IC}_{50}(\mu \mathrm{M})\right)$ potential of nargenicin analogues against different cancer cell lines

\begin{tabular}{|l|l|l|l|l|l|l|l|l|l|}
\hline Cell Lines & 1 & 13 & 2 & 12 & 8 & 9 & 10 & 11 \\
\hline \multicolumn{7}{|c|}{ Cancer cell lines } \\
\hline AGS & 154.47 & $>200$ & $>200$ & $>200$ & $>200$ & 8.21 & ND & ND \\
\hline A549 & 102.30 & $>200$ & $>200$ & $>200$ & $>200$ & 9.31 & ND & ND \\
\hline A375SM & $>200$ & $>200$ & $>200$ & $>200$ & $>200$ & 72.03 & ND & ND \\
\hline HCT116 & $>200$ & $>200$ & $>200$ & $>200$ & $>200$ & 48.62 & ND & ND \\
\hline U87MG & $>200$ & $>200$ & $>200$ & $>200$ & $>200$ & 69.93 & ND & ND \\
\hline MCF7 & $>200$ & $>200$ & $>200$ & $>200$ & $>200$ & 83.32 & ND & ND \\
\hline B16F10 & $>200$ & $>200$ & $>200$ & $>200$ & $>200$ & 142.4 & ND & ND \\
\hline HeLa & $>200$ & $>200$ & $>200$ & $>200$ & $>200$ & 54.11 & ND & ND \\
\hline HepG2 & $>200$ & $>200$ & $>200$ & $>200$ & $>200$ & $>200$ & ND & ND \\
\hline Hep3B & 194.01 & $>200$ & $>200$ & $>200$ & $>200$ & 74.01 & ND & ND \\
\hline \multicolumn{7}{|c|}{ Normal cell lines } & & & \\
\hline 267B1 & $>200$ & $>200$ & $>200$ & $>200$ & $>200$ & $>200$ & ND & ND \\
\hline MRC-5 & $>200$ & $>200$ & $>200$ & $>200$ & $>200$ & $>200$ & ND & ND \\
\hline
\end{tabular}


Figure S1. Metabolite profiling of Nocardia sp. CS682 based on analysis of HPLC chromatogram of ethyl acetate extract (a) and respective structures(b)

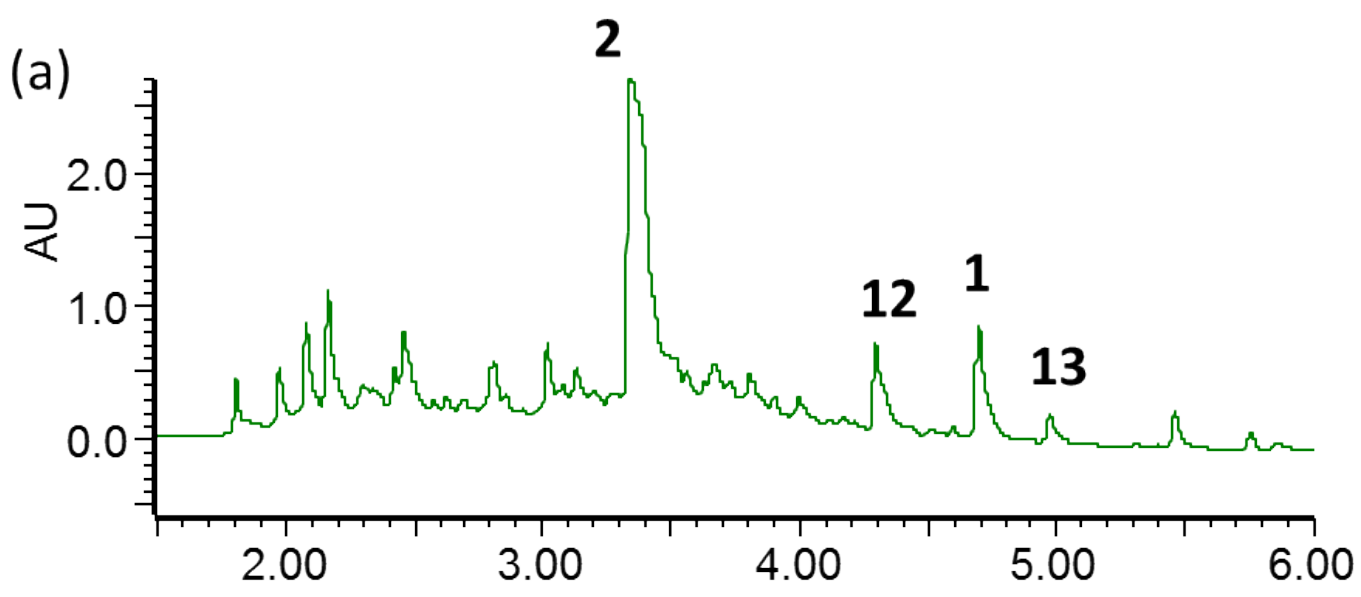

(b)
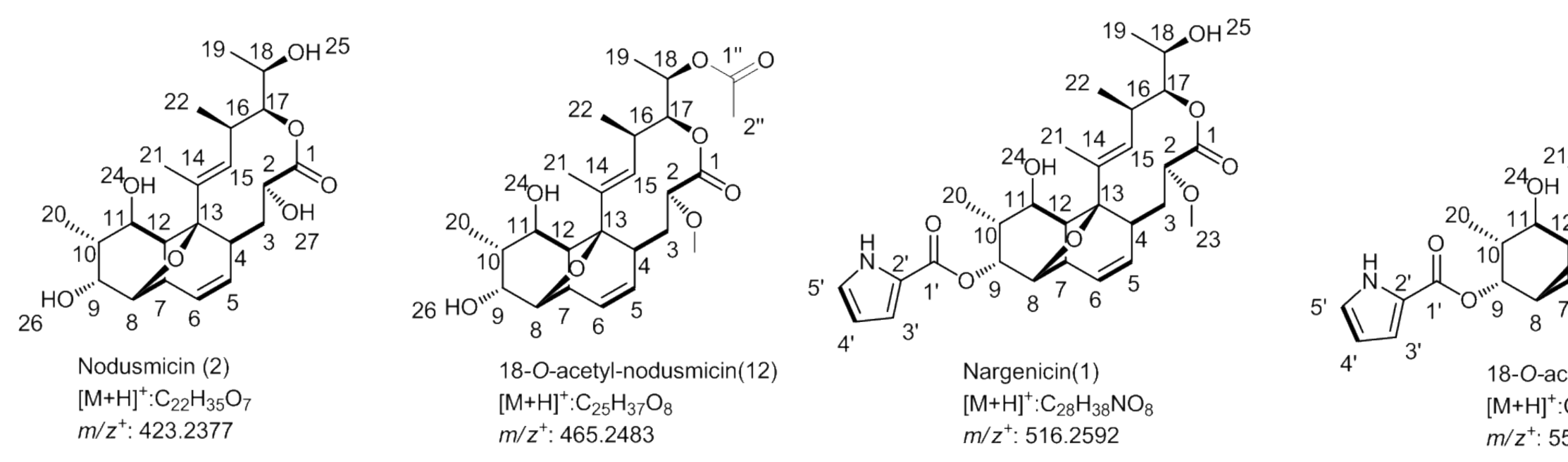
Figure S2. HR-QTOF Mass chromatogram of different compounds from Nocardia sp. CS682, (a)Nodusmicin(2), (b): 18O-acetyl nodusmicin(12),(c) Nargenicin(1) and (d)18-O-acetyl nargenicin(13). UV absorbance of different compounds(e)2, (f) $12, \mathrm{~g}(1)$ and $(\mathrm{h}) 13$.

(a)

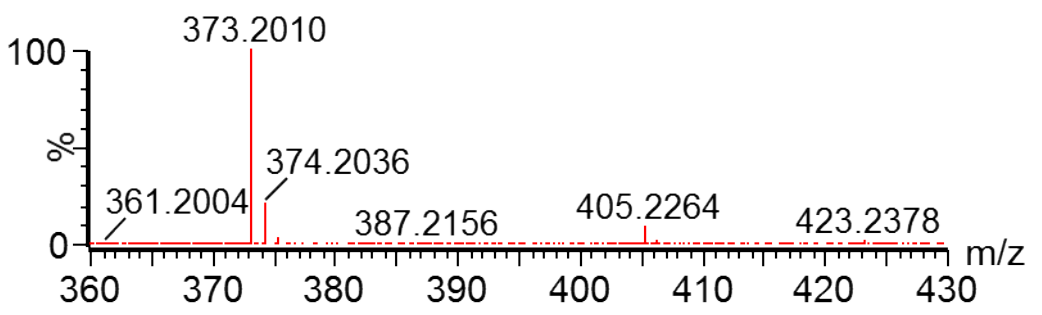

(b)

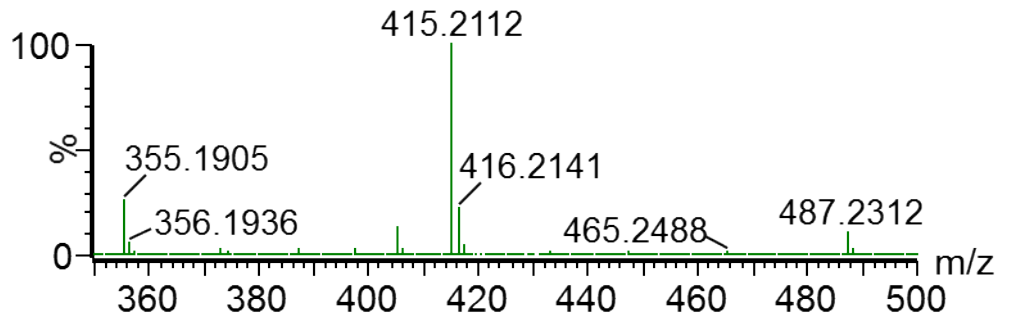

(c) $100 \quad 466.2274$

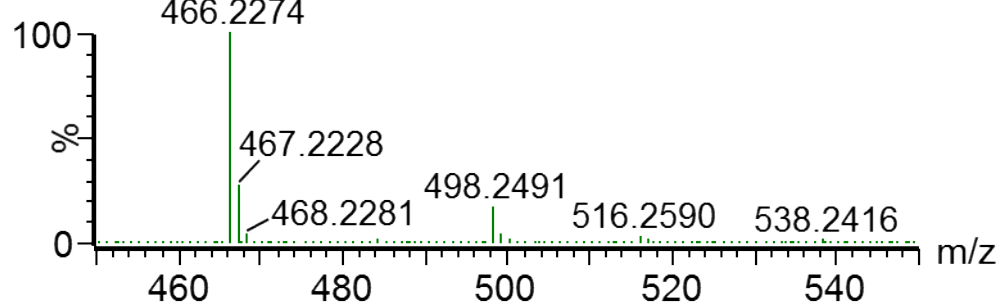

(d) $1007-508.2339$

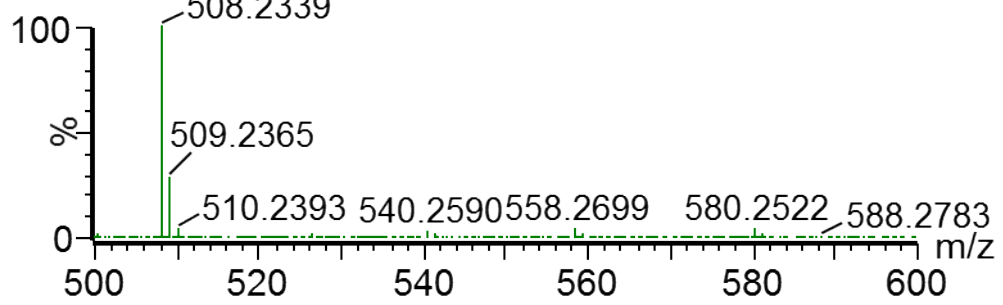

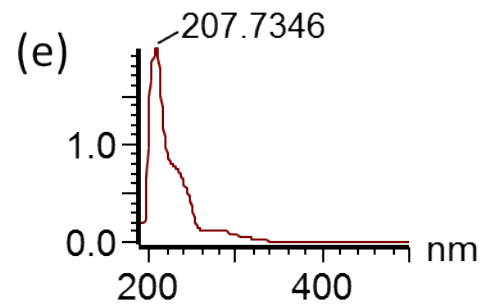

(f)

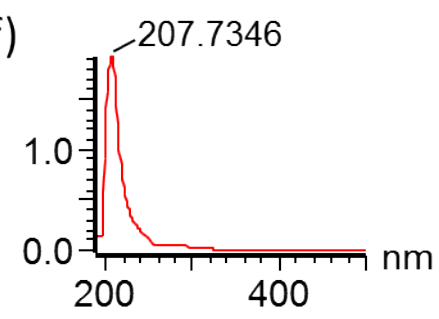

(g)

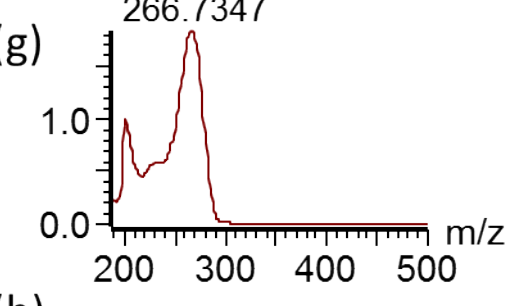

(h)

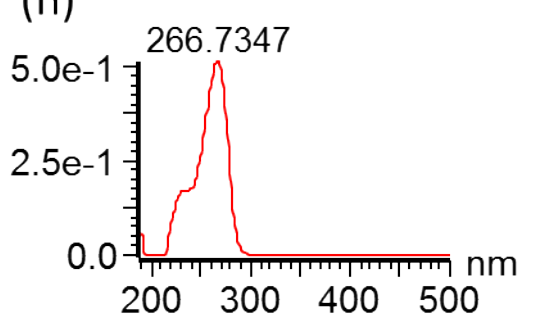


Figure S3. Different NMR spectrum of nargenicin A1 (1)

A. ${ }^{1} \mathrm{H}$ NMR spectrum of 1 in $700 \mathrm{MHz}$, DMSO-d6

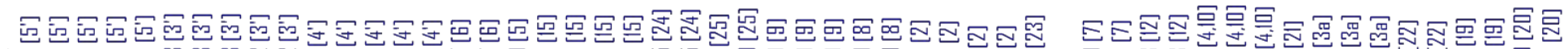

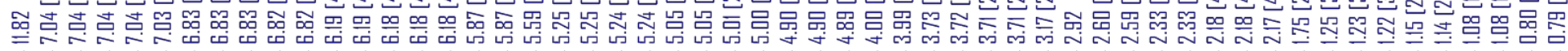

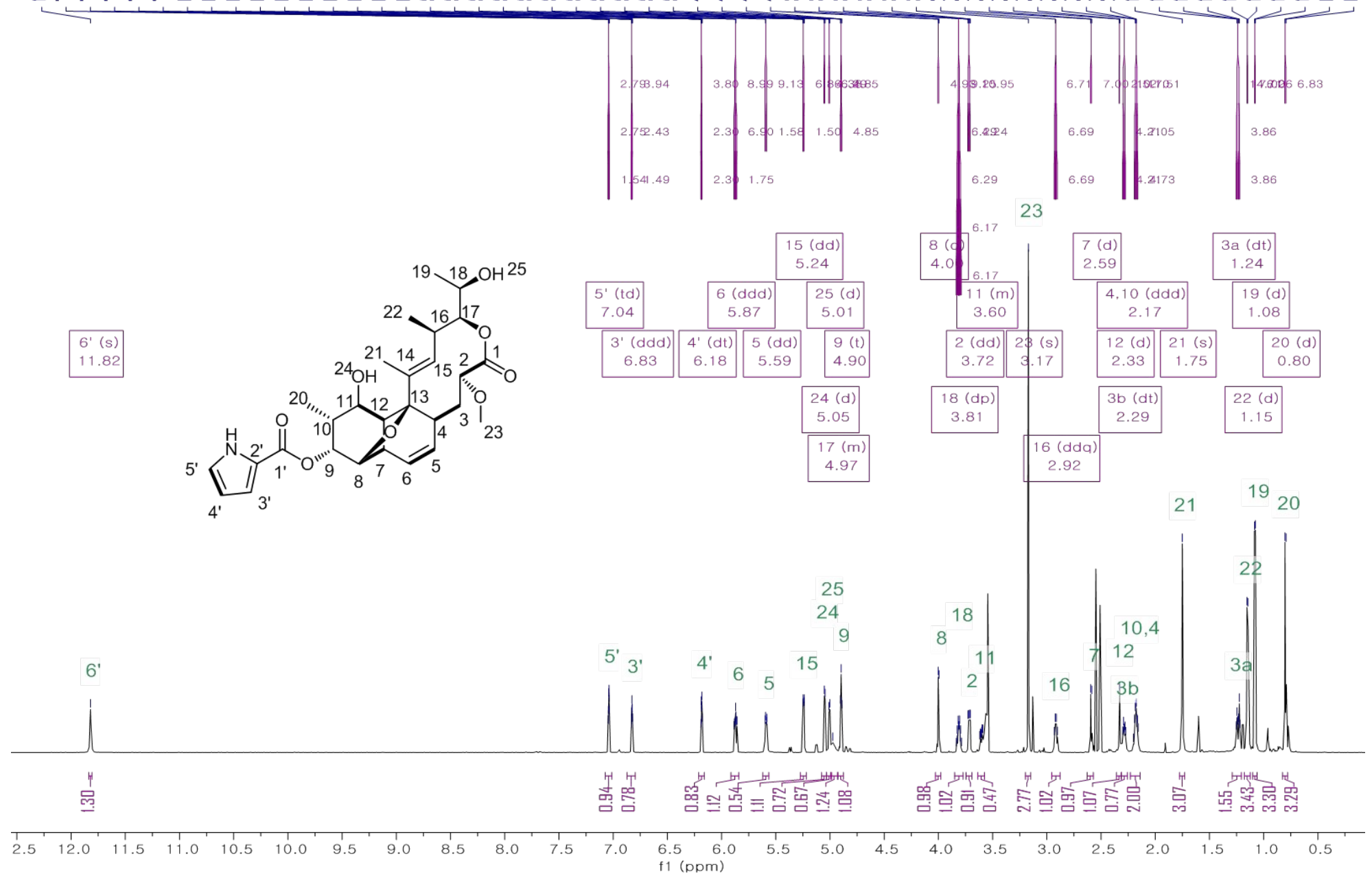


B. ${ }^{13} \mathrm{C}$ NMR spectrum of 1 in $176 \mathrm{MHz}$, DMSO-d6

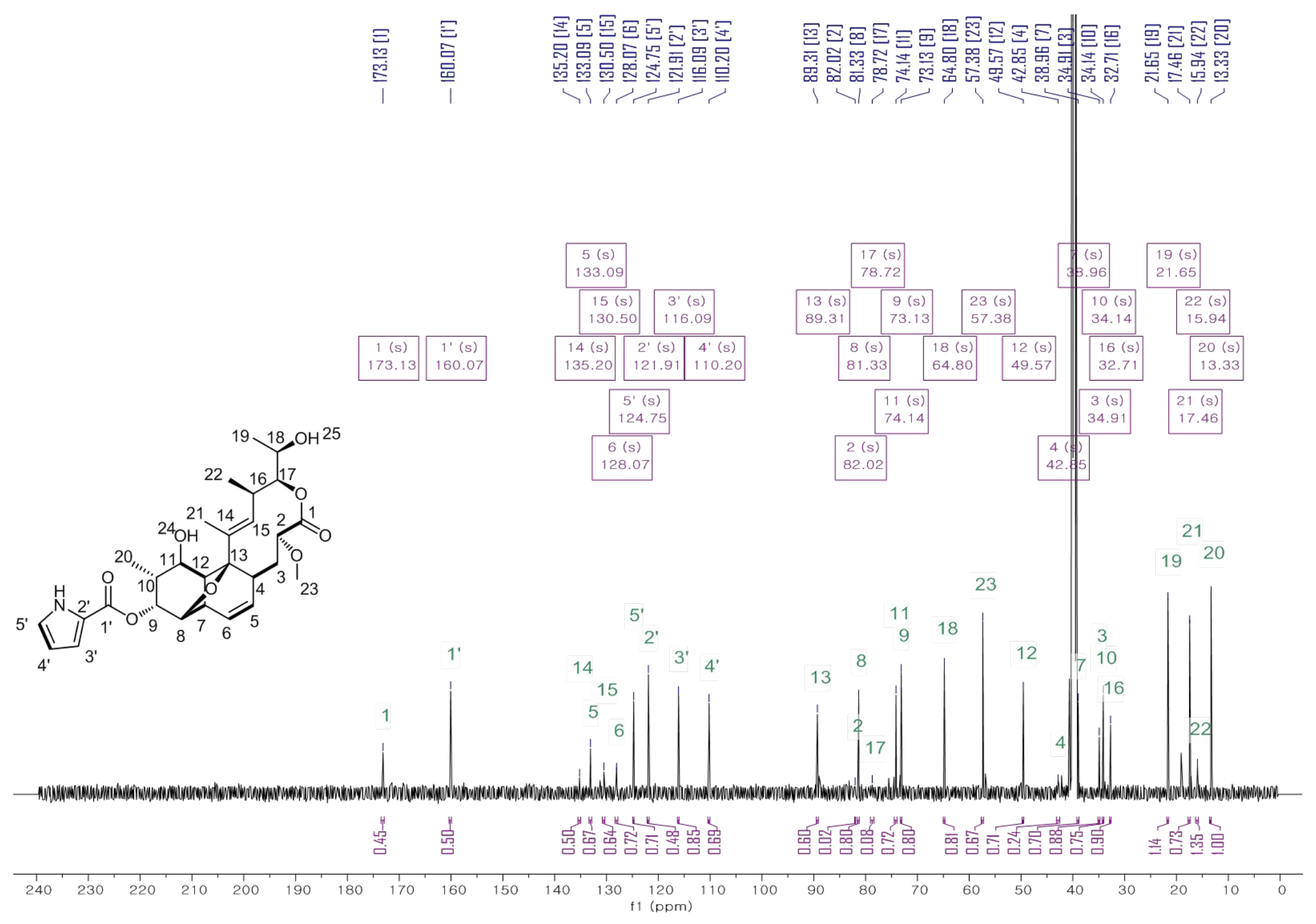


C. COSY NMR spectrum

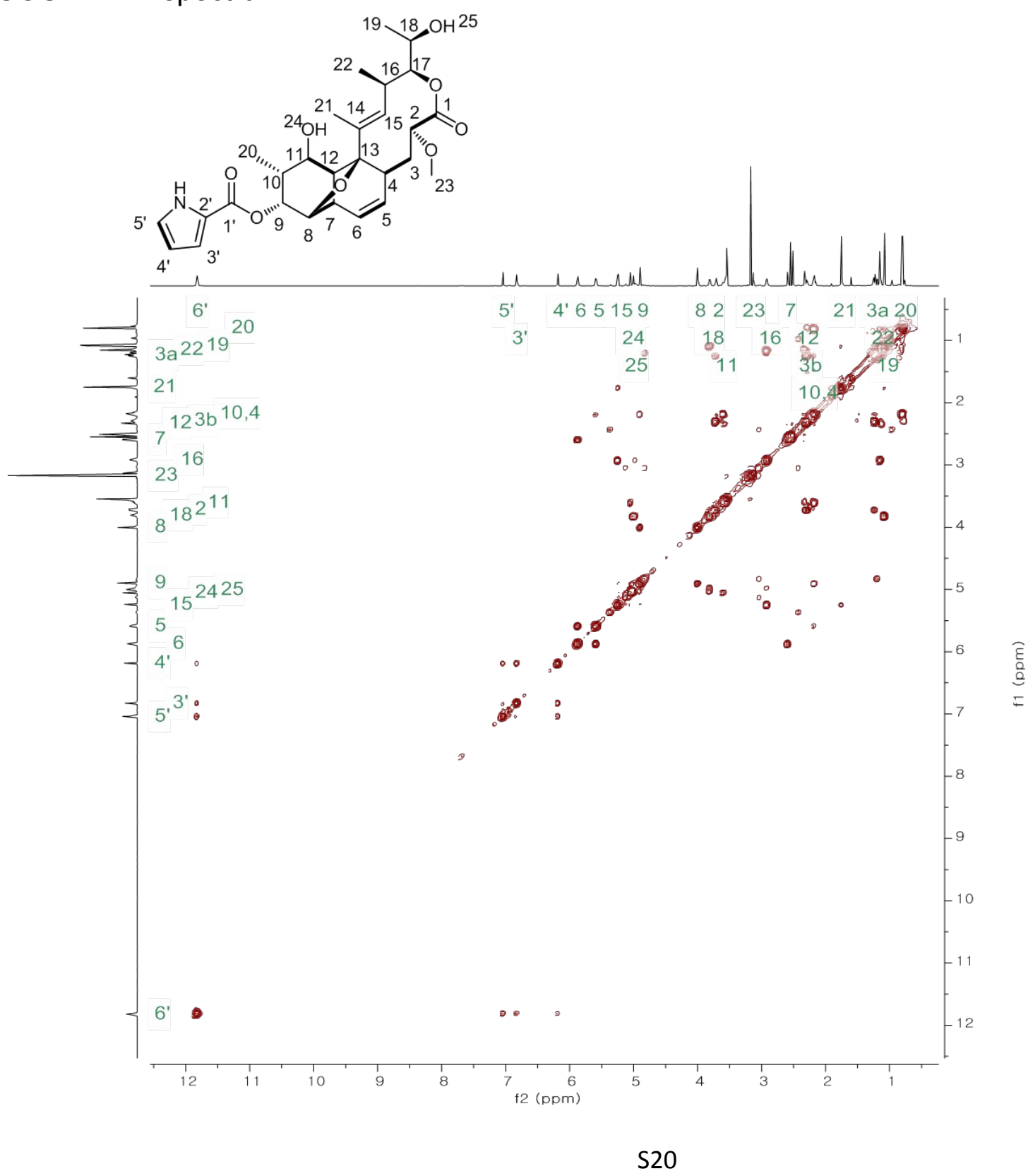


D. HSQC-DEPT NMR spectrum

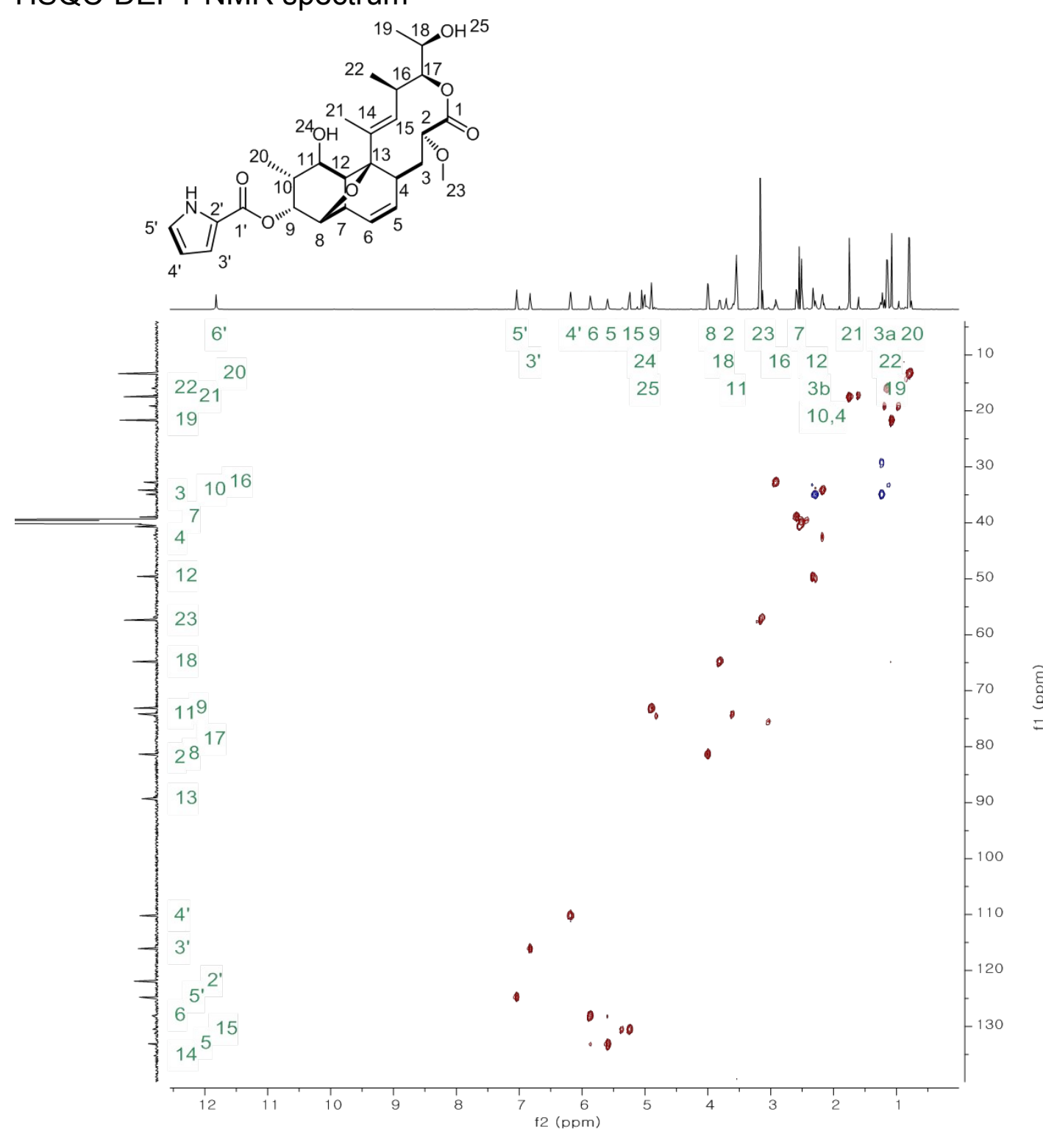


E. HMBC NMR analysis

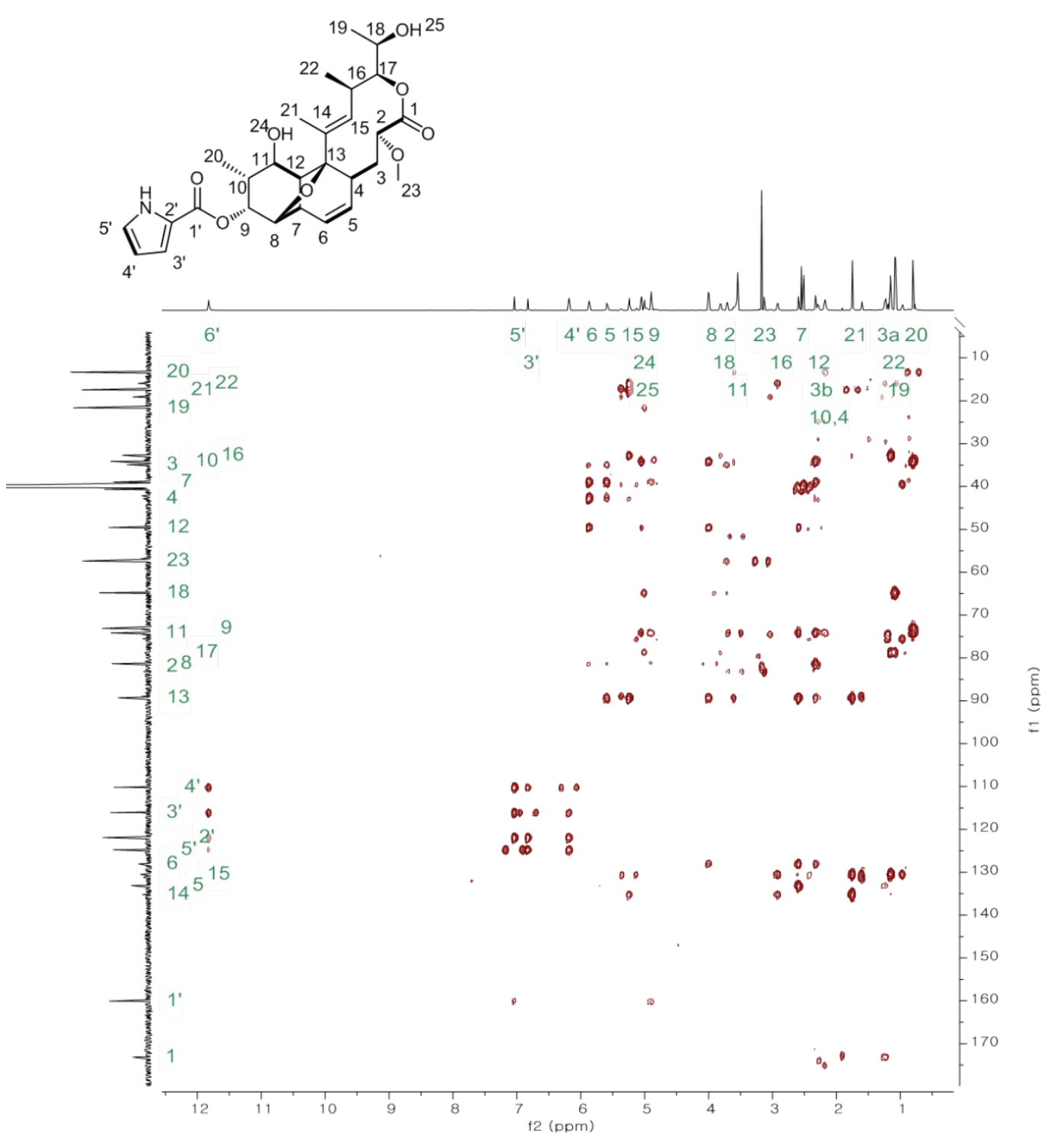


F. ROSEY NMR spectrum analysis

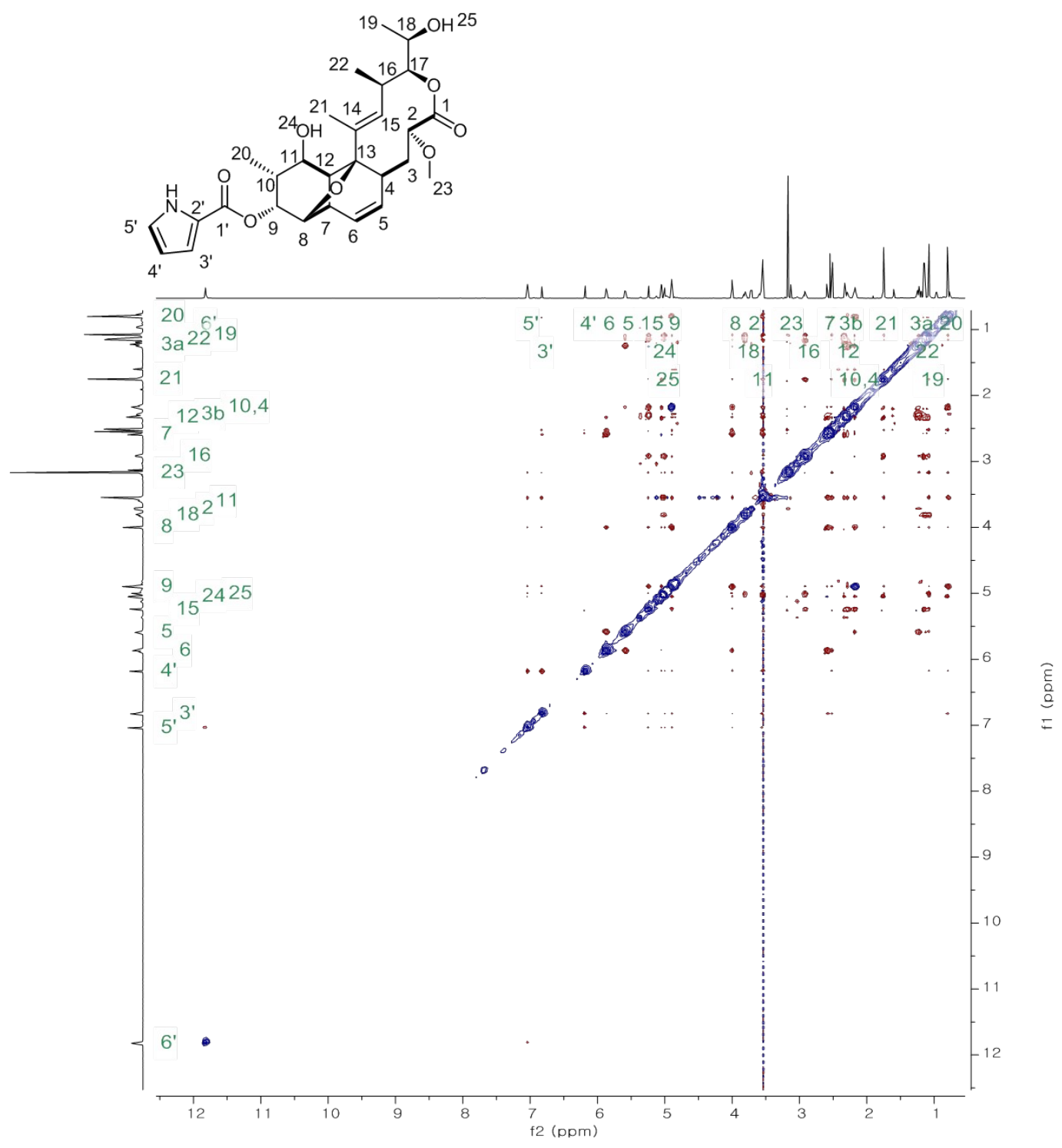


Figure S4. Diferent NMR spectrum of nodusmicin (2)

A. ${ }^{1} \mathrm{H}$ NMR spectrum of 2 in $700 \mathrm{MHz}$,DMSO-d6.

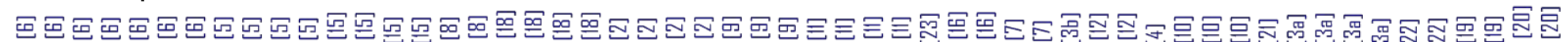

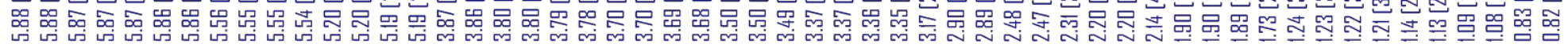

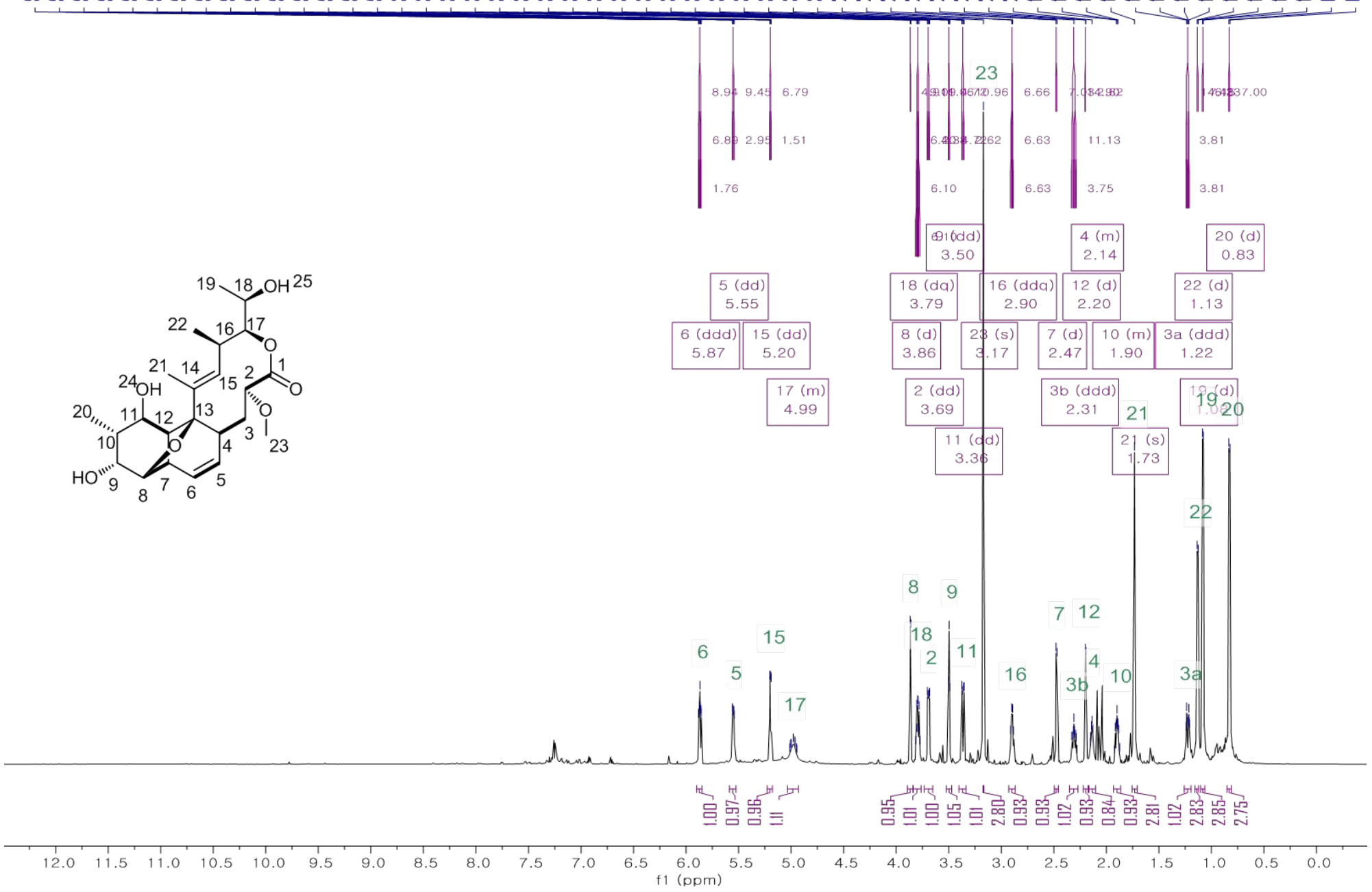


B. ${ }^{1} \mathrm{H}$ NMR spectrum of 2 in $700 \mathrm{MHz}$,DMSO-d6 (zoomed view $0.6 \mathrm{ppm}$ to $6 \mathrm{ppm}$ )

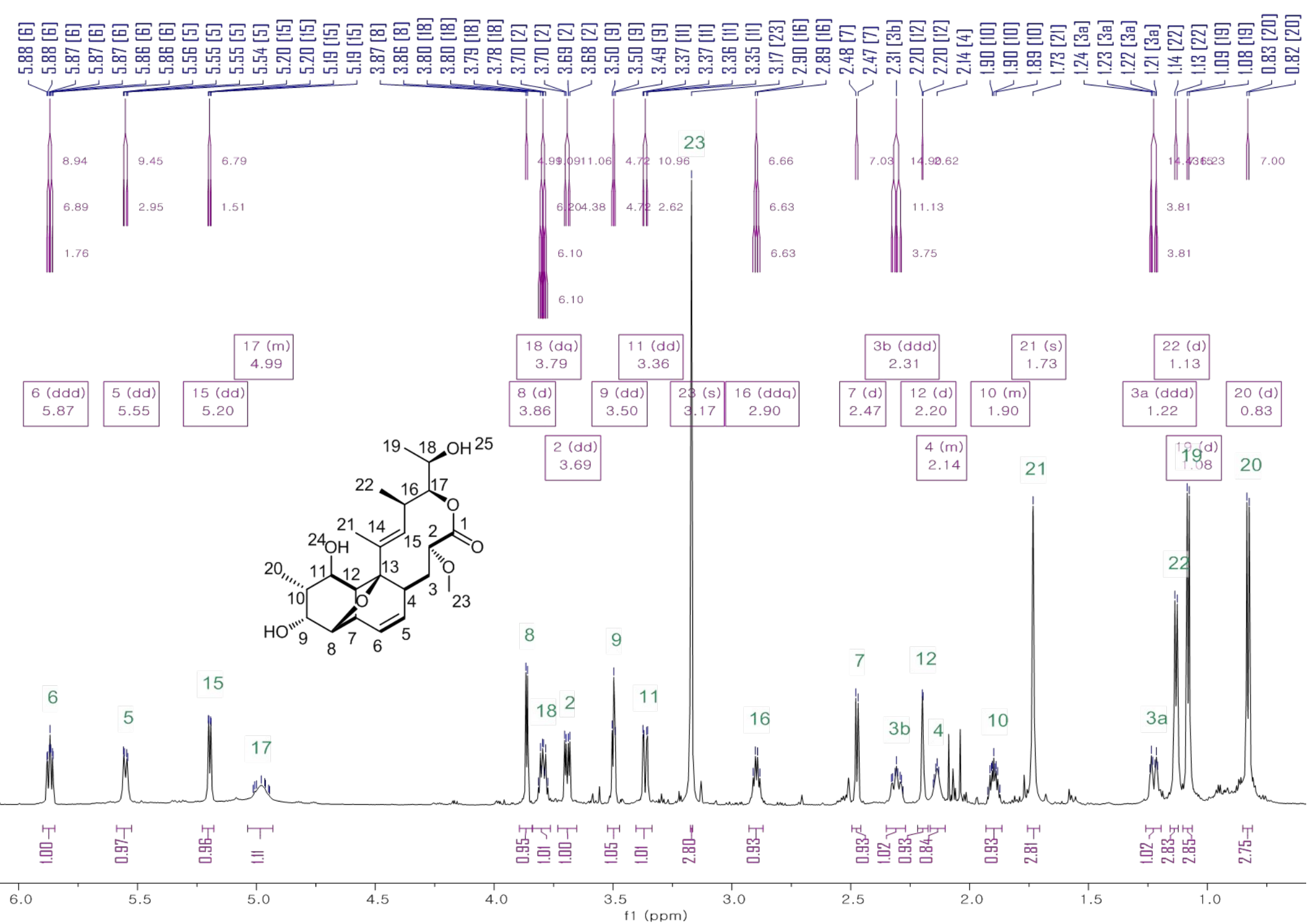


C. ${ }^{13} \mathrm{C}$ NMR spectrum of 2 in $176 \mathrm{MHz}$, DMSO-d6
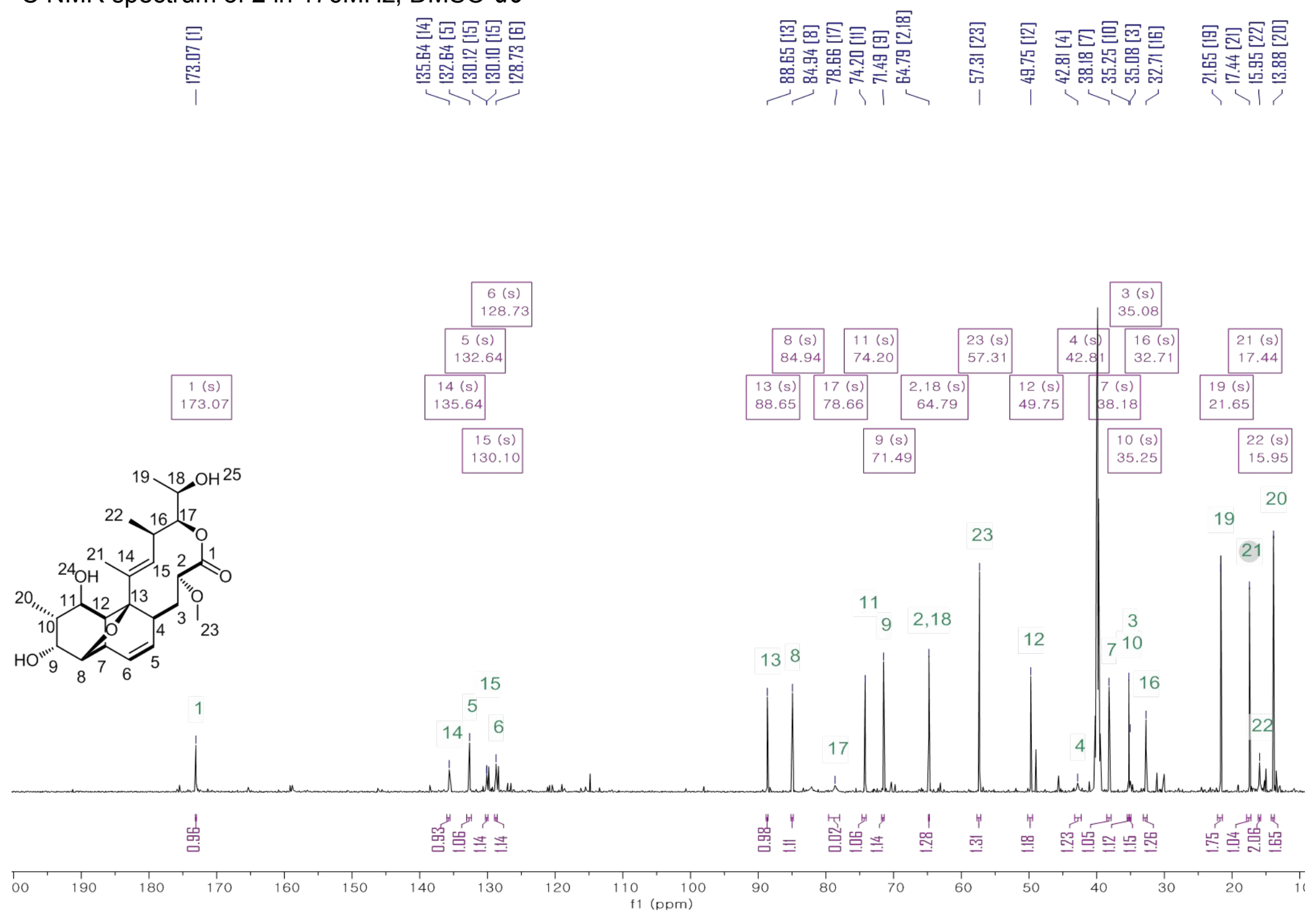
${ }^{13} \mathrm{C}$ NMR spectrum of 2 in $176 \mathrm{MHz}$, DMSO-d6 (refined view)

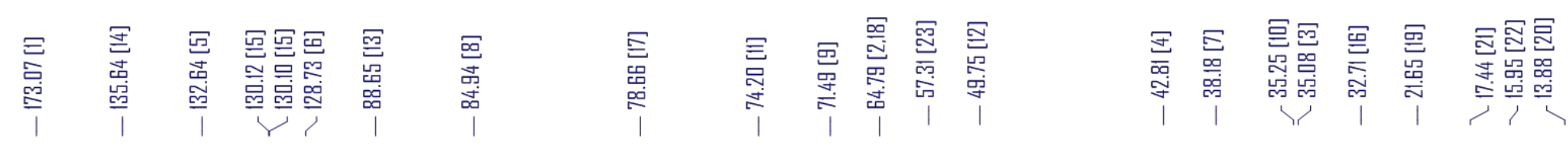
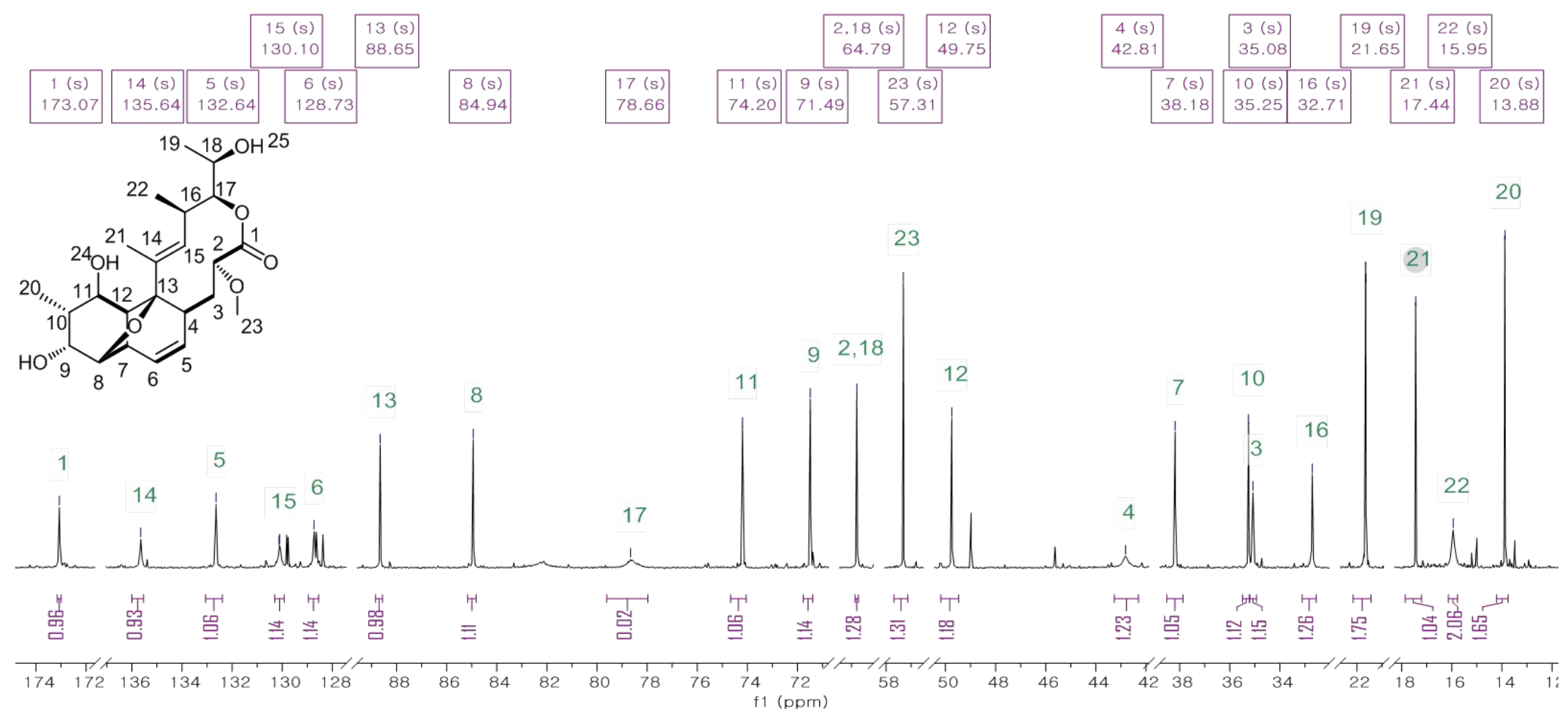
D. COSY NMR spectrum

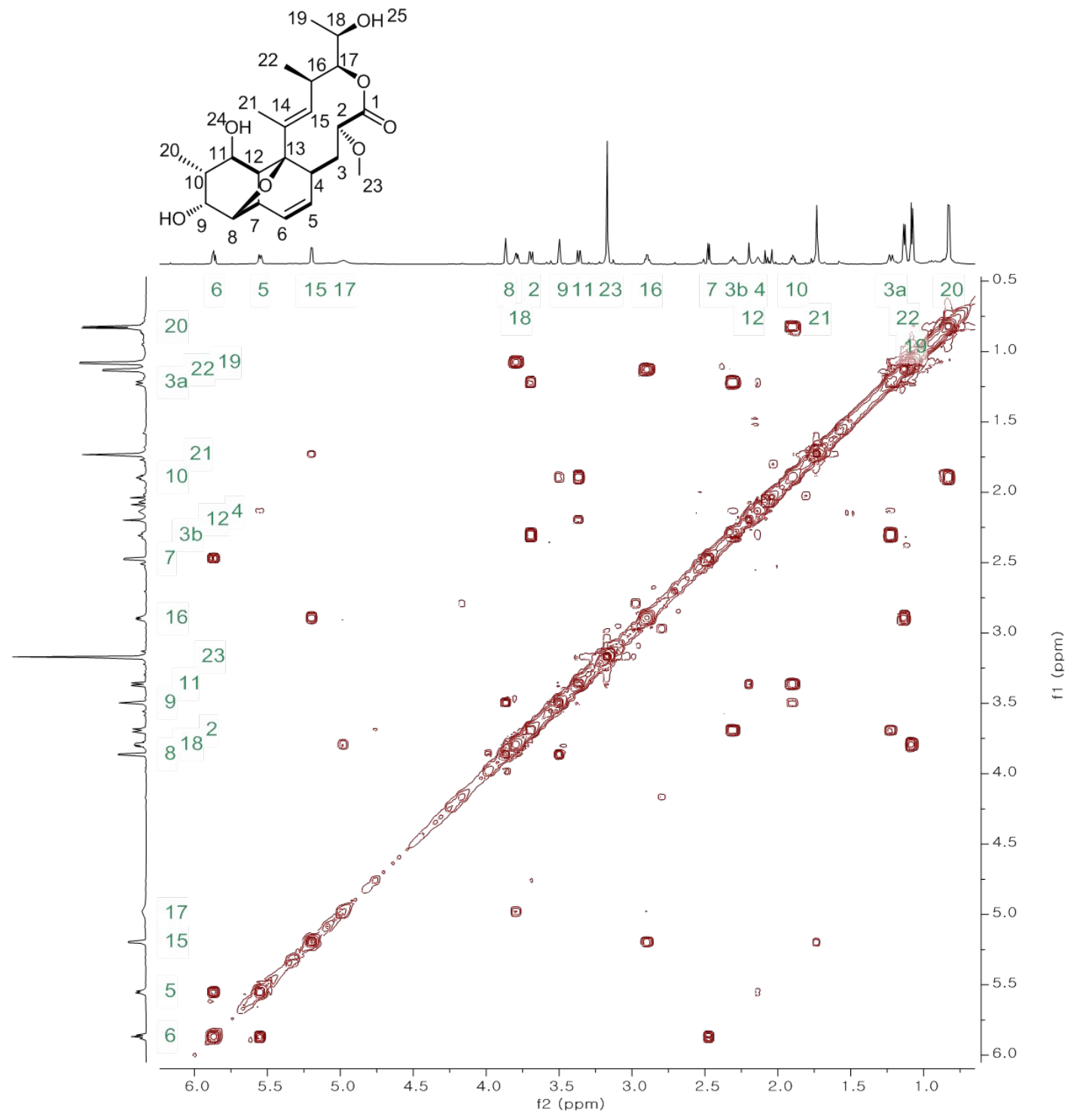


E. HSQC NMR spectrum

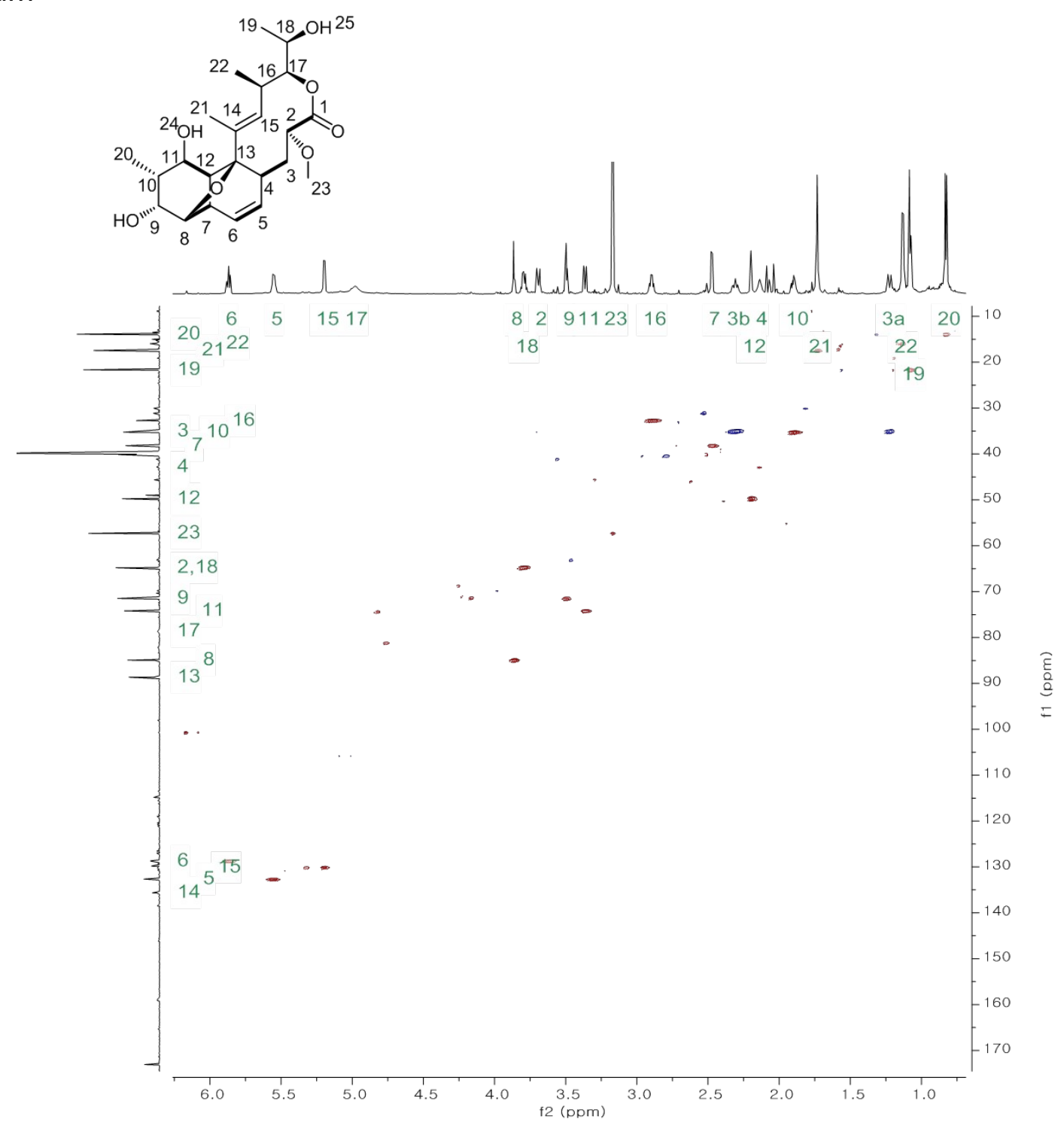




\section{F. HSQC NMR spectrum (refined view)}

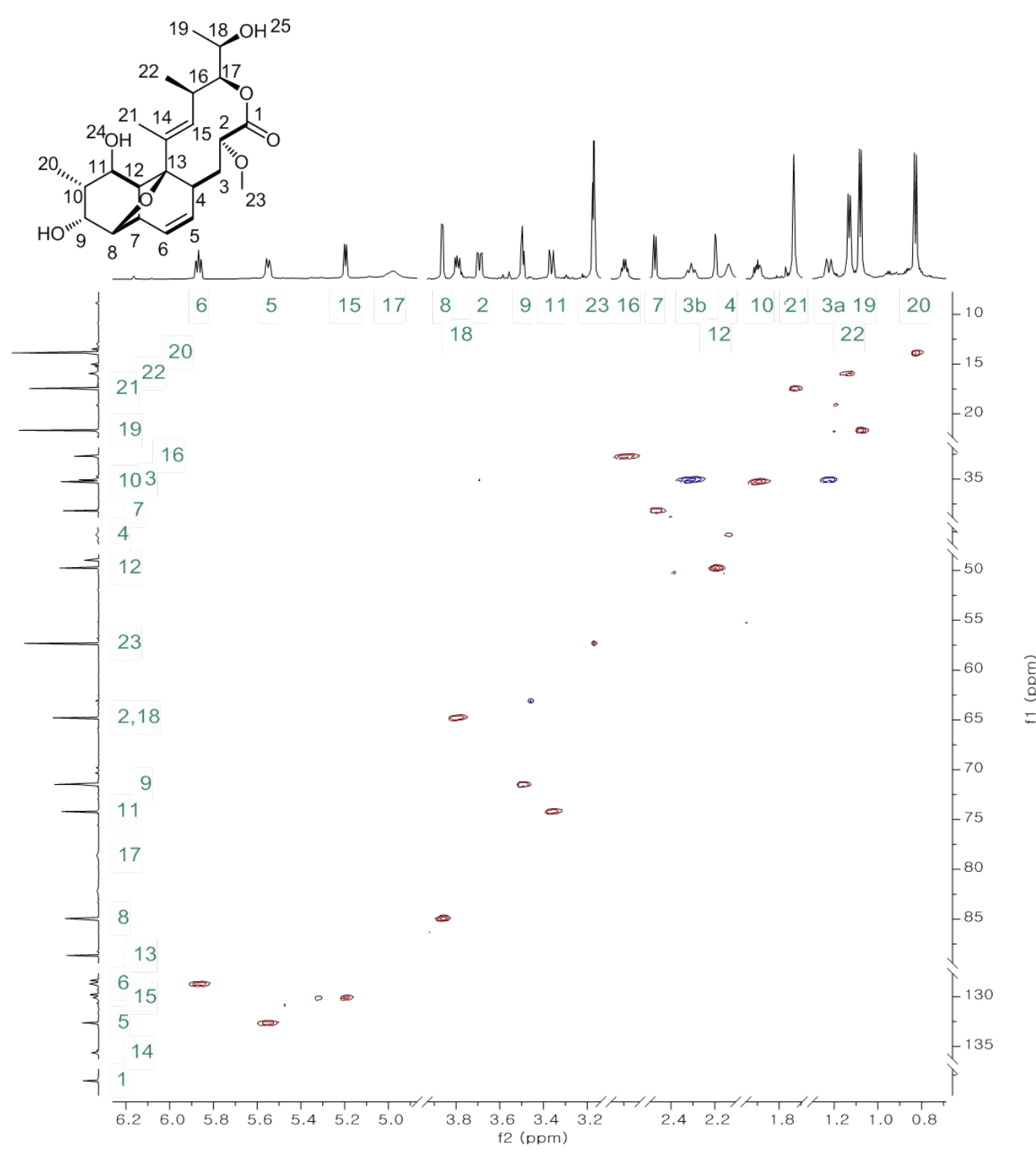


G. HMBC NMR spectrum

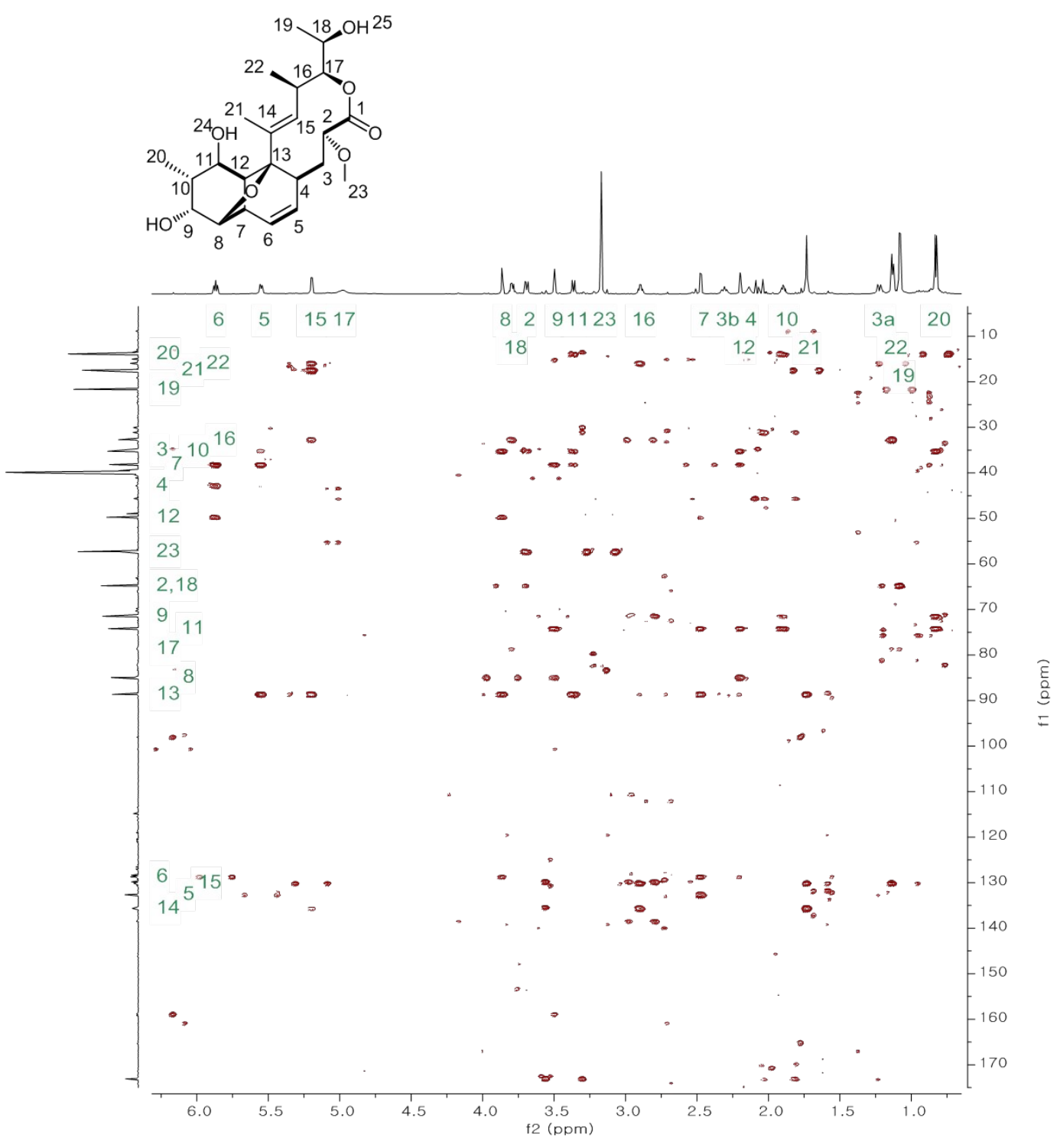


H. HMBC NMR spectrum (refined view)

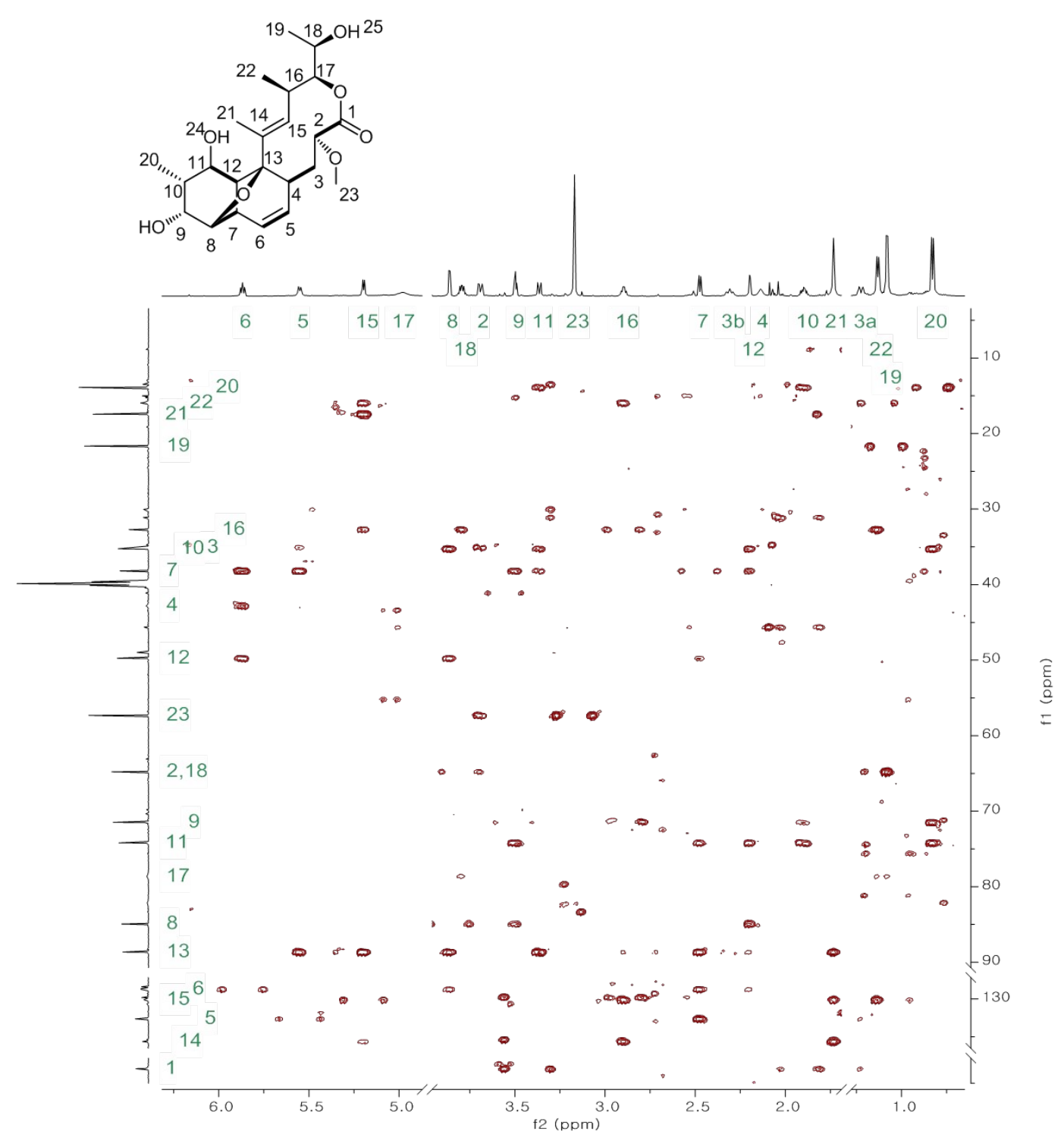


I. ROESY NMR spectrum

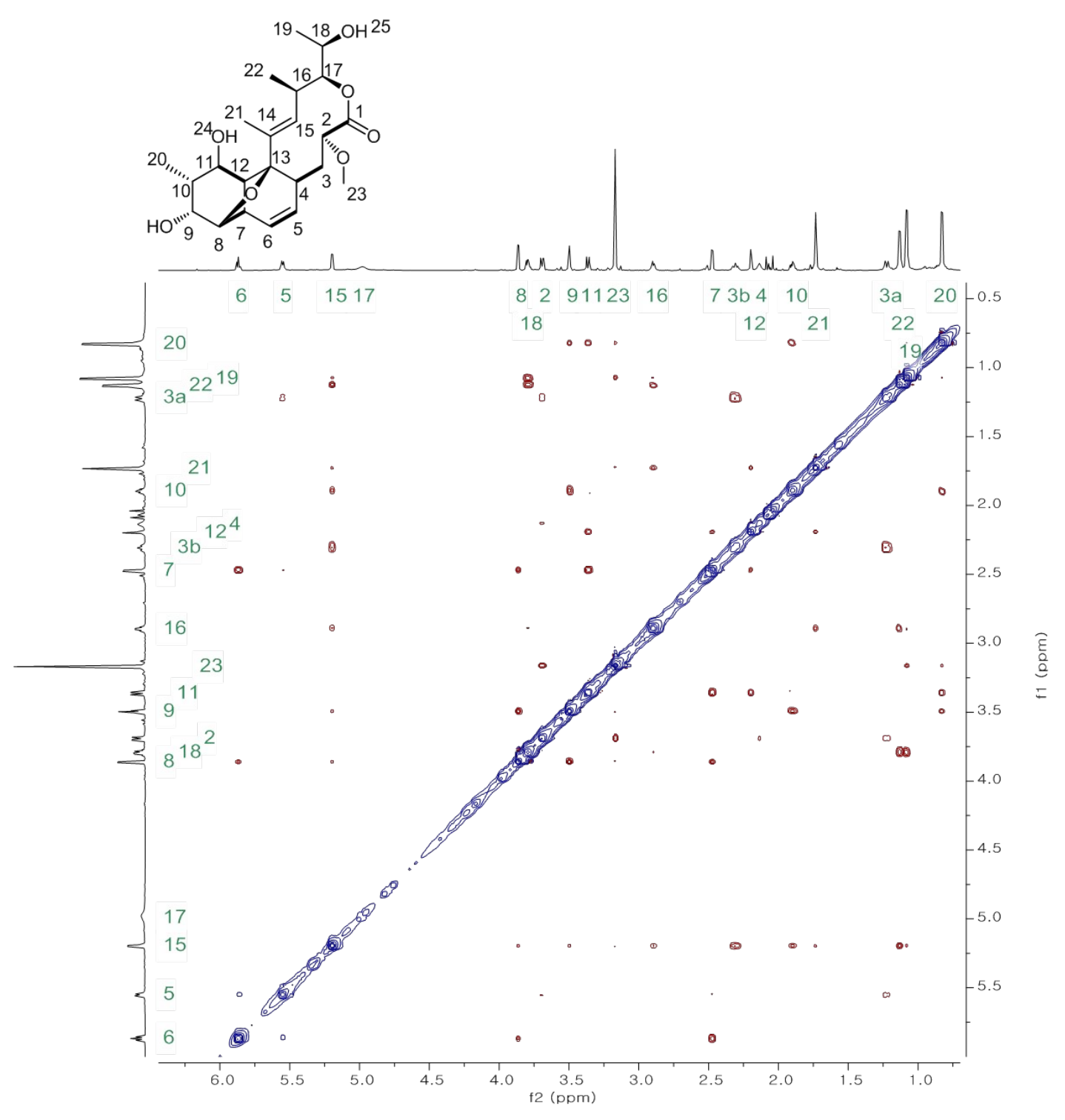


Figure S5. Different NMR spectrum of 18-O-acetyl nargenicin (13)

A. ${ }^{1} \mathrm{H}$ NMR spectrum of 13 in $700 \mathrm{MHz}$, DMSO-d6

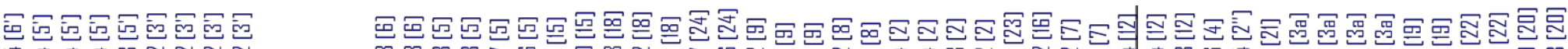

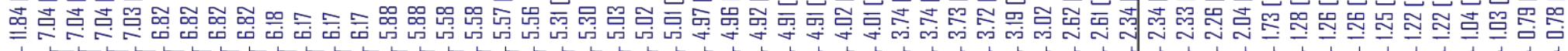

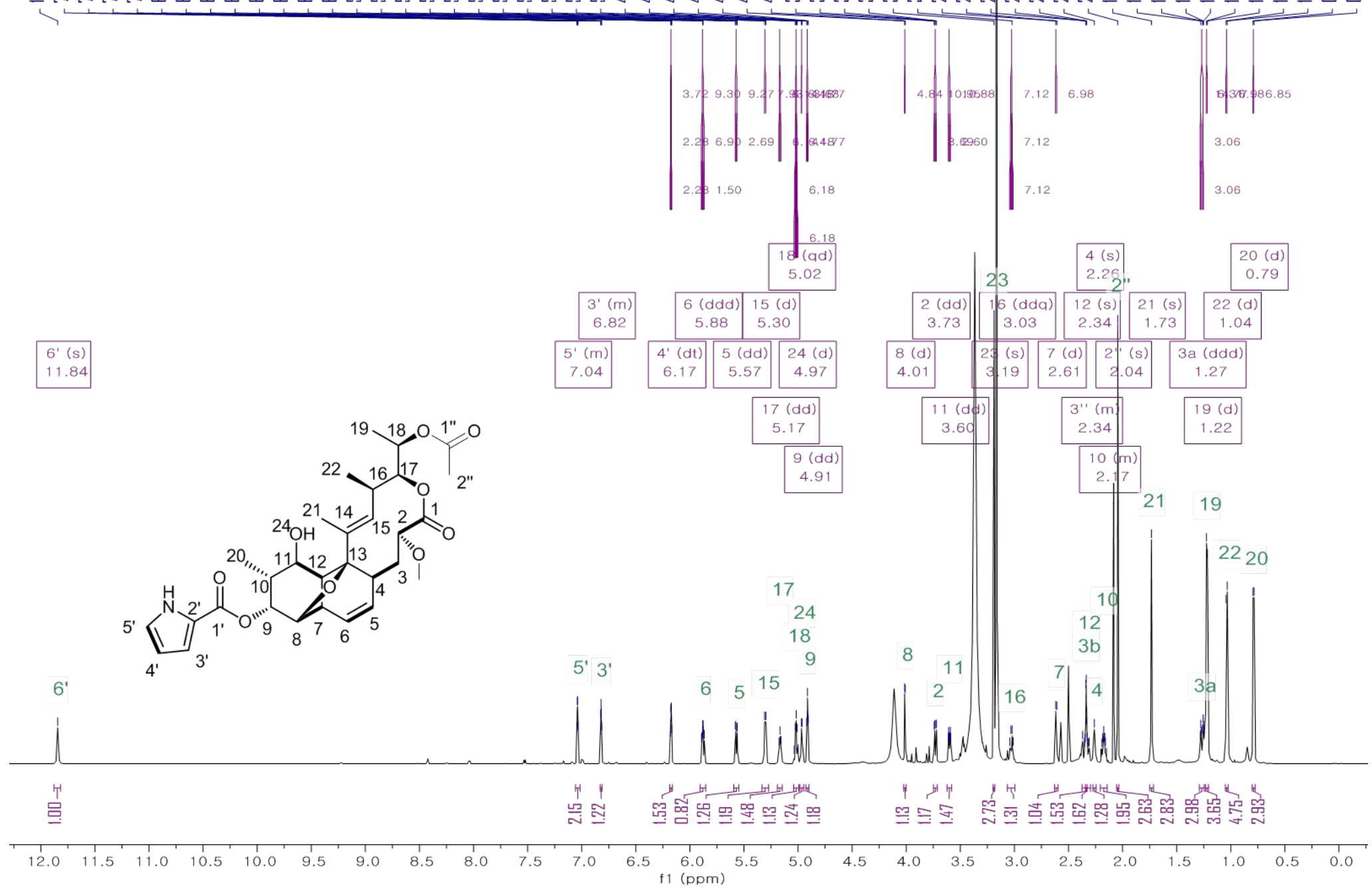


B. ${ }^{1} \mathrm{H}$ NMR spectrum of 13 in $700 \mathrm{MHz}$, DMSO-d6 (refined view)

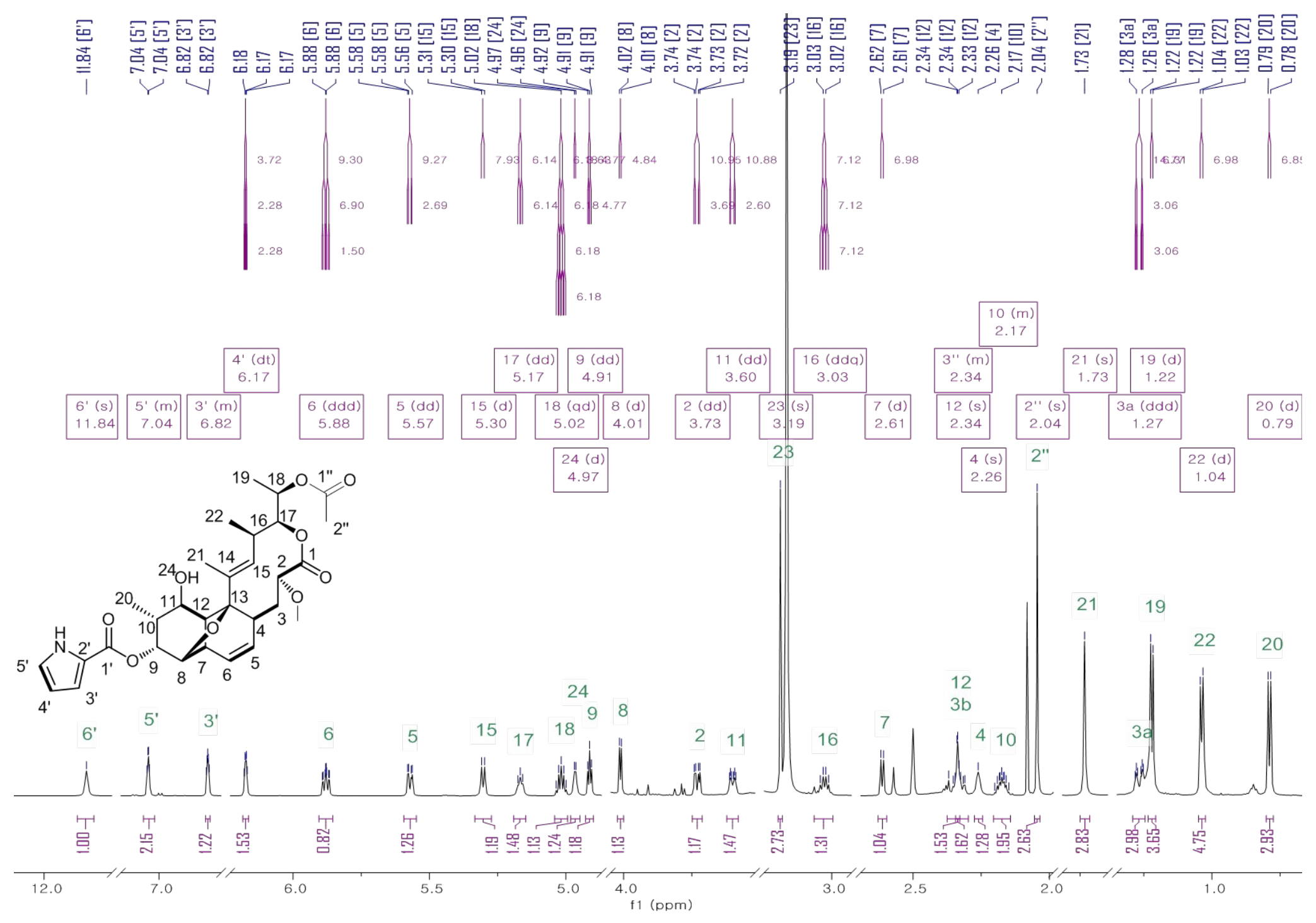


C. ${ }^{13} \mathrm{C}$ NMR spectrum of 13 in $176 \mathrm{MHz}$, DMSO-d6

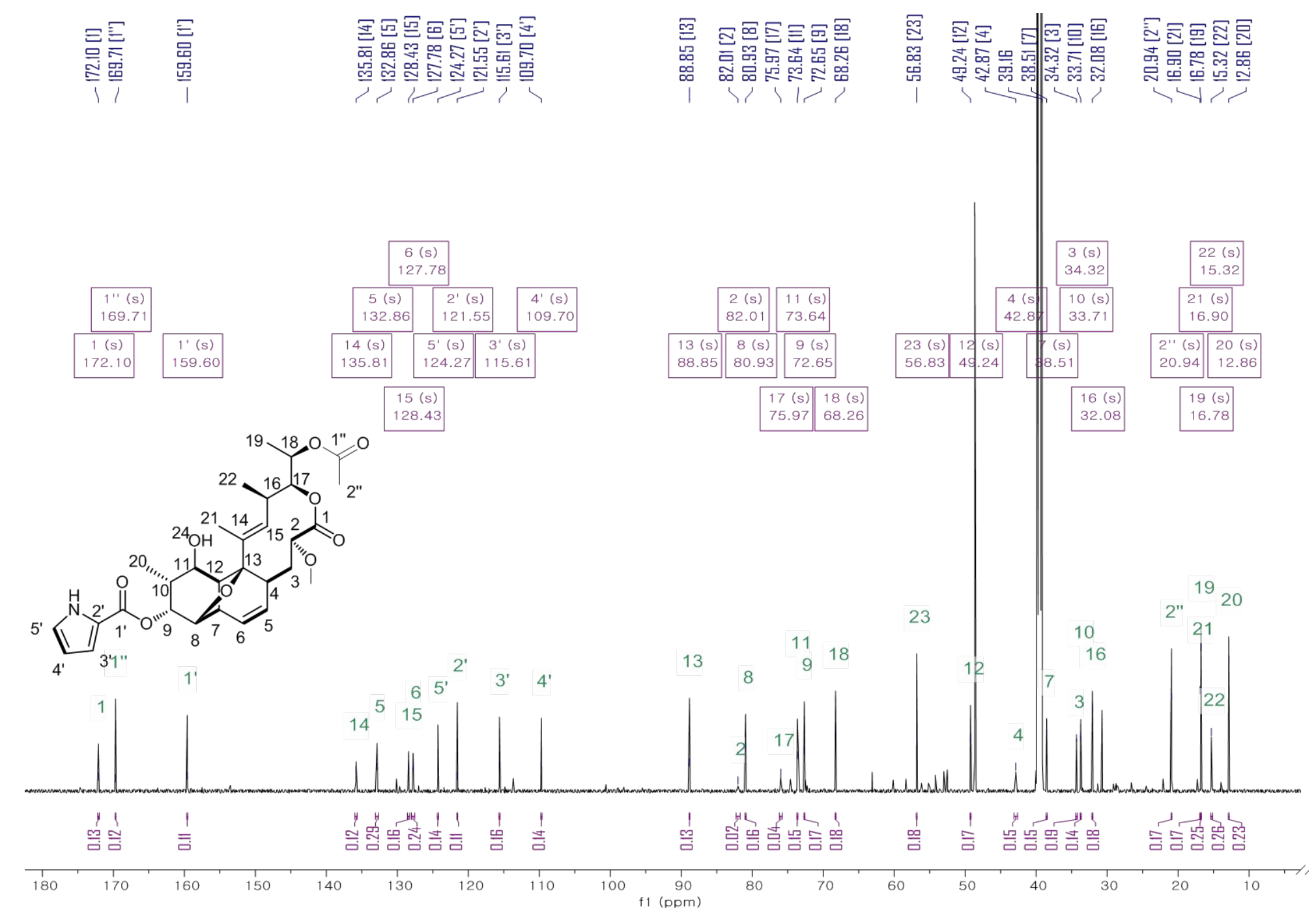


D. ${ }^{13} \mathrm{C}$ NMR spectrum of 13 in $176 \mathrm{MHz}$, DMSO-d6 (refined view)

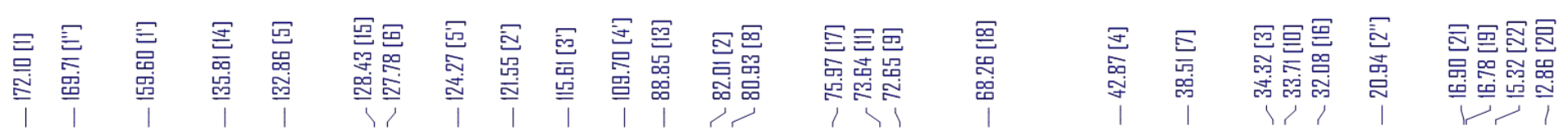

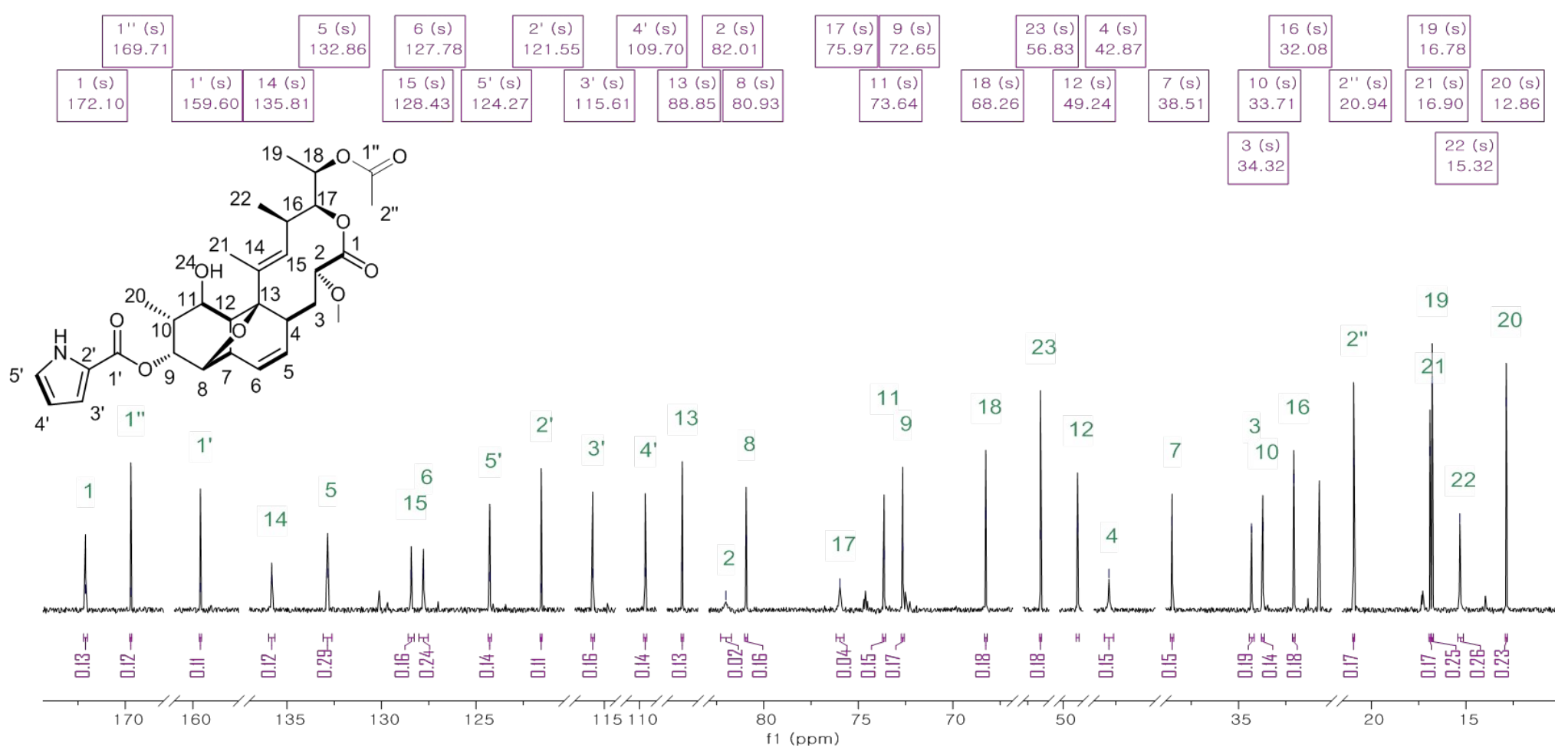


E. COSY NMR spectrum

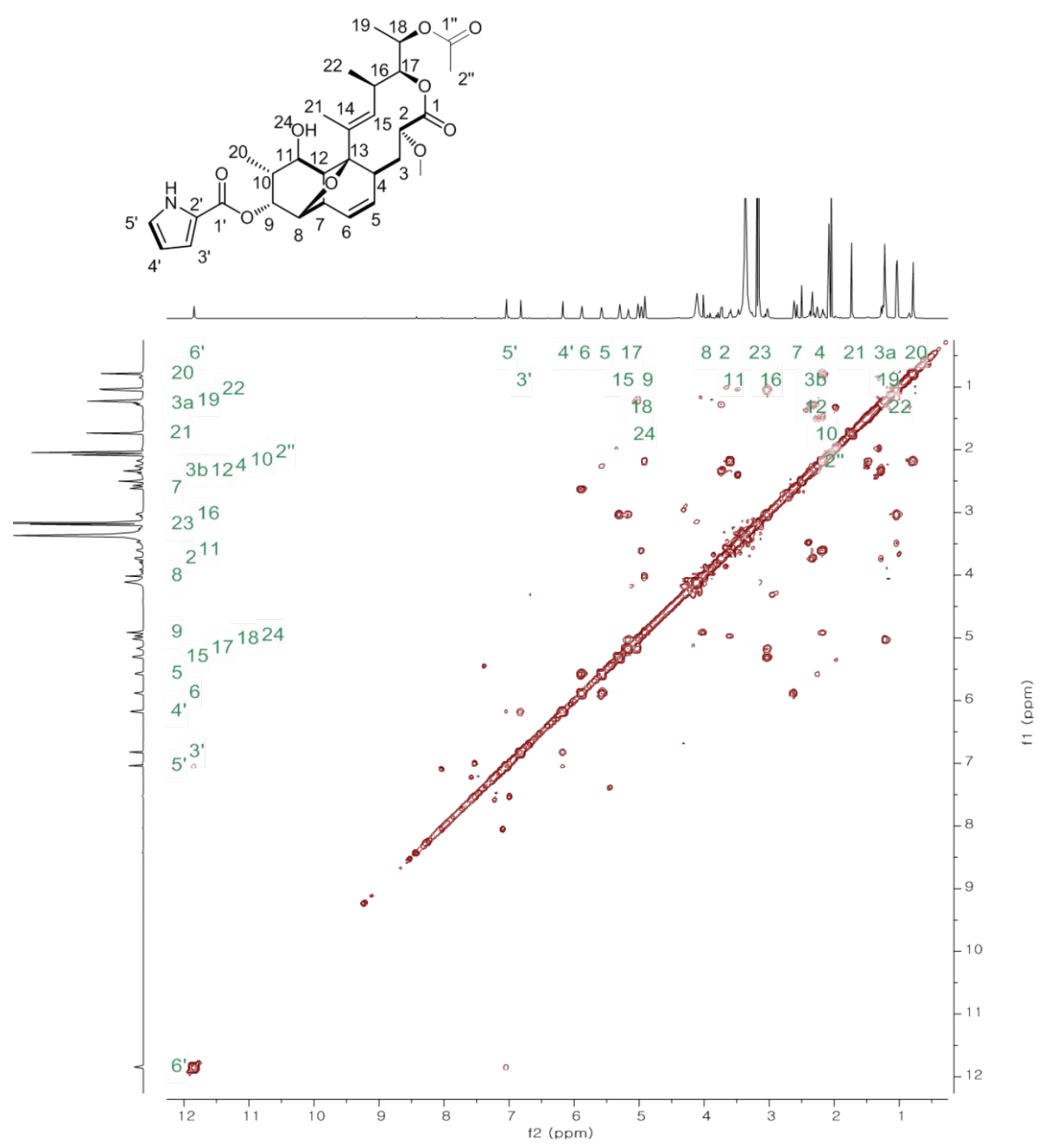


F. COSY NMR spectrum (refined view)

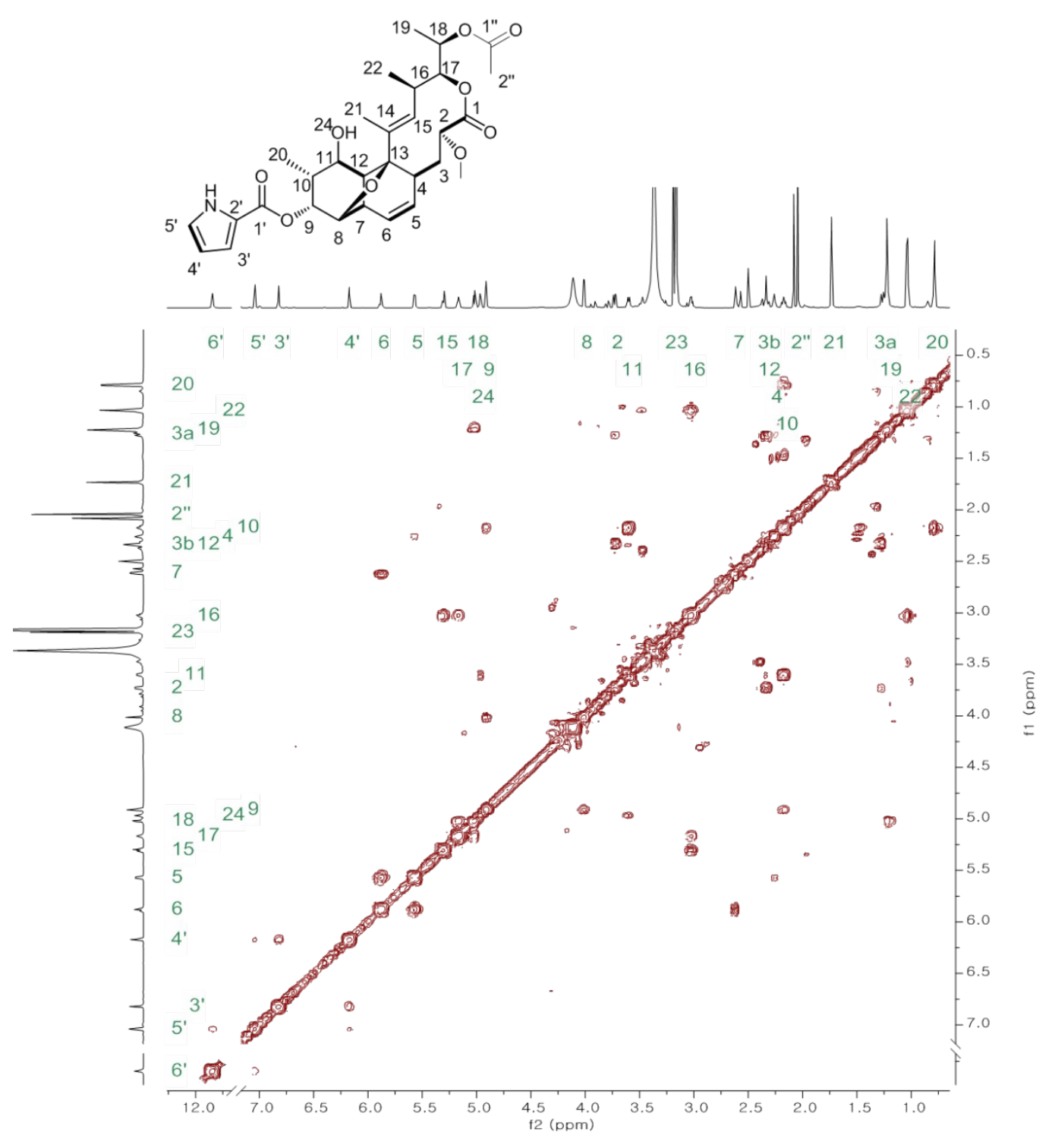


G. HSQC-DEPT NMR spectrum

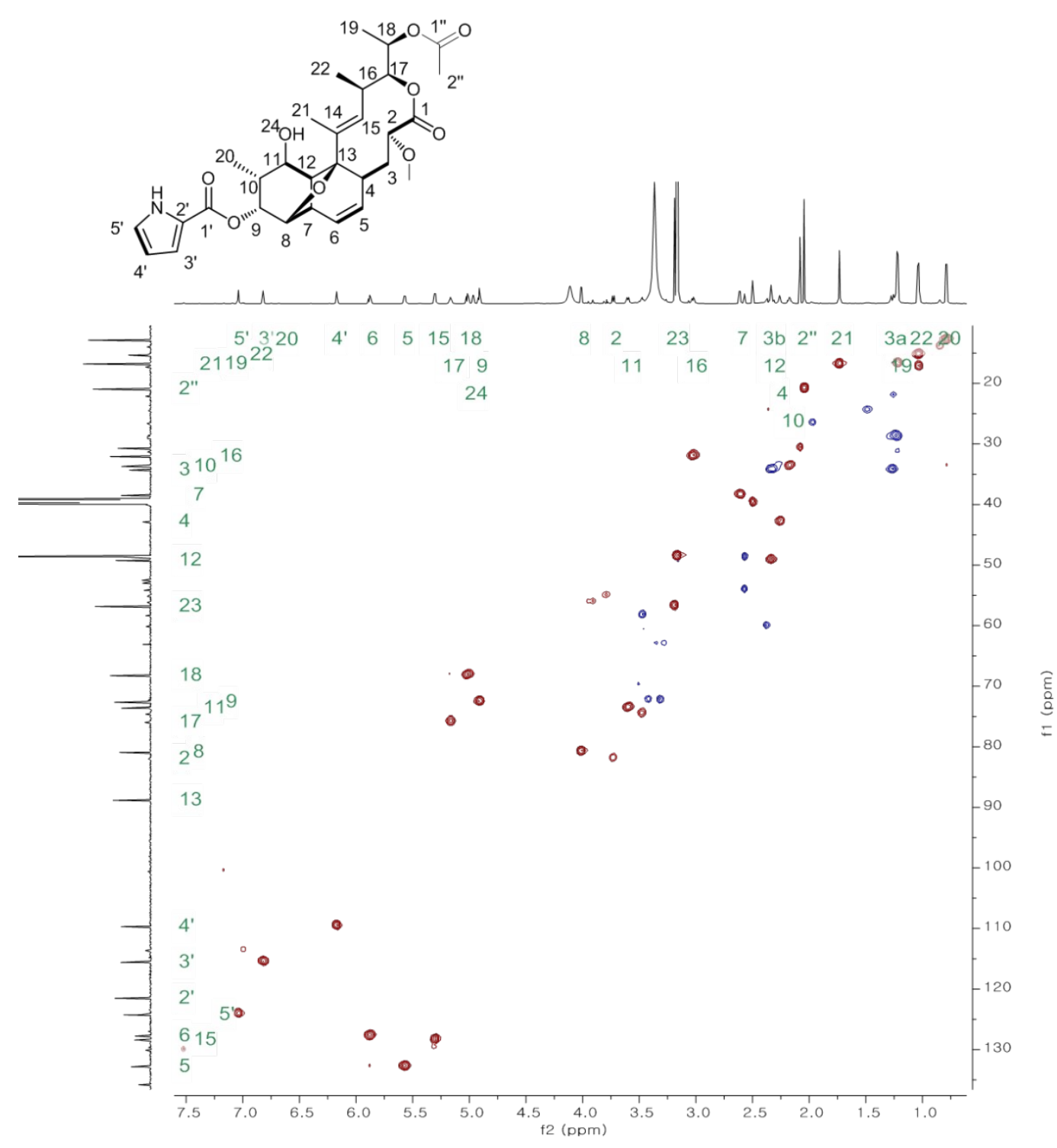


H. HSQC NMR spectrum (refined view)

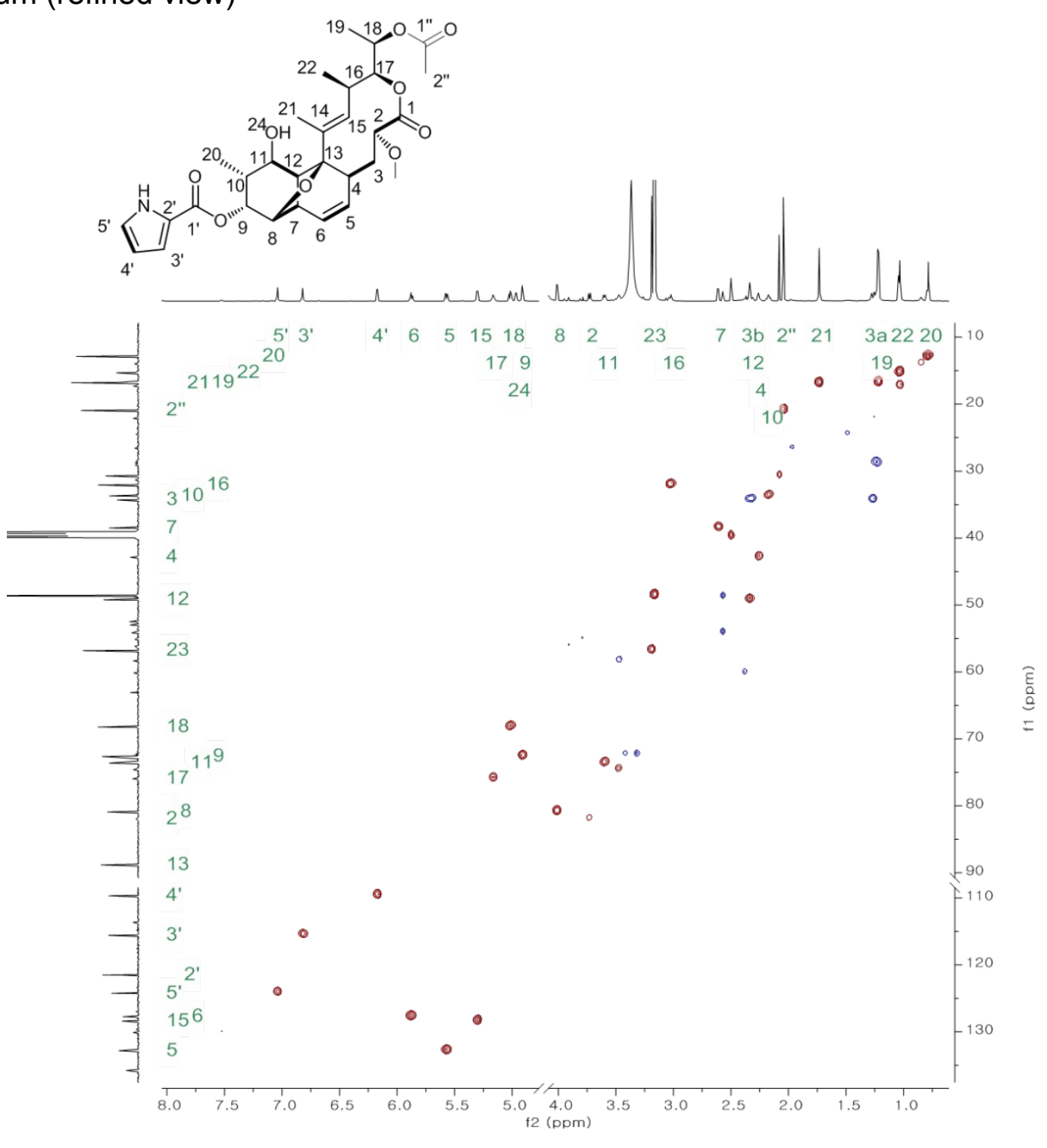


I. HMBC NMR spectrum

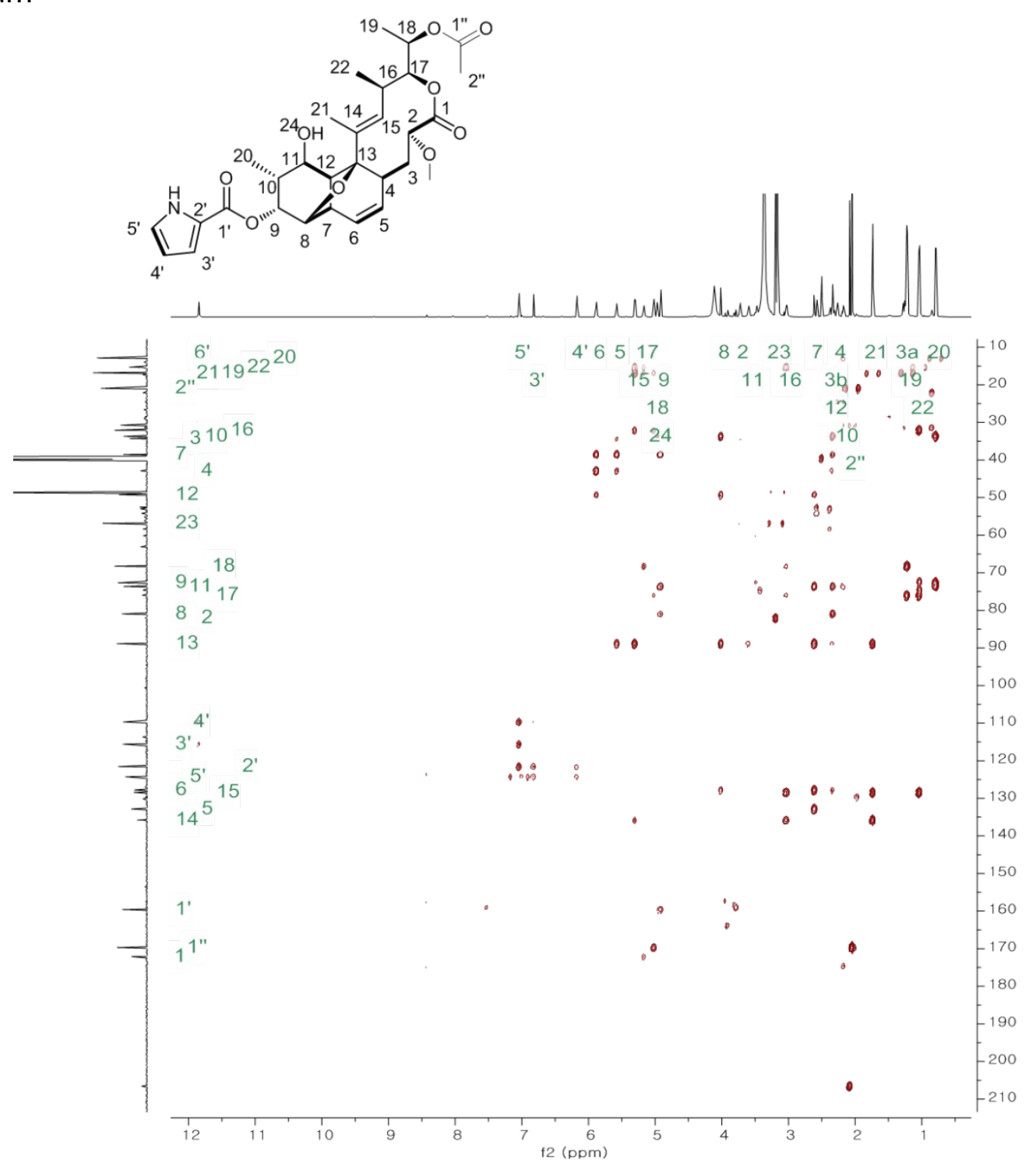


J. HMBC NMR spectrum (refined view)

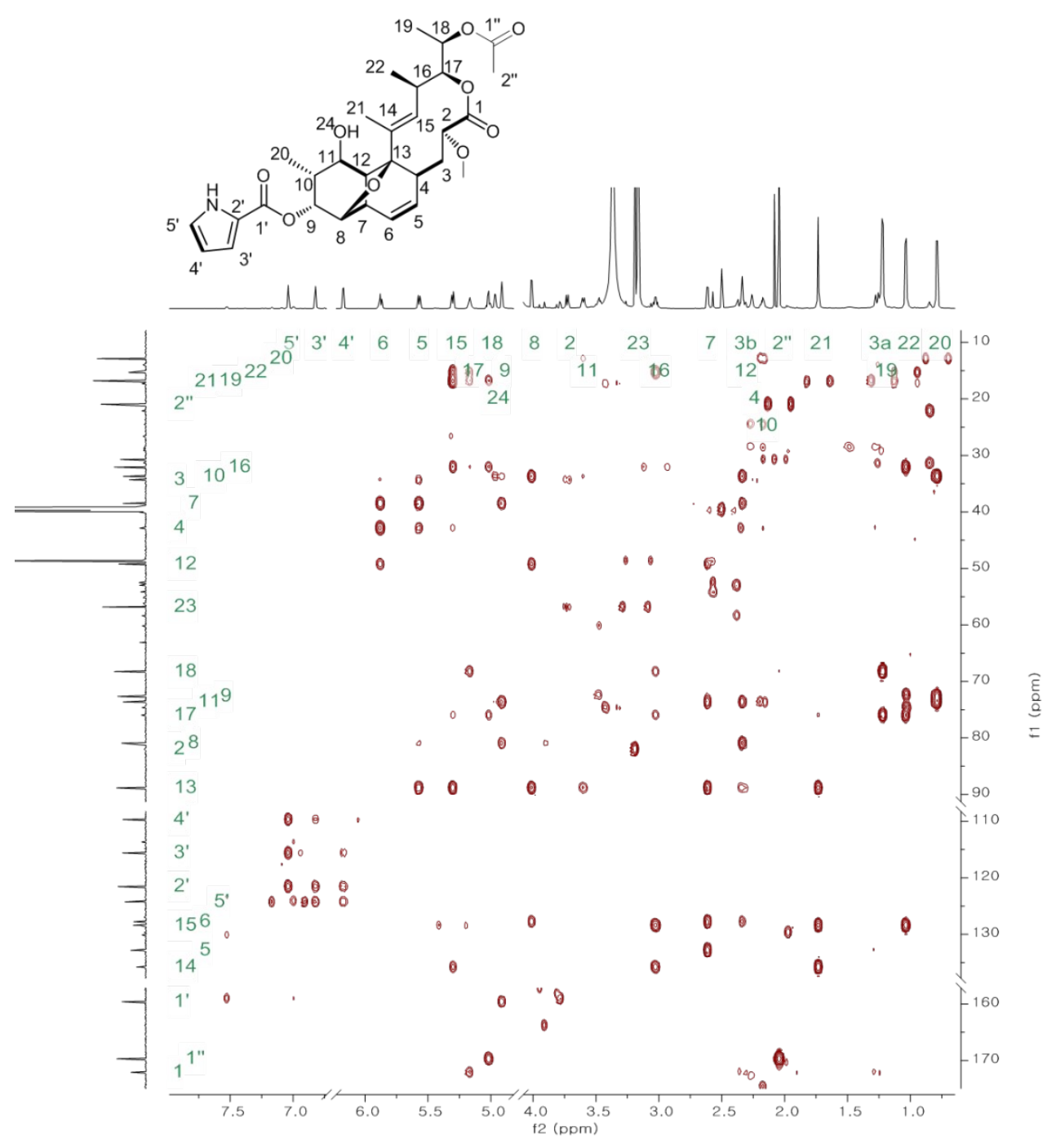


K. ROSEY NMR spectrum

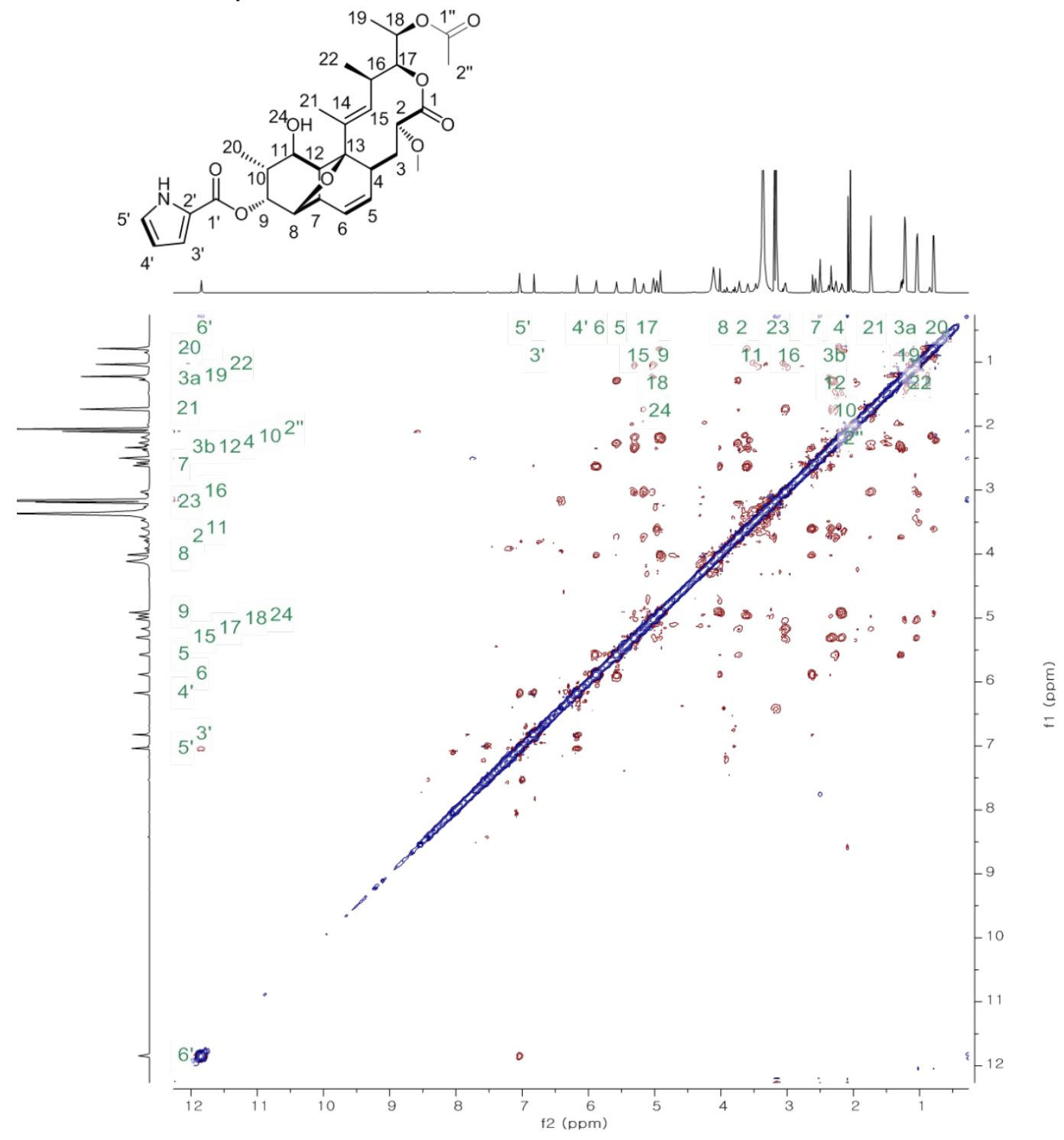


L. ROSEY NMR spectrum (refined view)

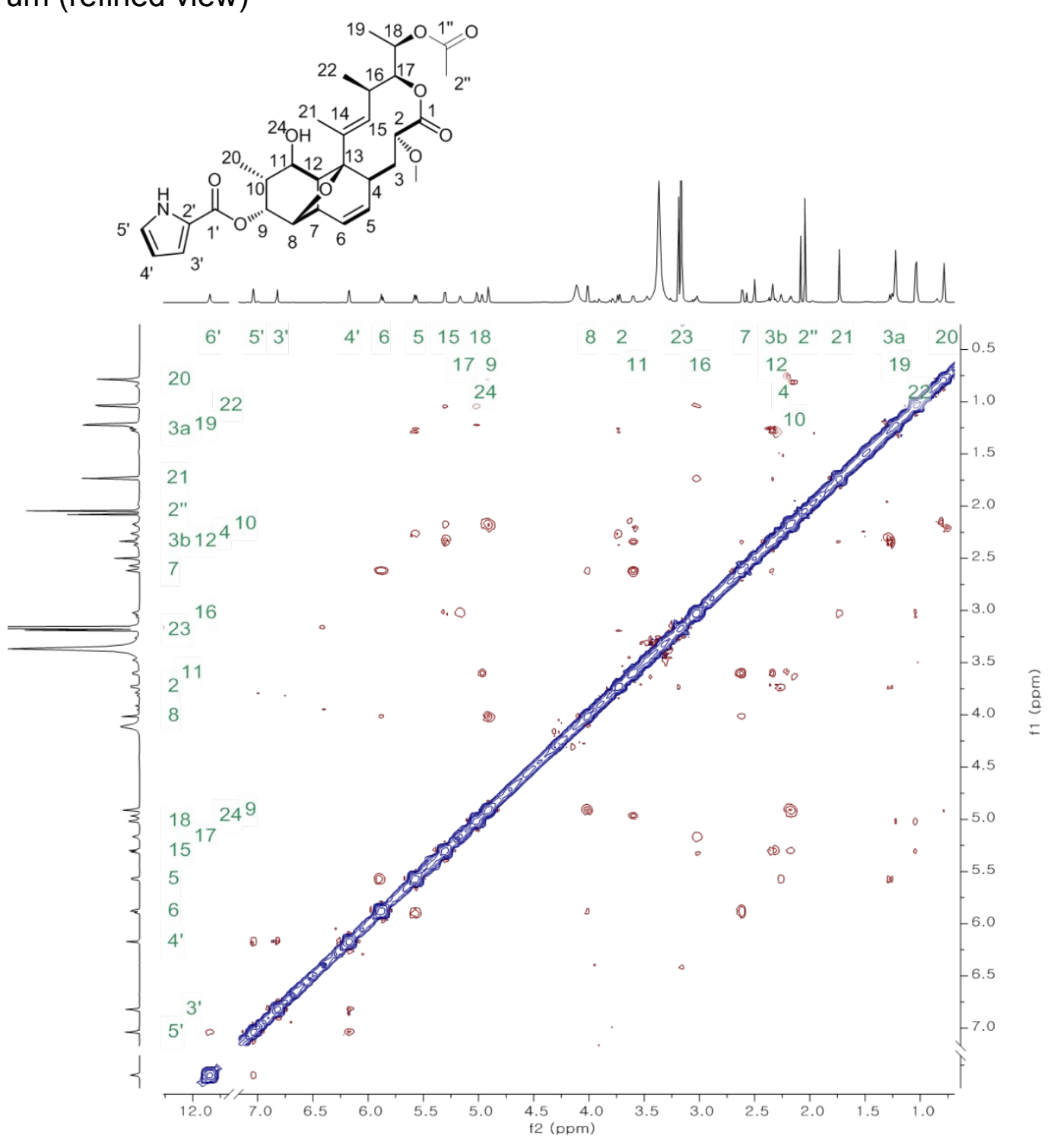


Figure S6. Different NMR spectrum of 18-O-acetyl nodusmicin (12)

A. ${ }^{1} \mathrm{H}$ NMR spectrum of 12 in $700 \mathrm{MHz}$, DMSO-d6

우ㅇㅜㅗ

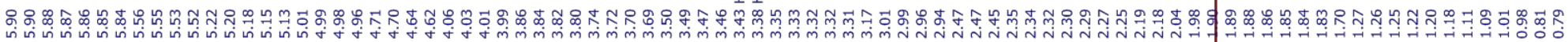

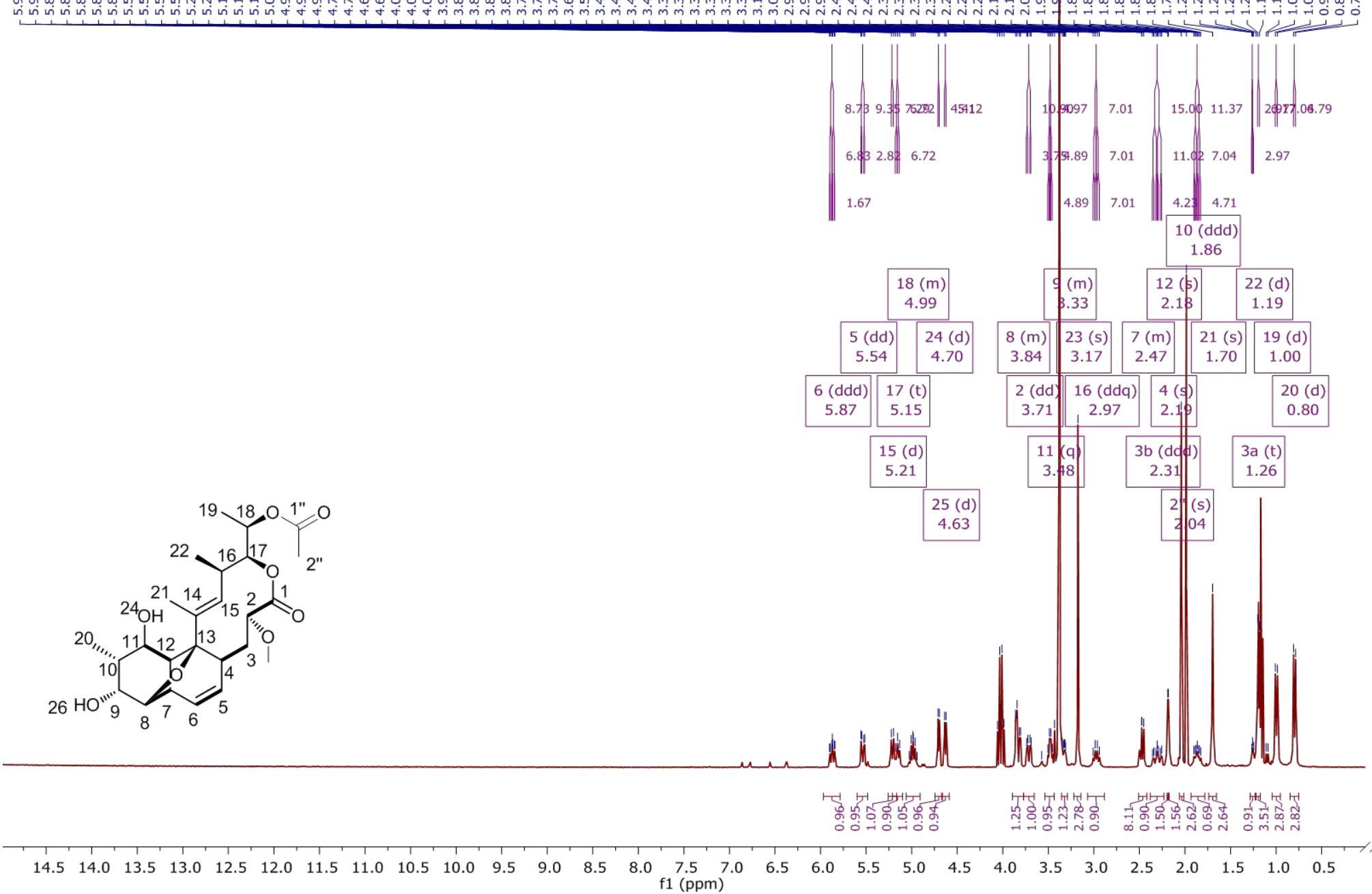


B. ${ }^{1} \mathrm{H}$ NMR spectrum of 12 in $700 \mathrm{MHz}$, DMSO-d6 (refined view, whereas EA denotes signals for ethyl acetate as impurity whose signals were excluded in structural analysis)

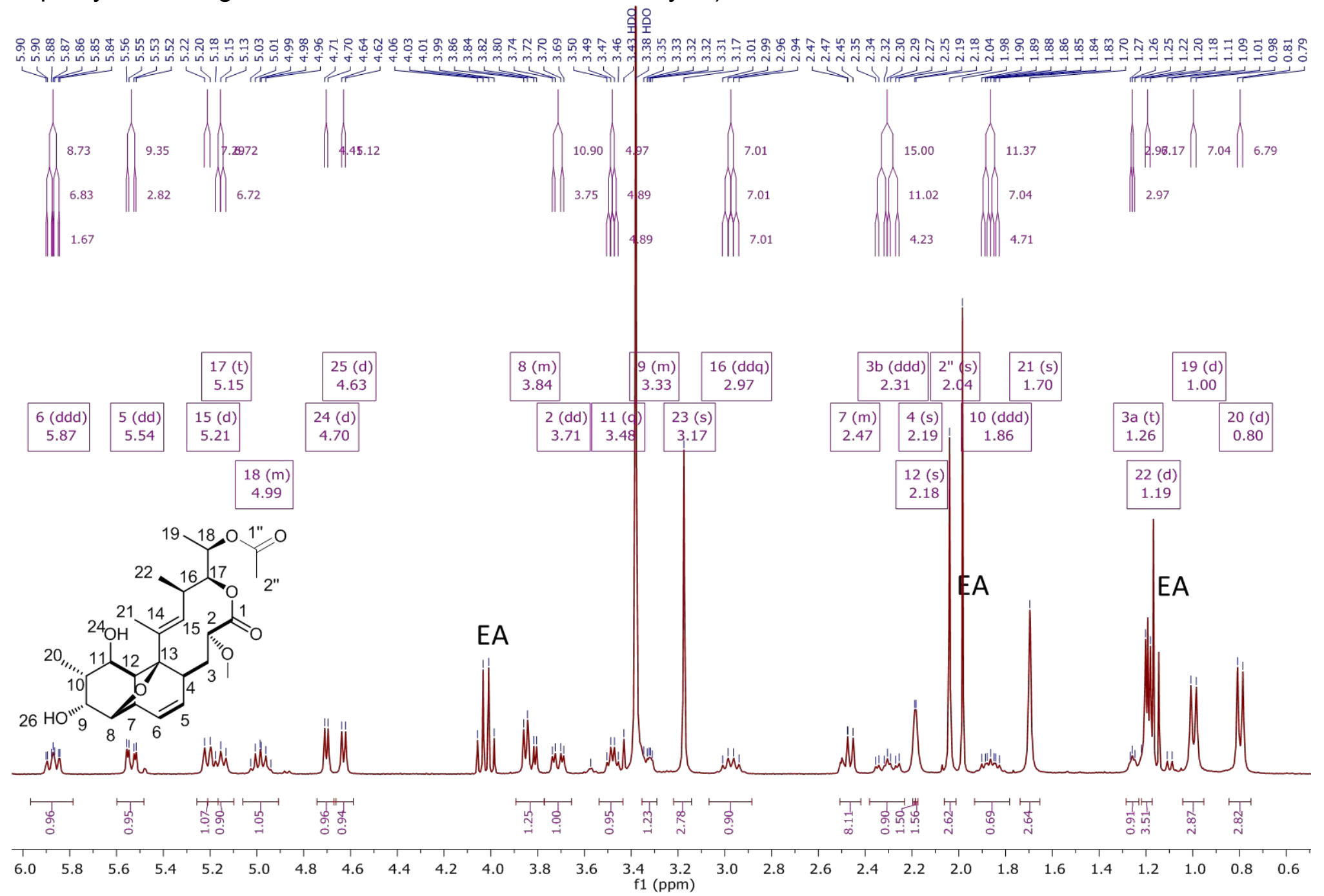


C. ${ }^{13} \mathrm{C}$ NMR spectrum of 12 in $176 \mathrm{MHz}$, DMSO-d6
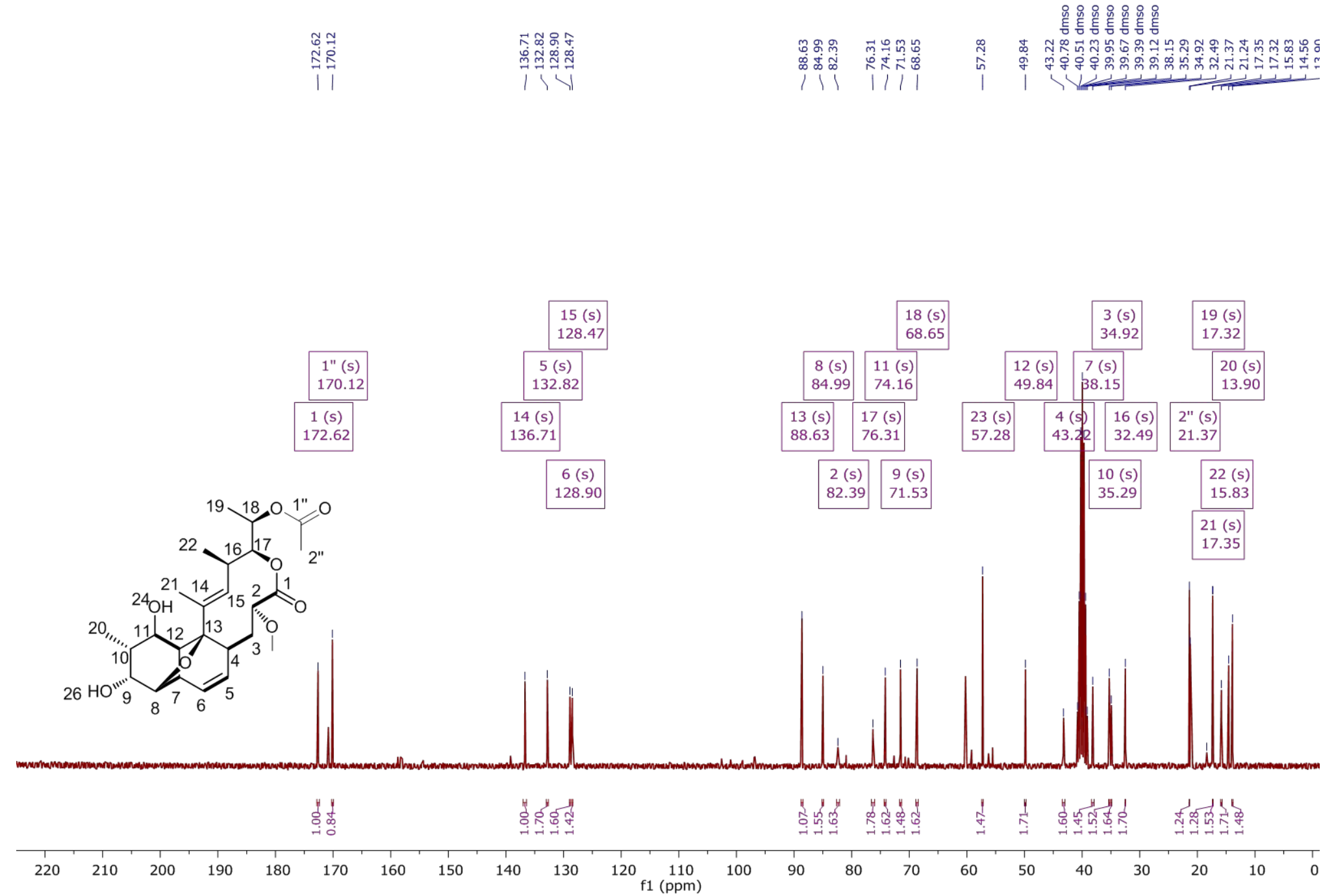
D. ${ }^{13} \mathrm{C}$ NMR spectrum of 12 in $176 \mathrm{MHz}$, DMSO-d6 (extended view whereas EA denotes signals for ethyl acetate as impurity whose signals were excluded in structural analysis)
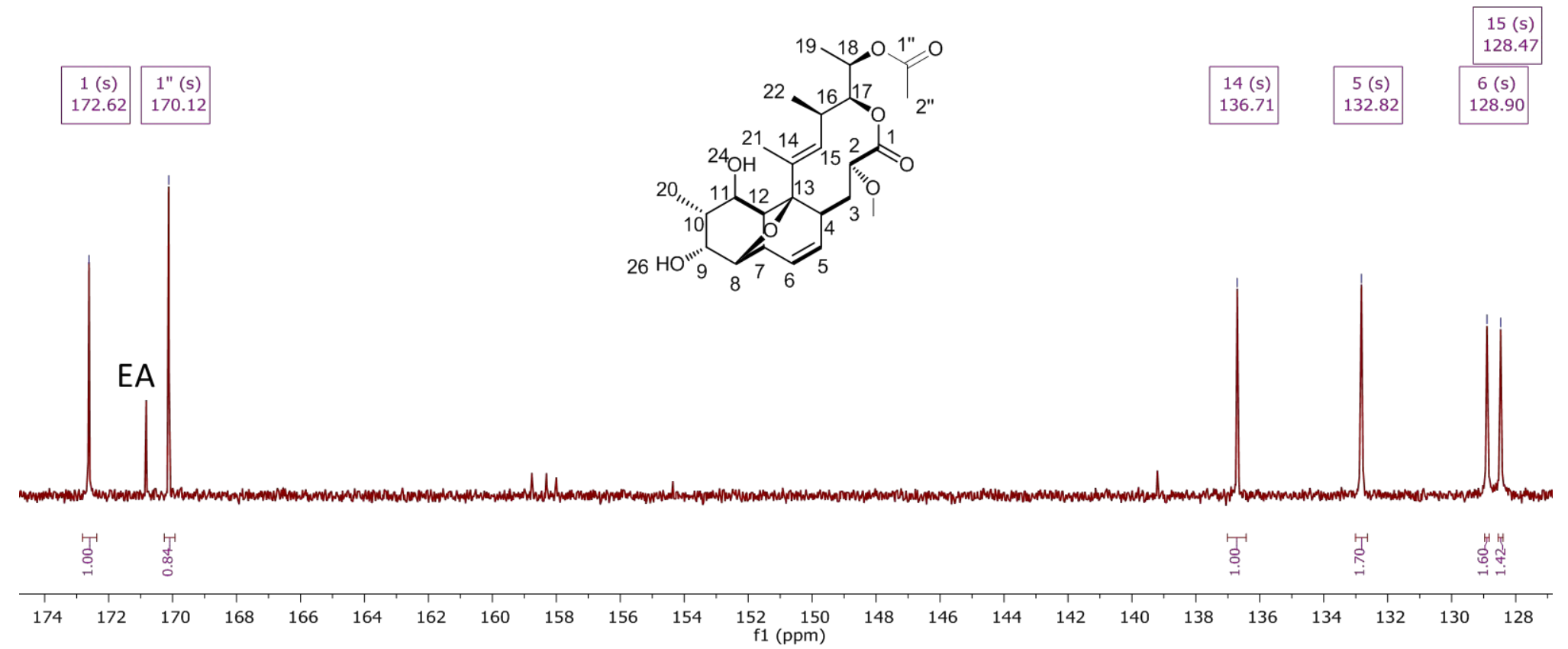
E. ${ }^{13} \mathrm{C}$ NMR spectrum of 12 in $176 \mathrm{MHz}$, DMSO-d6 (extended view whereas EA denotes signals for ethyl acetate as impurity whose signals were excluded in structural analysis)
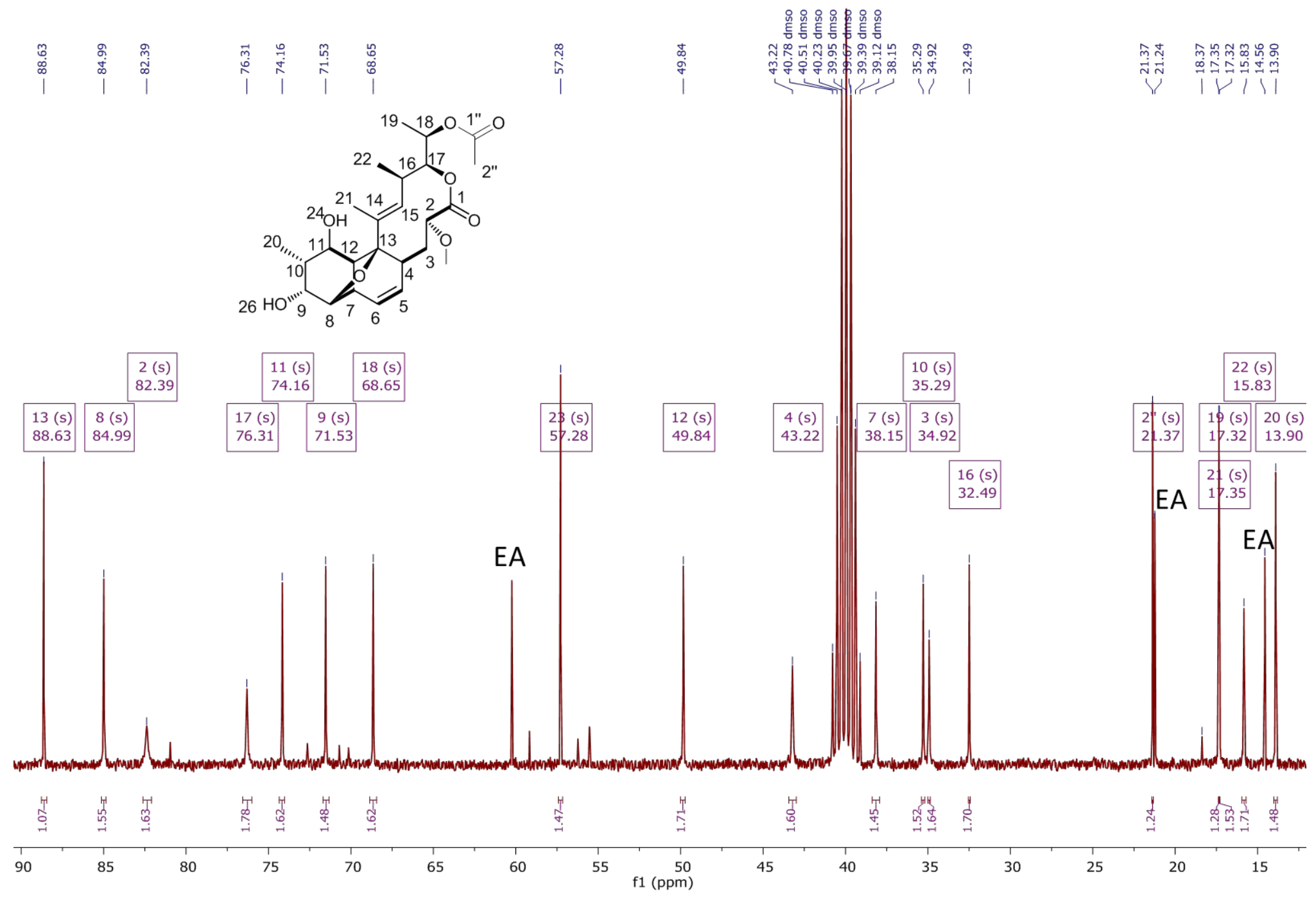
Figure S7. Analysis of heterologous expression of nargenicin A1. UPLC analysis of extract from different strains at 220nm (a)Wild type producer, Nocardia sp. CS682, (b) Heterologous production host, Streptomyces venezuelae YJ028/pCAP03nar ,(c) Control host for heterologous production, Streptomyces venezuelae YJ028/pCAP03, (d) EIC of MSMS analysis for nargenicin A1 from Streptomyces venezuelae YJ028/pCAP03-nar, (e)EIC of MS analysis for nargenicin A1 from Streptomyces venezuelae YJ028/pCAP03-nar (f) EIC of MS analysis for nargenicin A1 from Streptomyces venezuelae YJ028/pCAP03

(a)

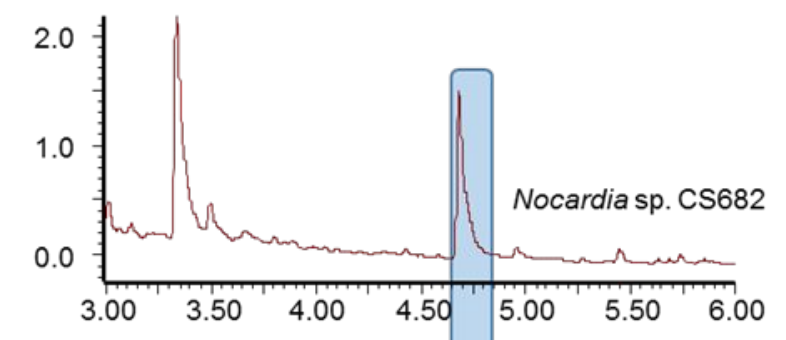

(b)

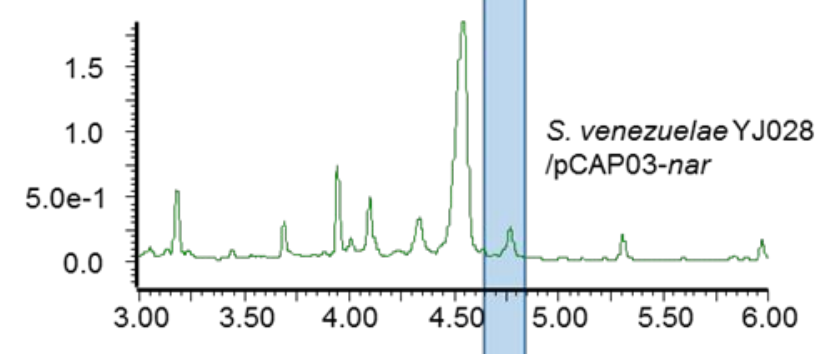

(c)

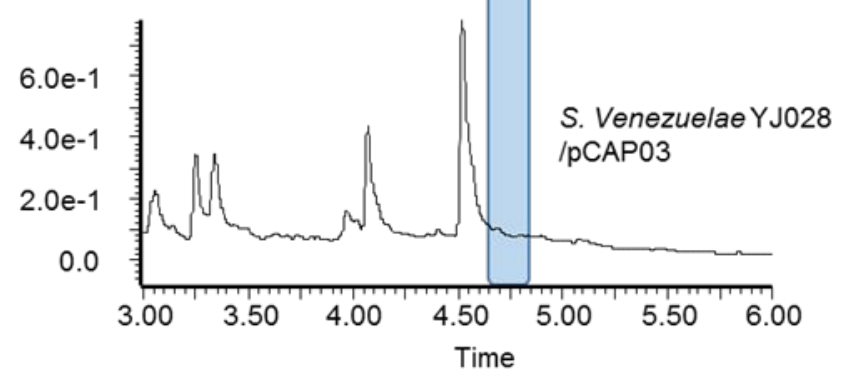

(d)

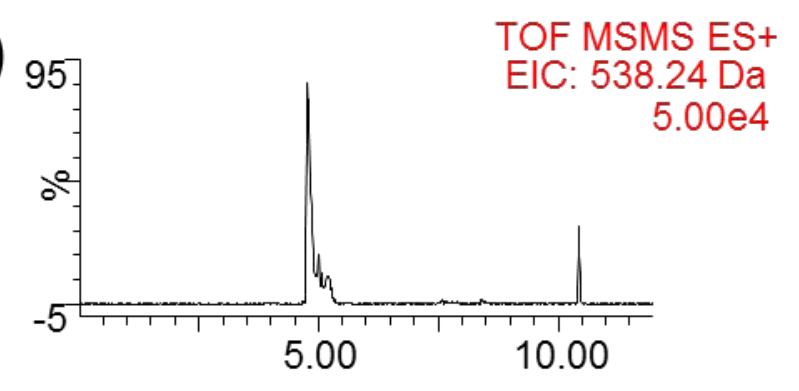

(e)

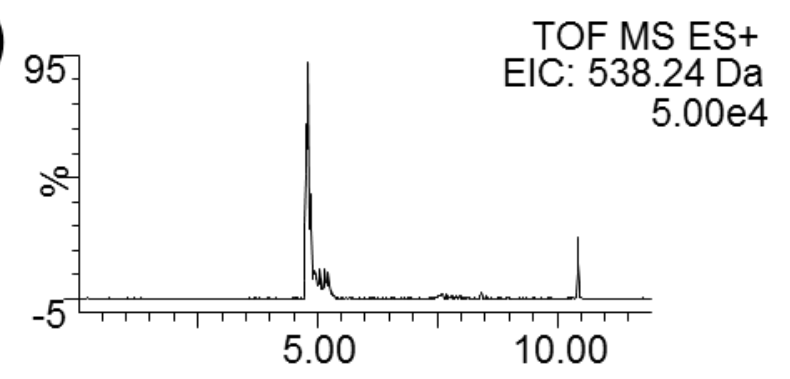

(f)

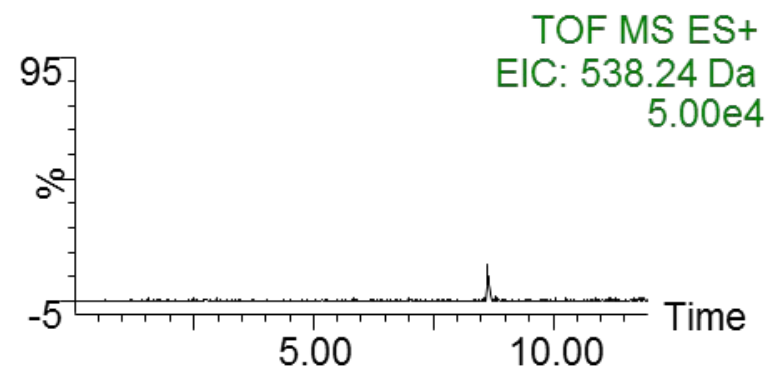


Figure S8. Mass analysis for nargenicin A1 from heterologous production host, Streptomyces venezuelae YJ028/pCAP03-nar (a) HR-QTOF- MSMS analysis for nargenicin A1 (b) QTOF-MS analysis for nargenicin A1

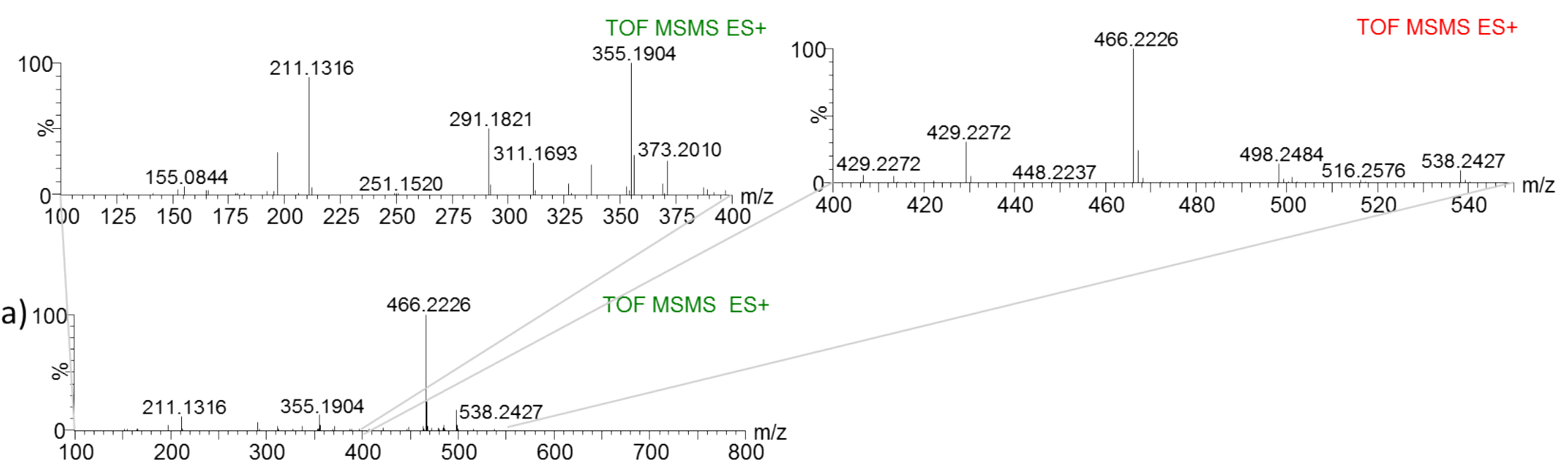

(b)

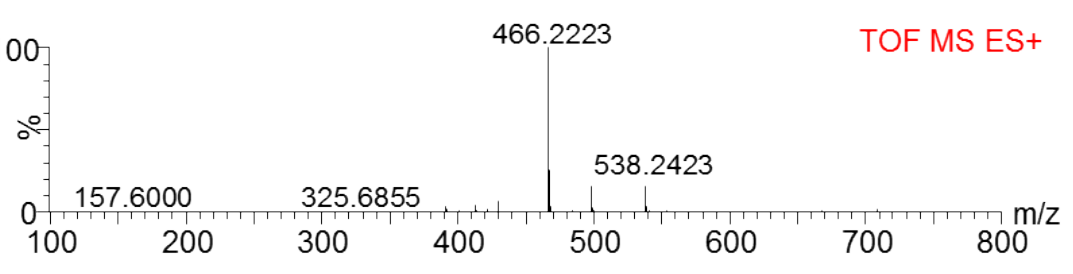


Figure S9. Confirmation of production of nargenicin A1 in heterologous host Streptomyces venezuelae YJ028/pCAP03nar based on mass fragmentation pattern observed in QTOF MSMS analysis

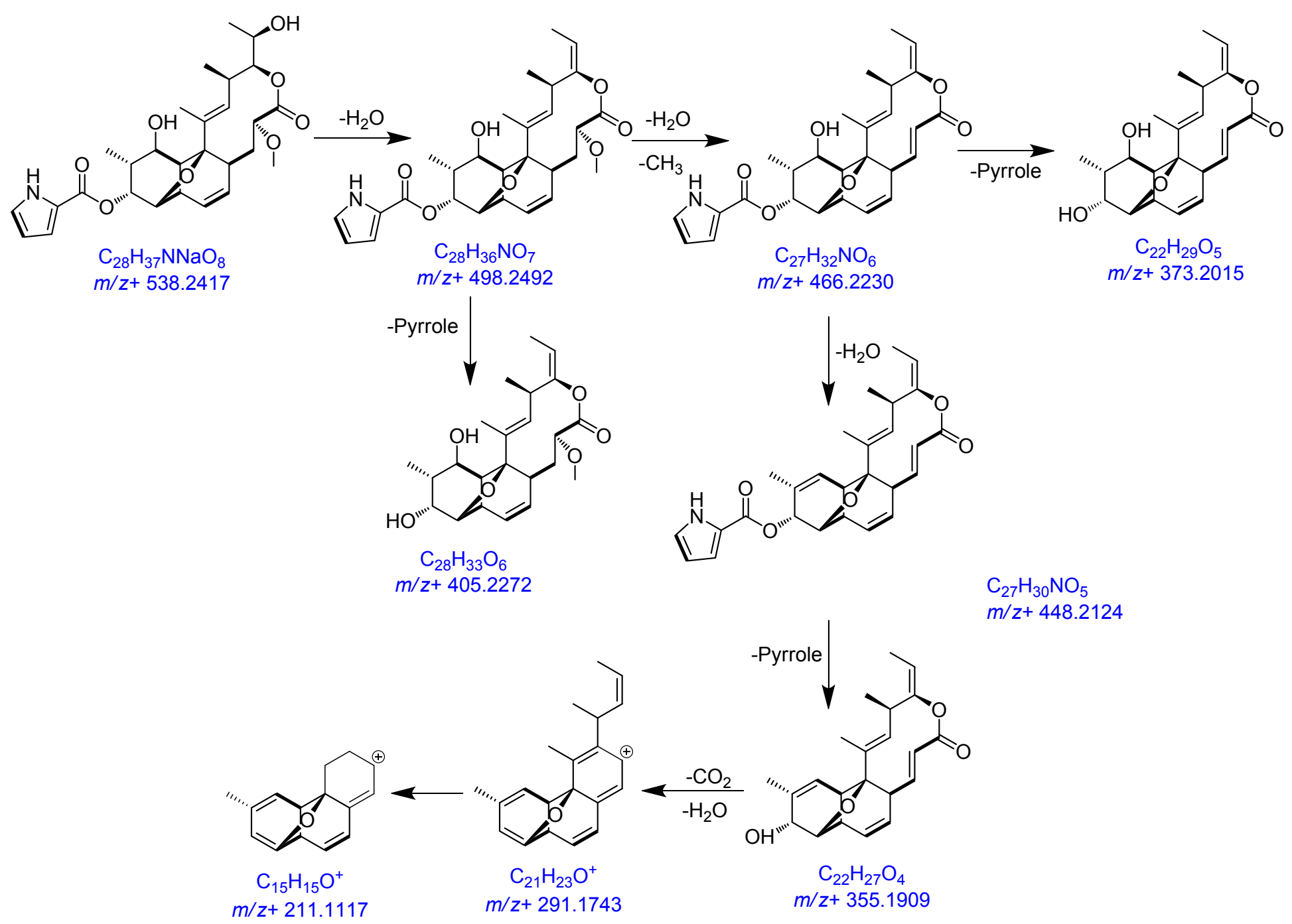


Figure S10. Construction of ngnP1 deletion mutant, Nocardia sp. $\Delta$ NgnP1 (a) Depiction of Nocardia sp. $\Delta$ NgnP1 (b) Gel electrophoresis of PCR products. DNA templates were from wild type Nocardia sp. CS682 (W1), deletion mutant Nocardia sp. $\triangle \mathrm{NgnP} 1$ (D1) and marker (M1).

(a)

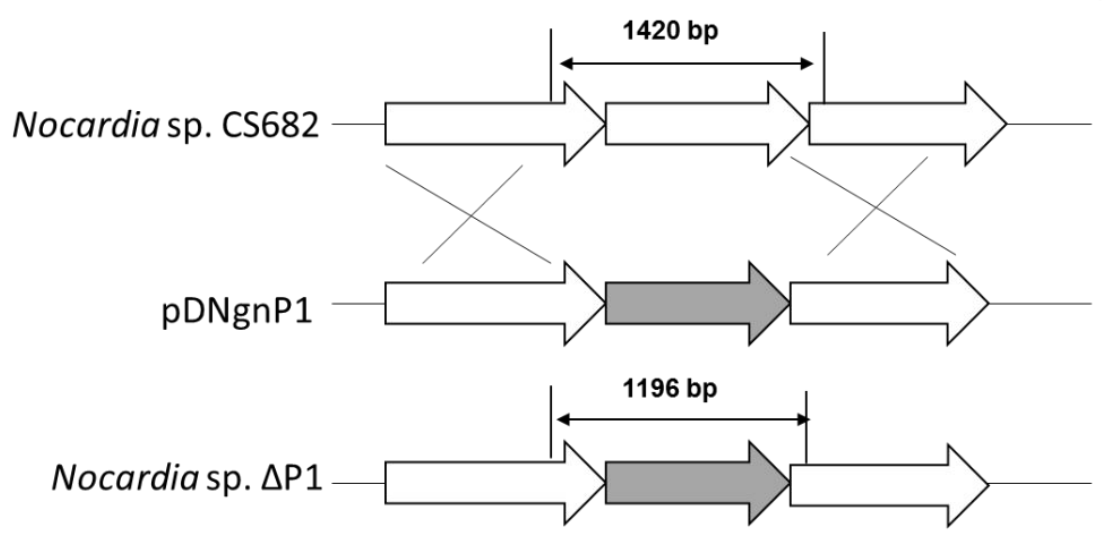

(b)

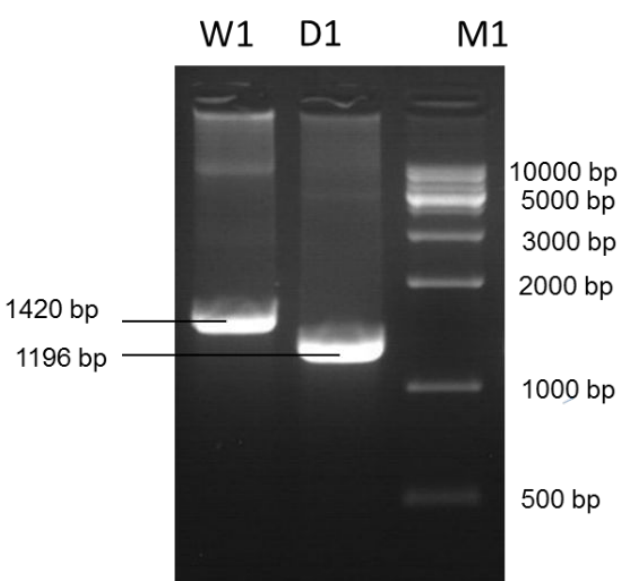


Figure S11. HPLC chromatogram of extract from different strains.(a) Wild type, Nocardia sp. CS682, (b) deletion mutant Nocardia sp. $\Delta \mathrm{NgnP} 1$ and (c)complementation strain Nocardia sp. $\Delta \mathrm{NgnP} 1 / \mathrm{p} 18 \mathrm{NgnP} 1$
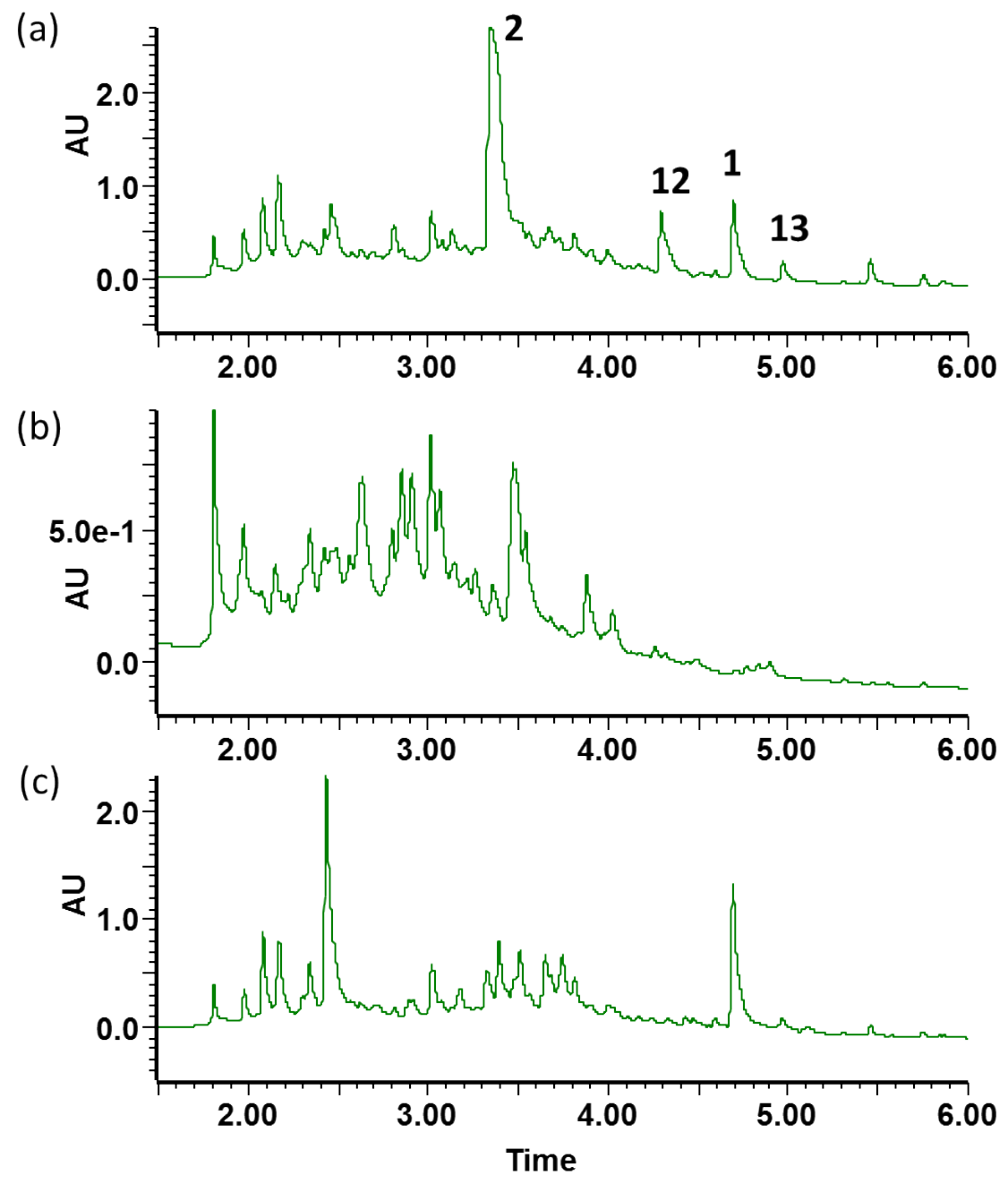
Figure S12. HR-QTOF Mass chromatogram of compound 6 from Nocardia sp. $\triangle \mathrm{NgnP} 1$ (a) QTOF MSMS analysis (b) QTOF MS analysis (c) EIC chromatogram for MSMS analysis of compound 6 in Nocardia sp. $\Delta$ NgnP1, (d) EIC chromatogram for MS analysis of compound 6 in Nocardia sp. $\triangle \mathrm{NgnP} 1$, (e) EIC chromatogram for MS analysis of compound 6 in wild type, Nocardia sp. CS682 and (f) Confirmation of 6 based on mass analysis

(a)

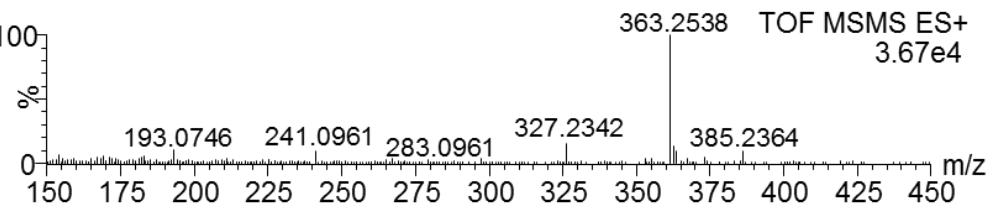

(b)

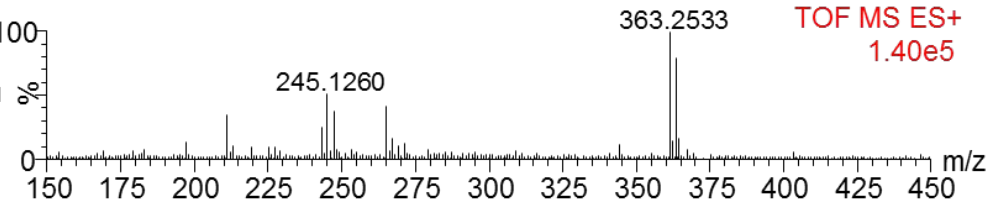

(c)

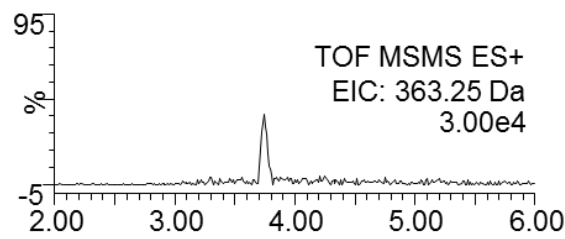

(d)

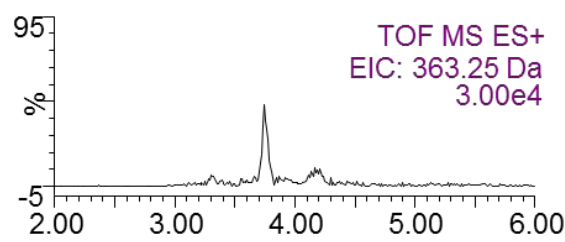

(e)

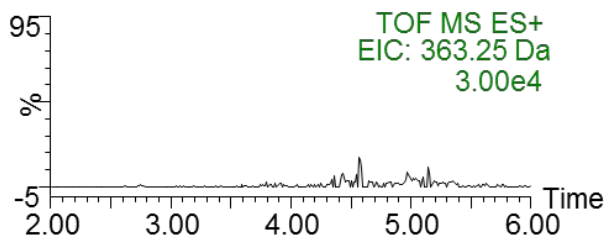

(f)

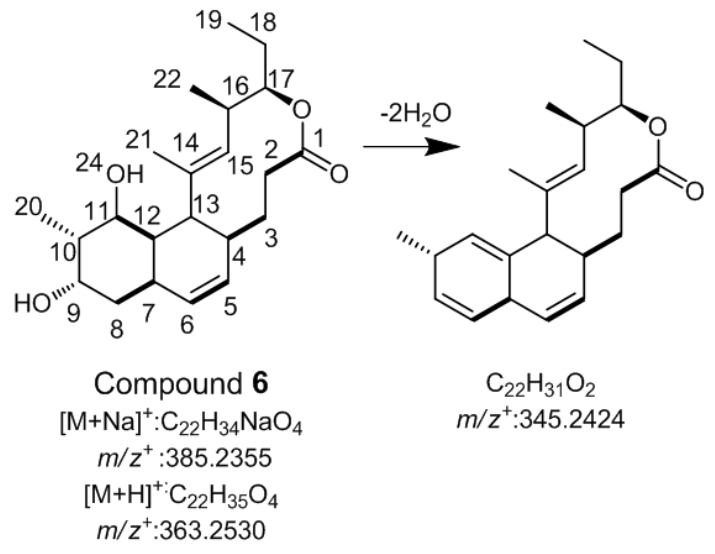


Figure S13. HR-QTOF Mass chromatogram of compound 7 from Nocardia sp. $\triangle \mathrm{NgnP} 1$ (a) QTOF MSMS analysis (b) QTOF MS analysis (c) EIC chromatogram for MSMS analysis of compound 7 in Nocardia sp. $\Delta$ NgnP1, (d) EIC chromatogram for MS analysis of compound 7 in Nocardia sp. $\triangle \mathrm{NgnP} 1$, (e) EIC chromatogram for MS analysis of compound 7 in wild type, Nocardia sp. CS682 and (f) Confirmation of 7 based on mass analysis

(a)

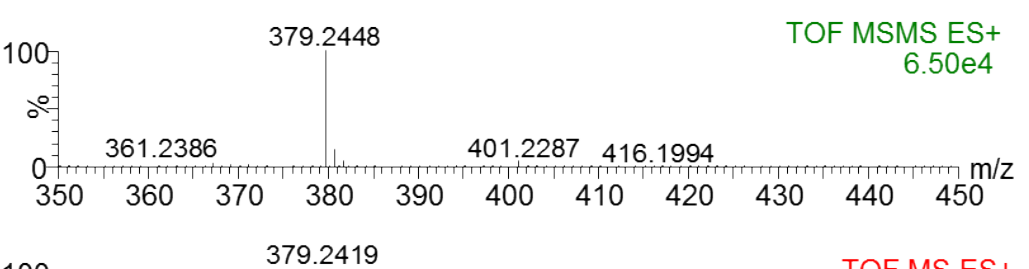

(b)

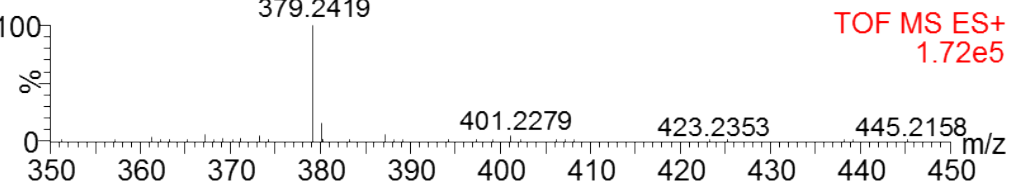

(c)

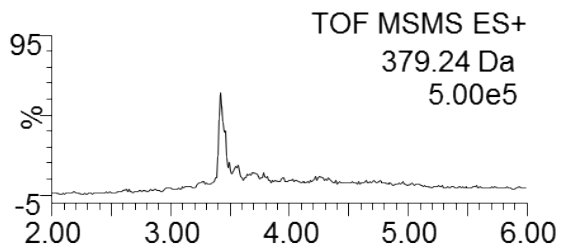

(d)

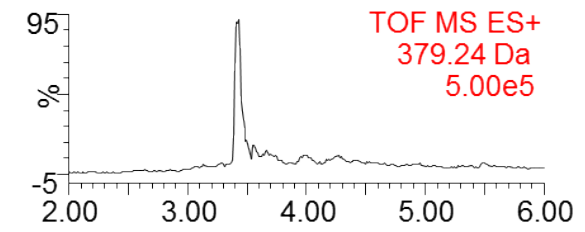

(e)

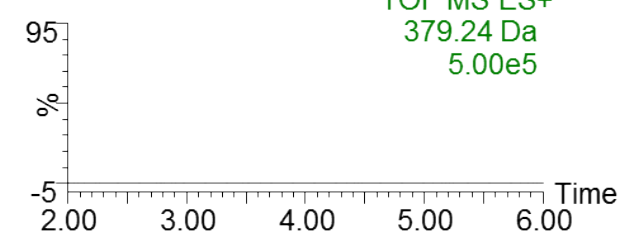

(f)

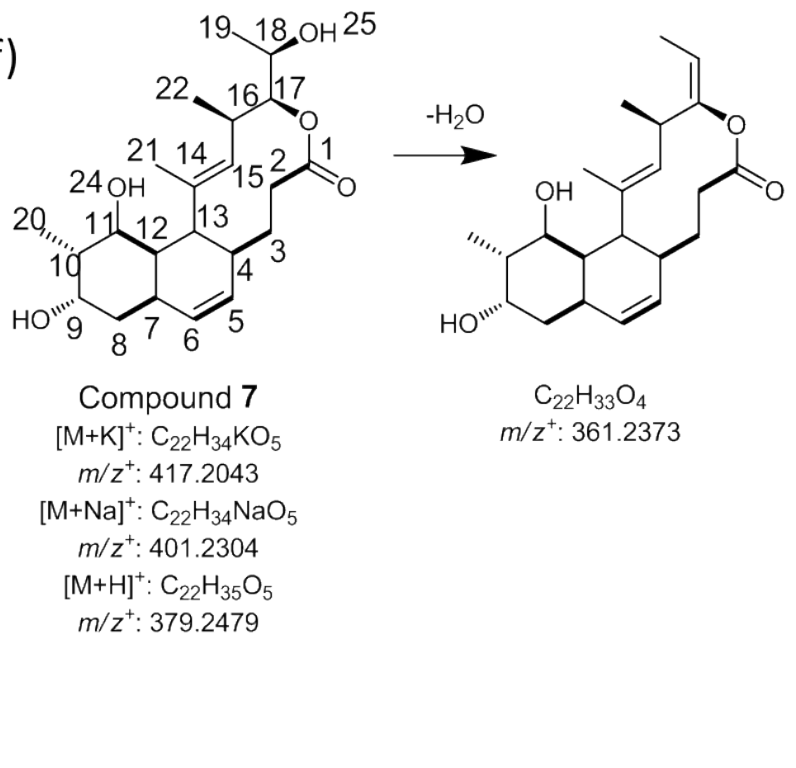


Figure S14. Construction of $n g n M$ deletion mutant, Nocardia sp. $\Delta$ NgnM (a) Depiction of $n g n M$, (b) Gel electrophoresis of PCR products. DNA templates were from deletion mutant Nocardia sp. $\Delta$ NgnM(D2), wild type Nocardia sp. CS682 (W2), and marker (M2).

(a)

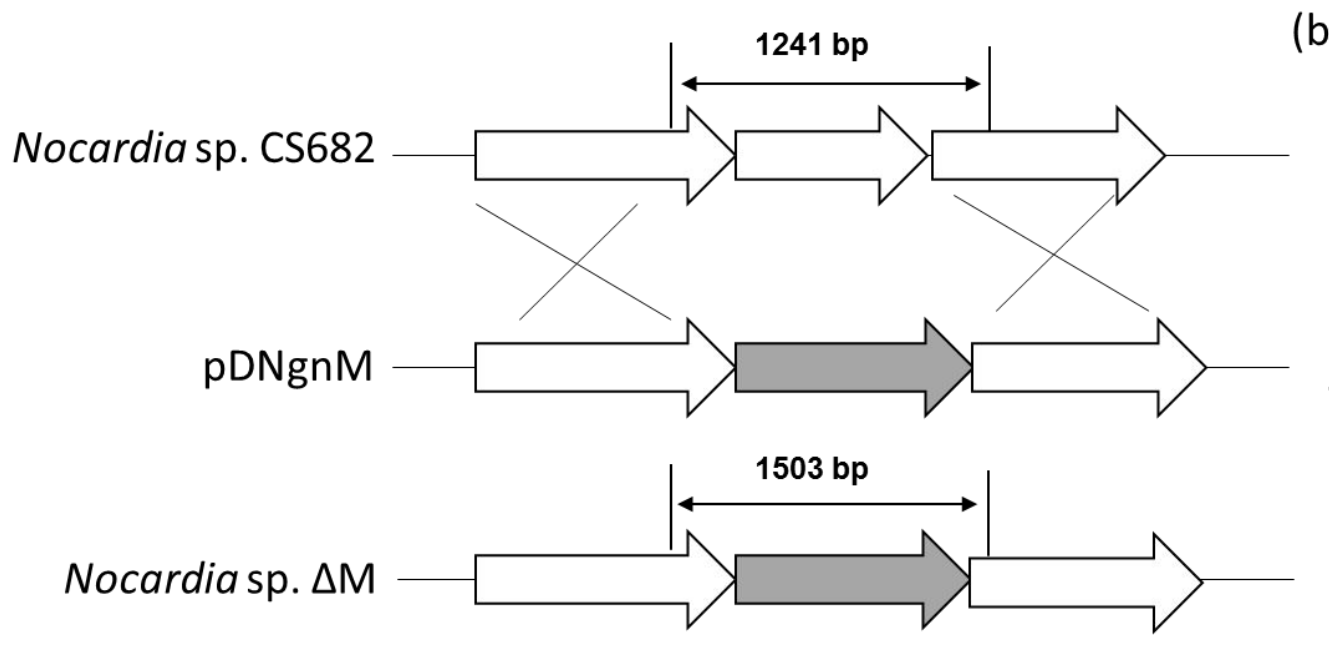

(b)

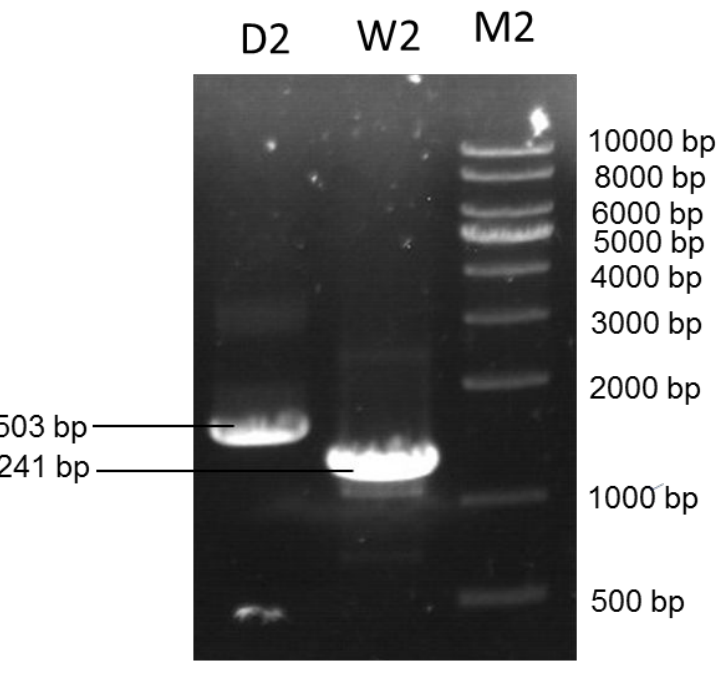


Figure S15. HPLC chromatogram of extract from different strains (a) Wild type, Nocardia sp. CS682, (b) deletion mutant Nocardia sp. $\Delta \mathrm{NgnM}$ and (c) complementation strain Nocardia sp. $\Delta \mathrm{NgnM} / \mathrm{p} 18 \mathrm{NgnM}$
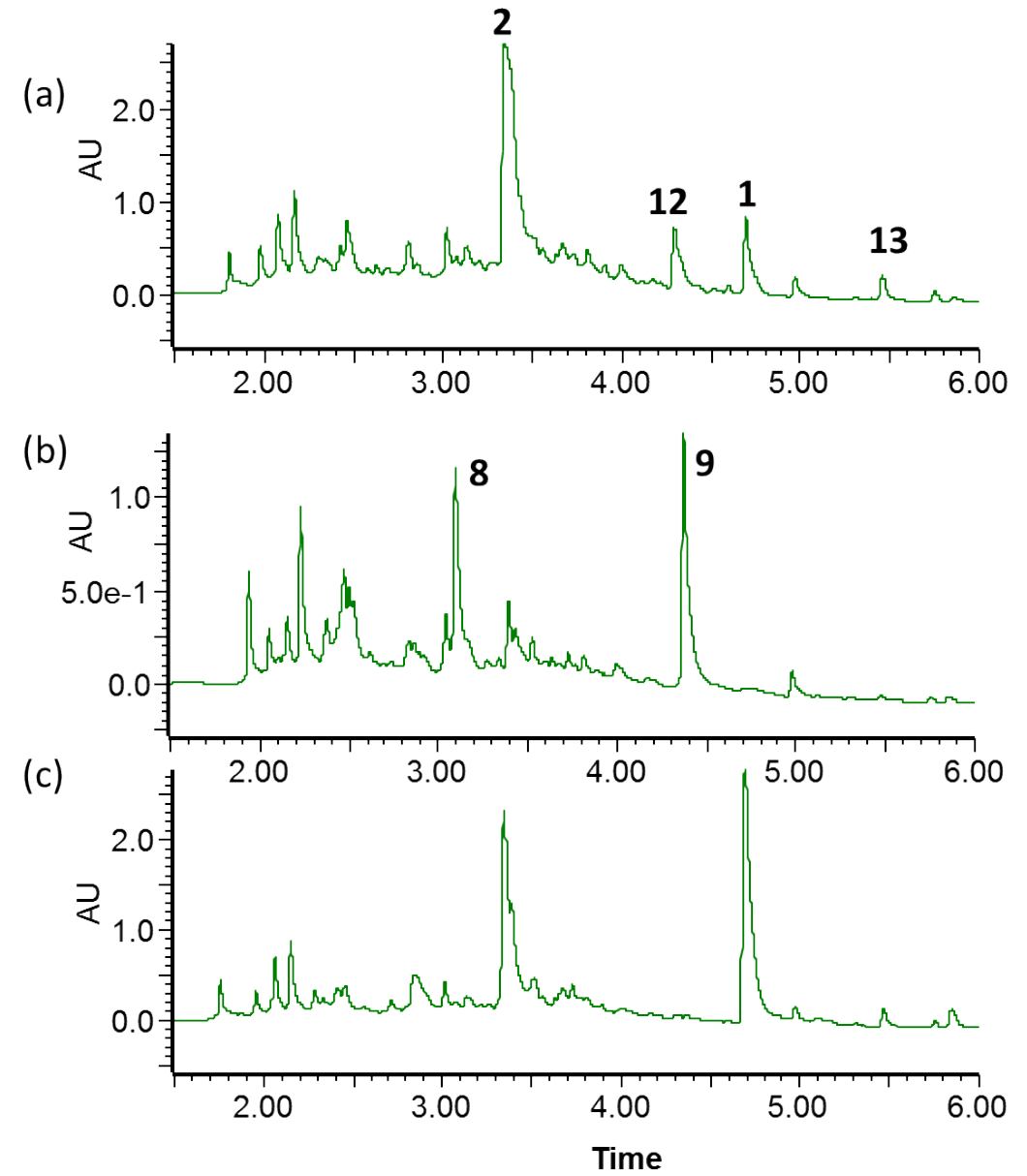
Figure S16. HR-QTOF Mass chromatogram of compounds from Nocardia sp. $\Delta \mathrm{NgnM}$ (a) Mass chromatogram for 23 demethyl 8, 13 deoxynodusmicin (8) and (b) 23 demethyl 8, 13 deoxynargenicin (9). UV absorbance of different compounds (c) 8 and (d) 9

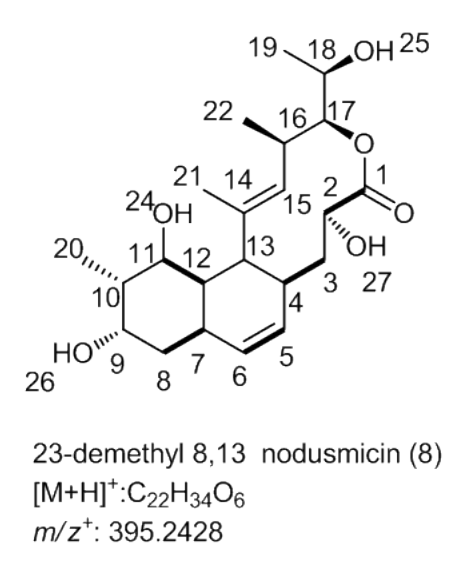

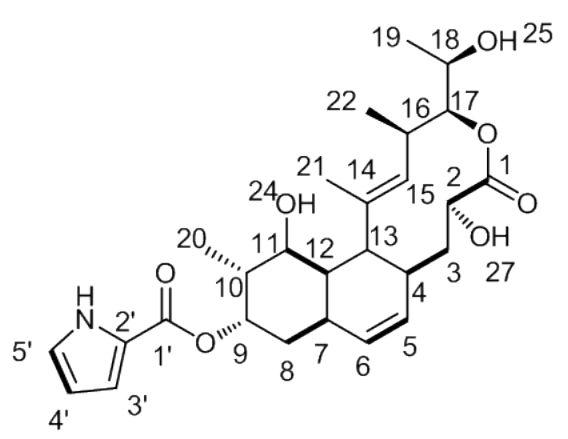

23-demethyl 8,13 nargenicin (9) $[\mathrm{M}+\mathrm{H}]^{+}: \mathrm{C}_{27} \mathrm{H}_{38} \mathrm{NO}_{7}$ $\mathrm{m} / \mathrm{z}^{+}: 488.2643$
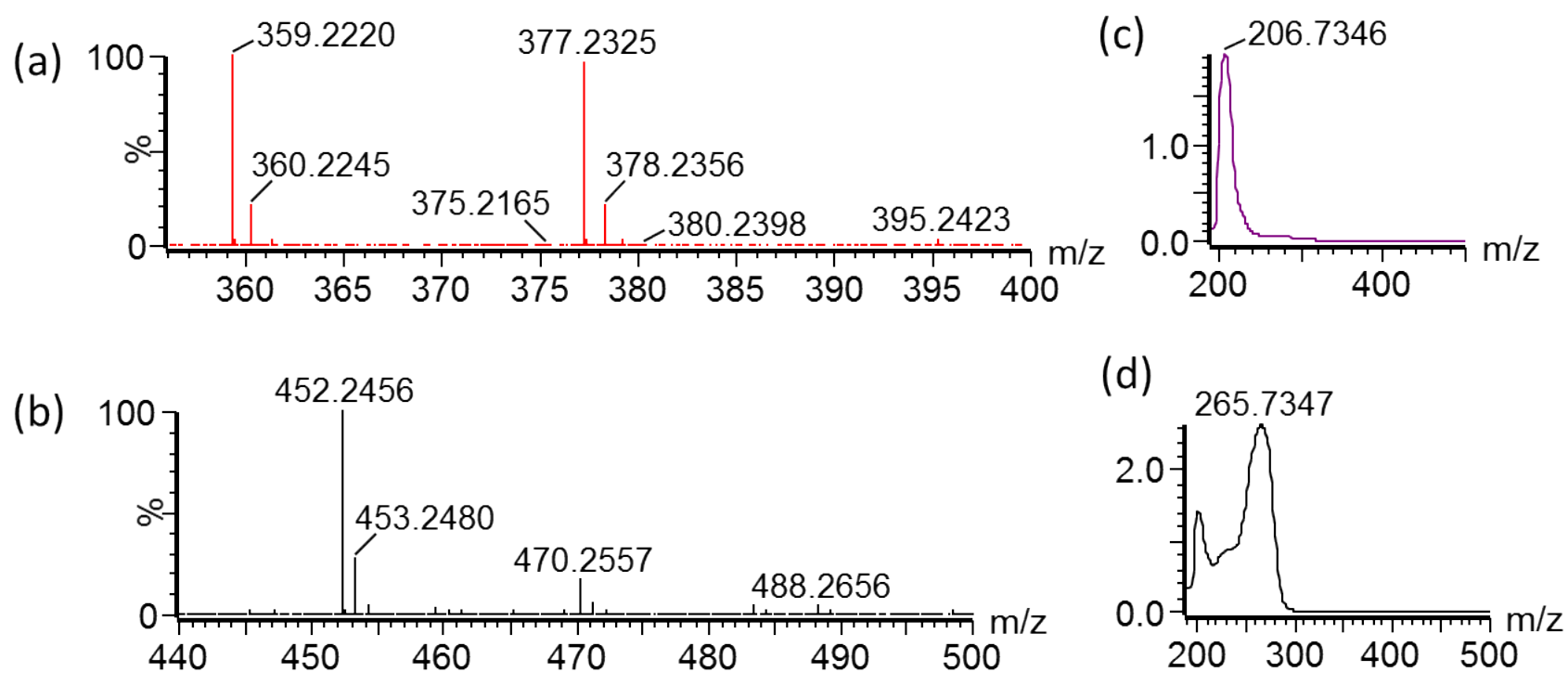
Figure S17. Different NMR spectrum of 23-demethyl-8, 13 deoxynodusmicin (8)

\section{A. ${ }^{1} \mathrm{H}$ NMR spectrum of 8 in $700 \mathrm{MHz}$, DMSO-d6}

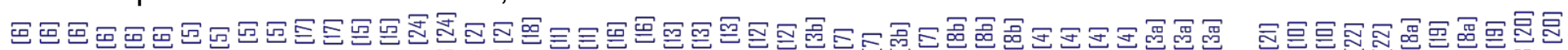

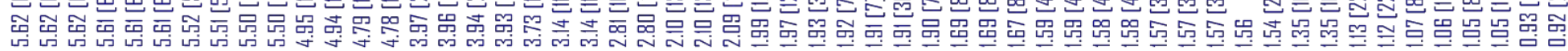

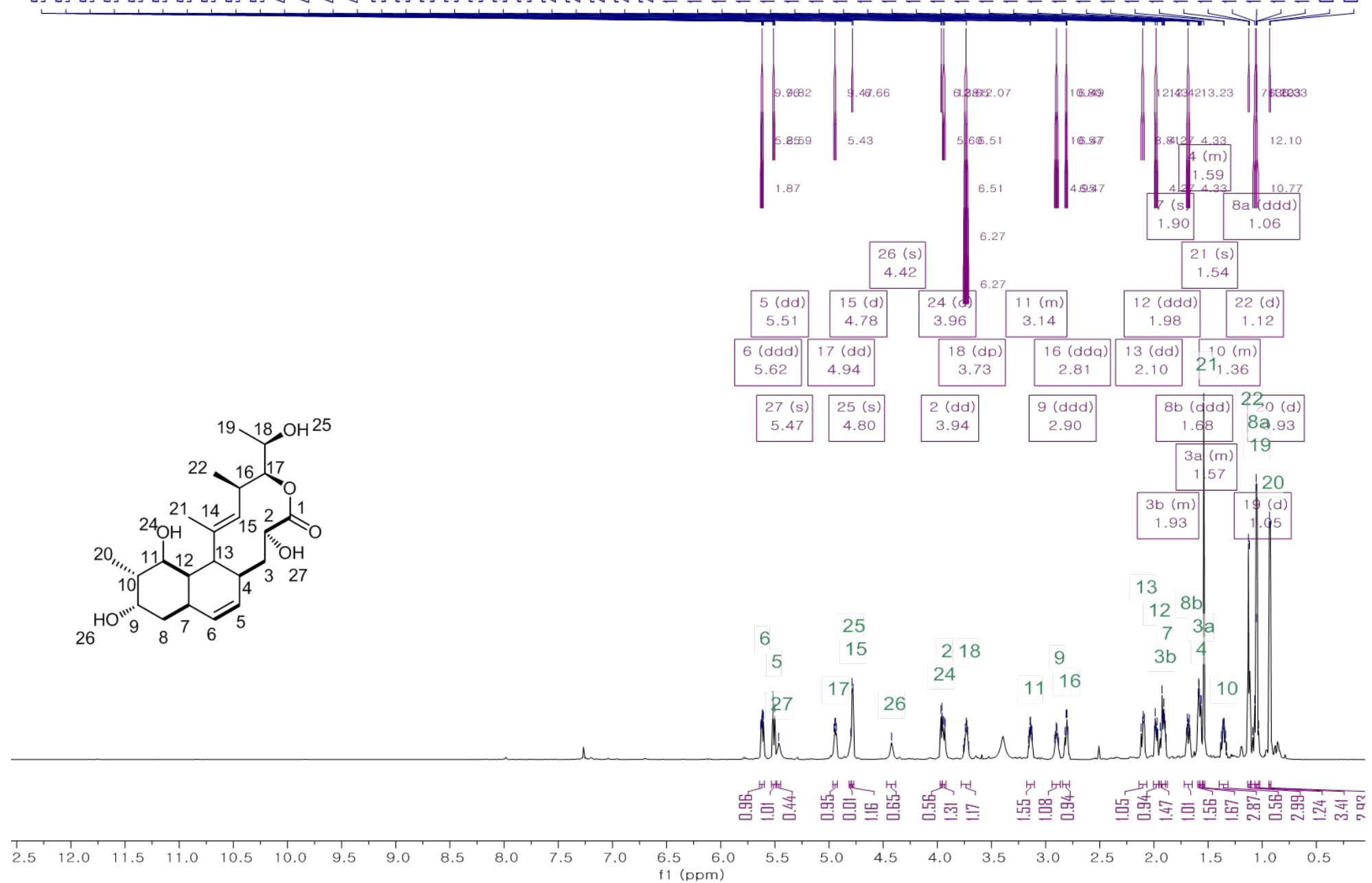


B. ${ }^{1} \mathrm{H}$ NMR spectrum of 8 in $700 \mathrm{MHz}$, DMSO-d6 (refined view)

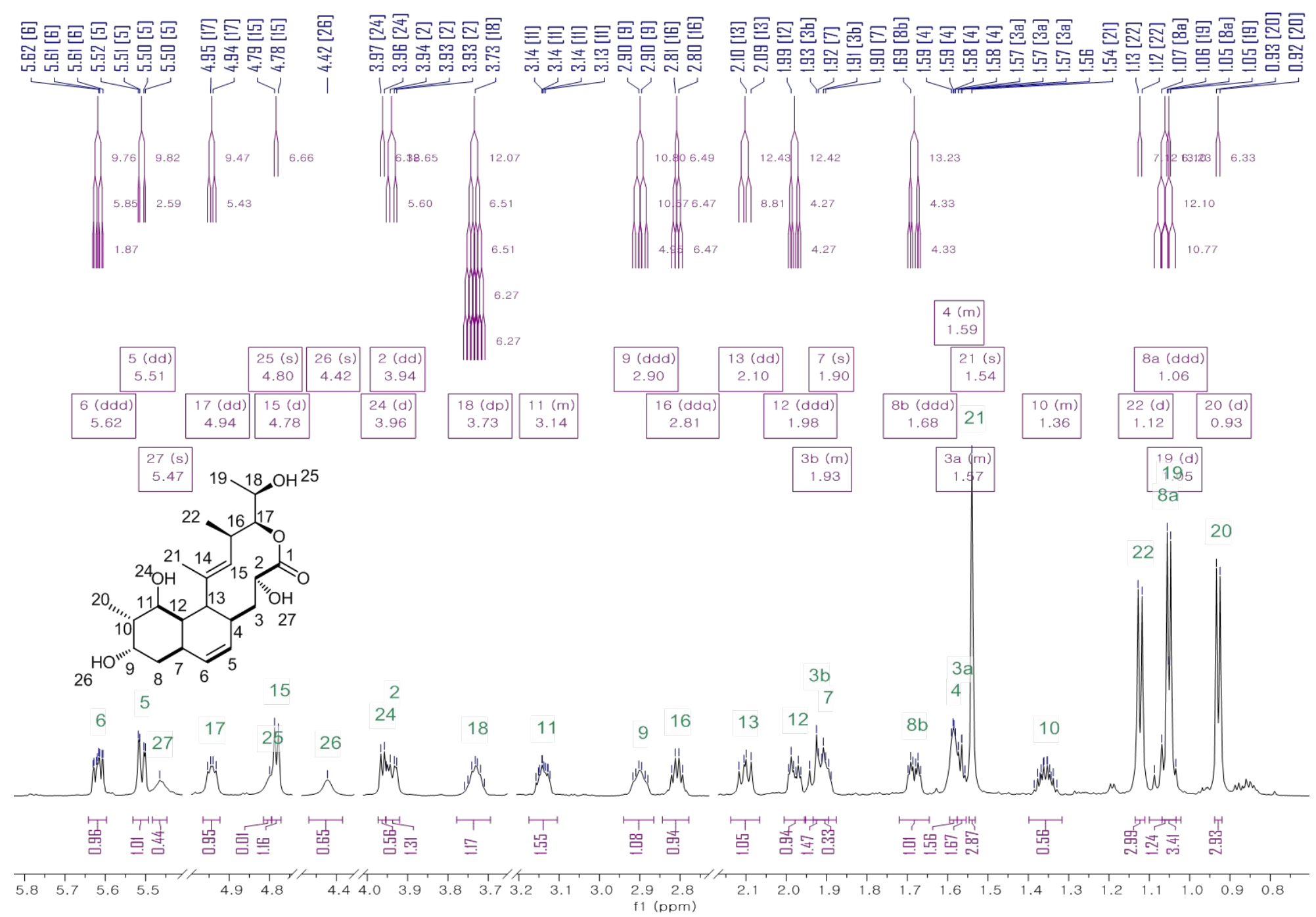


C. ${ }^{13} \mathrm{C}$ NMR spectrum of 8 in $176 \mathrm{MHz}$, DMSO-d6

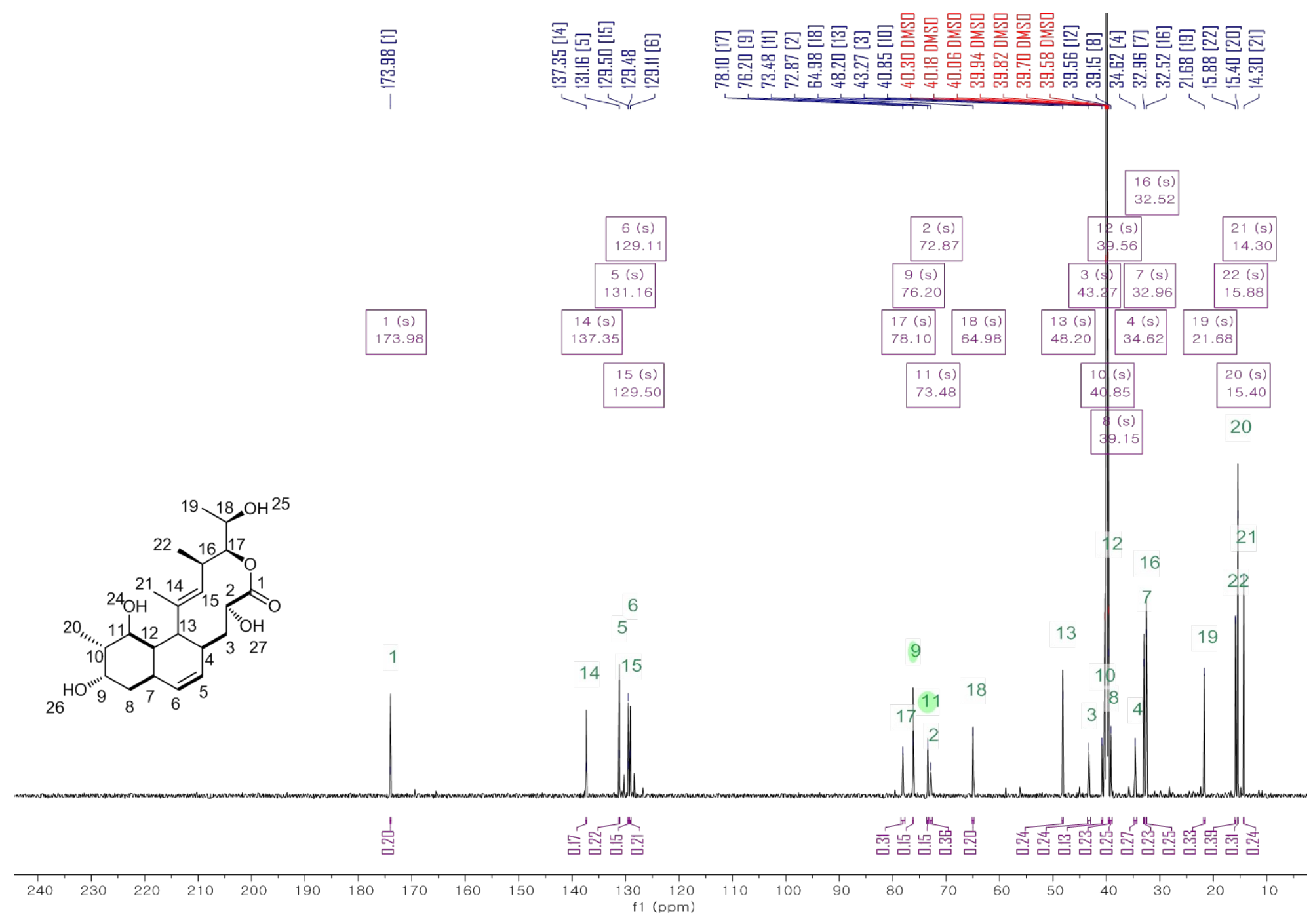


D. ${ }^{13} \mathrm{C}$ NMR spectrum of 8 in $176 \mathrm{MHz}$, DMSO-d6 (refined view)

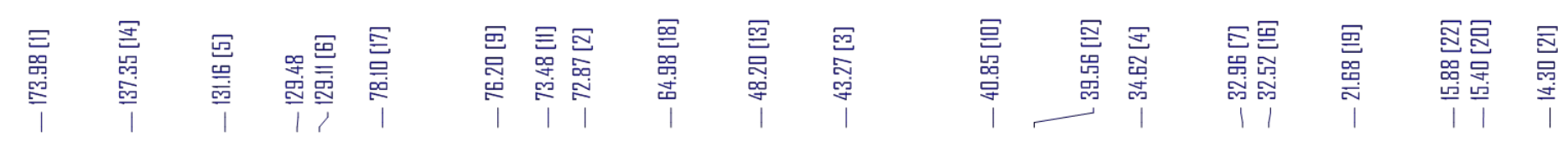
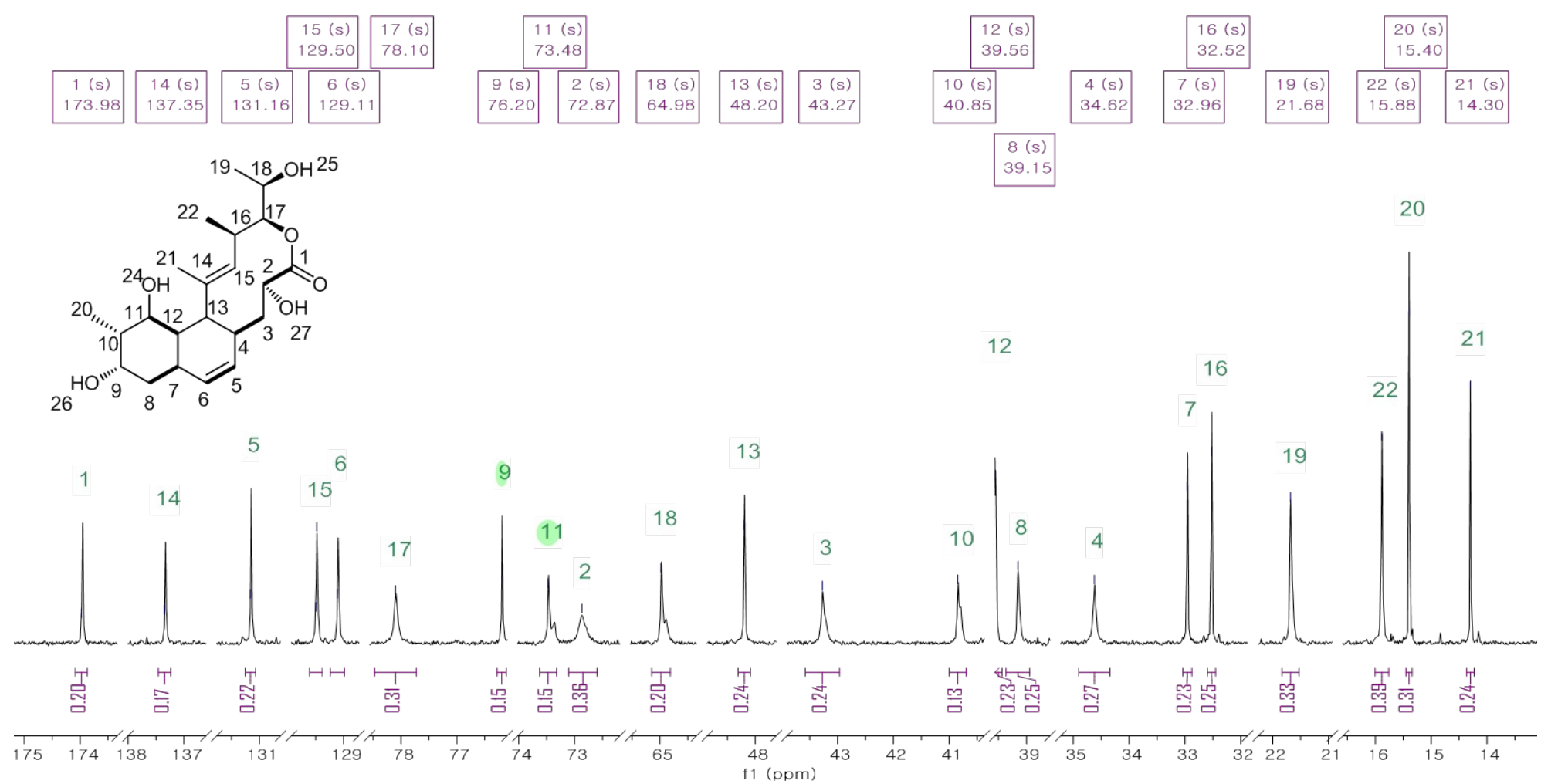
E. COSY NMR spectrum

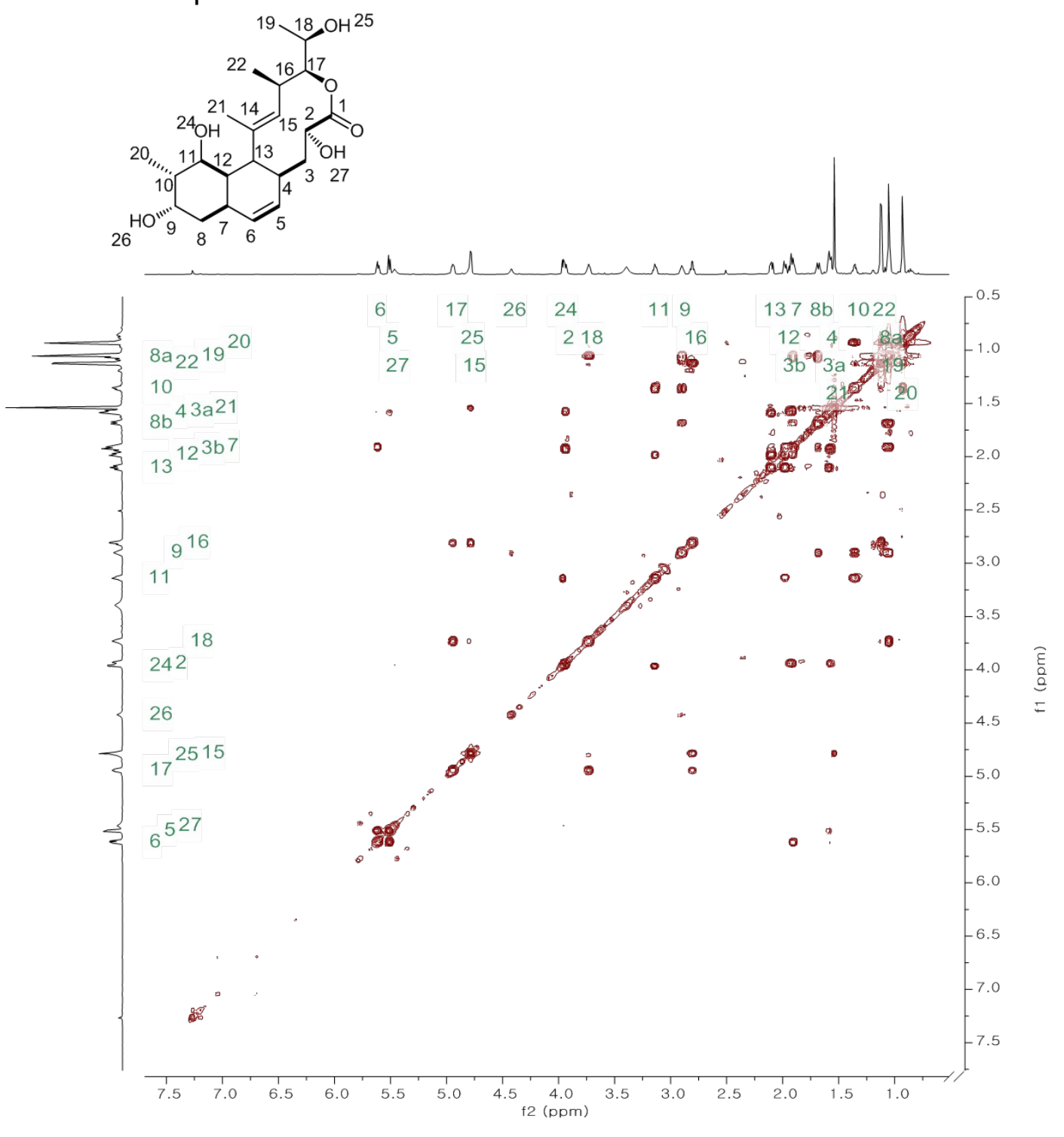


F. COSY NMR spectrum (refined view)

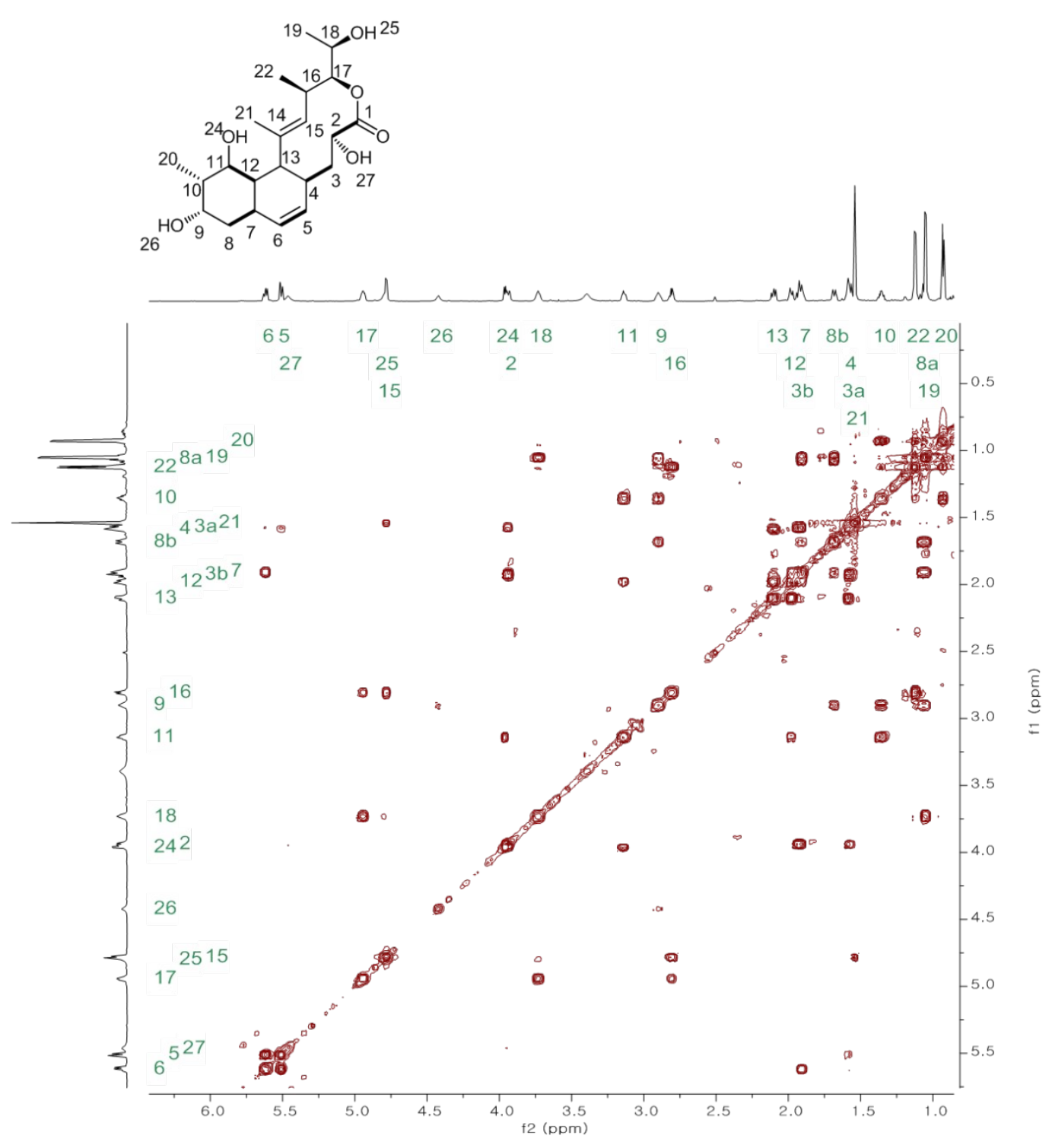


G. HSQC-DEPT NMR spectrum

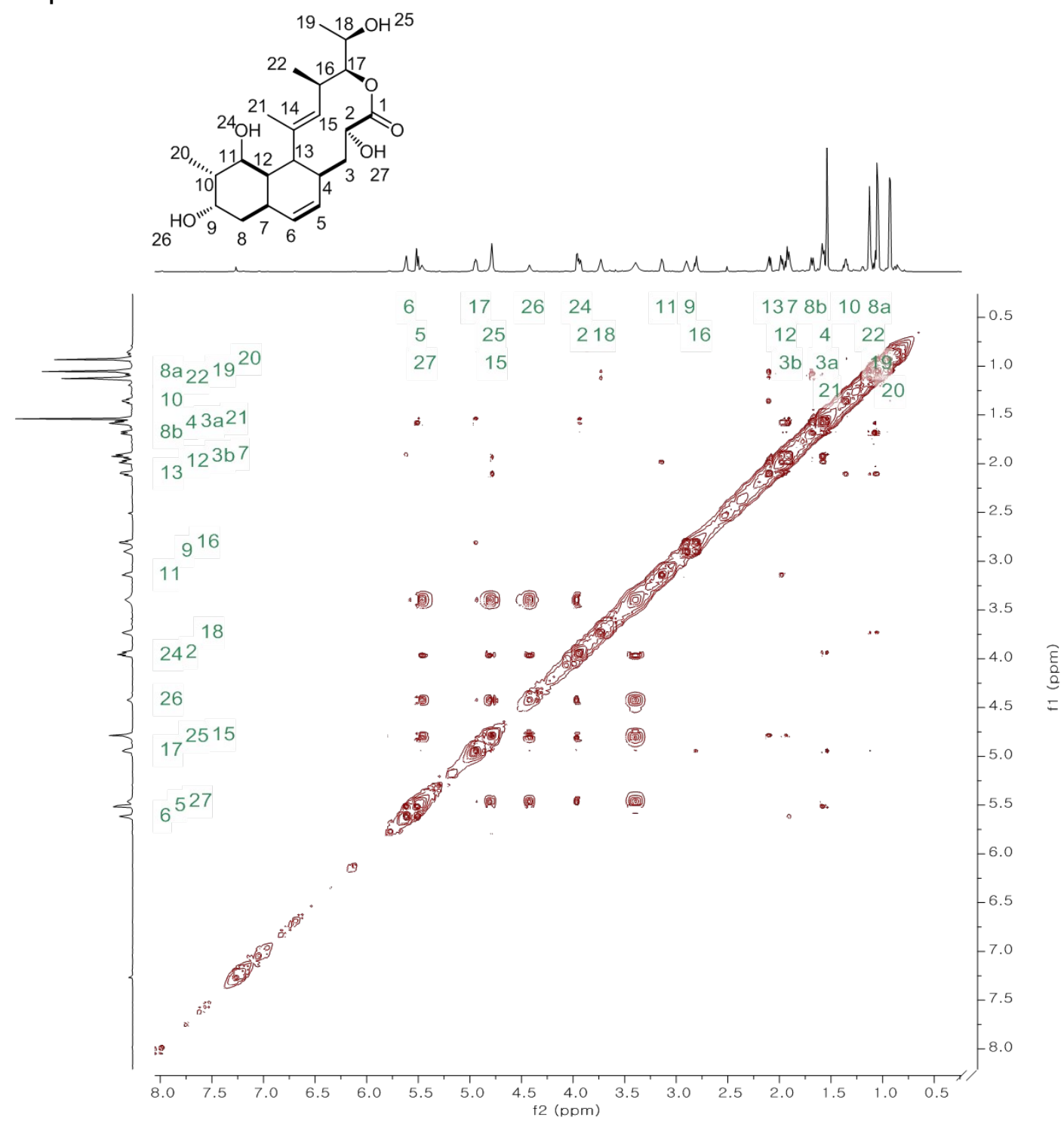


H. HSQC-DEPT NMR spectrum (refined view)

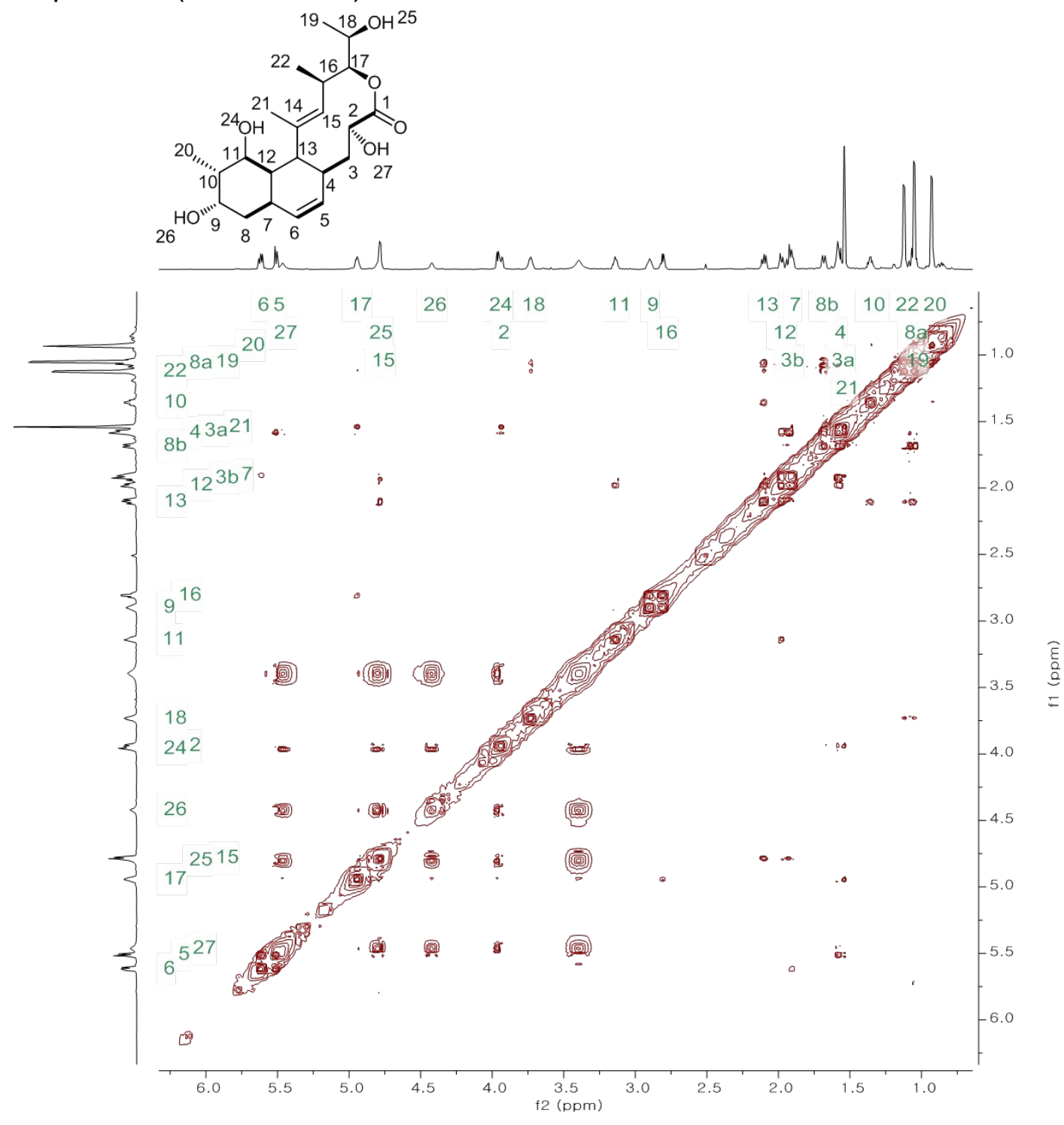




\section{HMBC NMR spectrum}

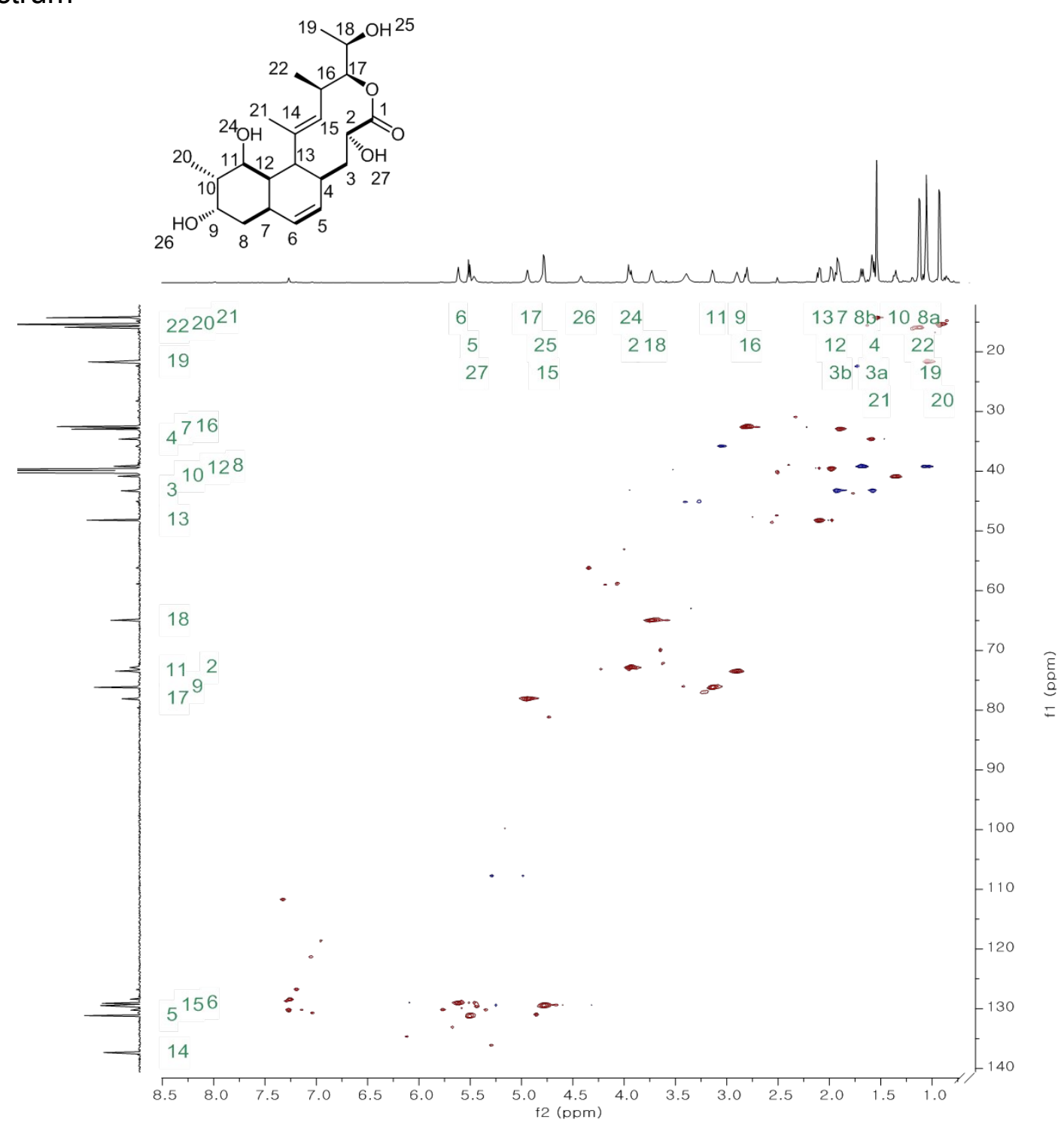


J. HMBC NMR spectrum (refined view)

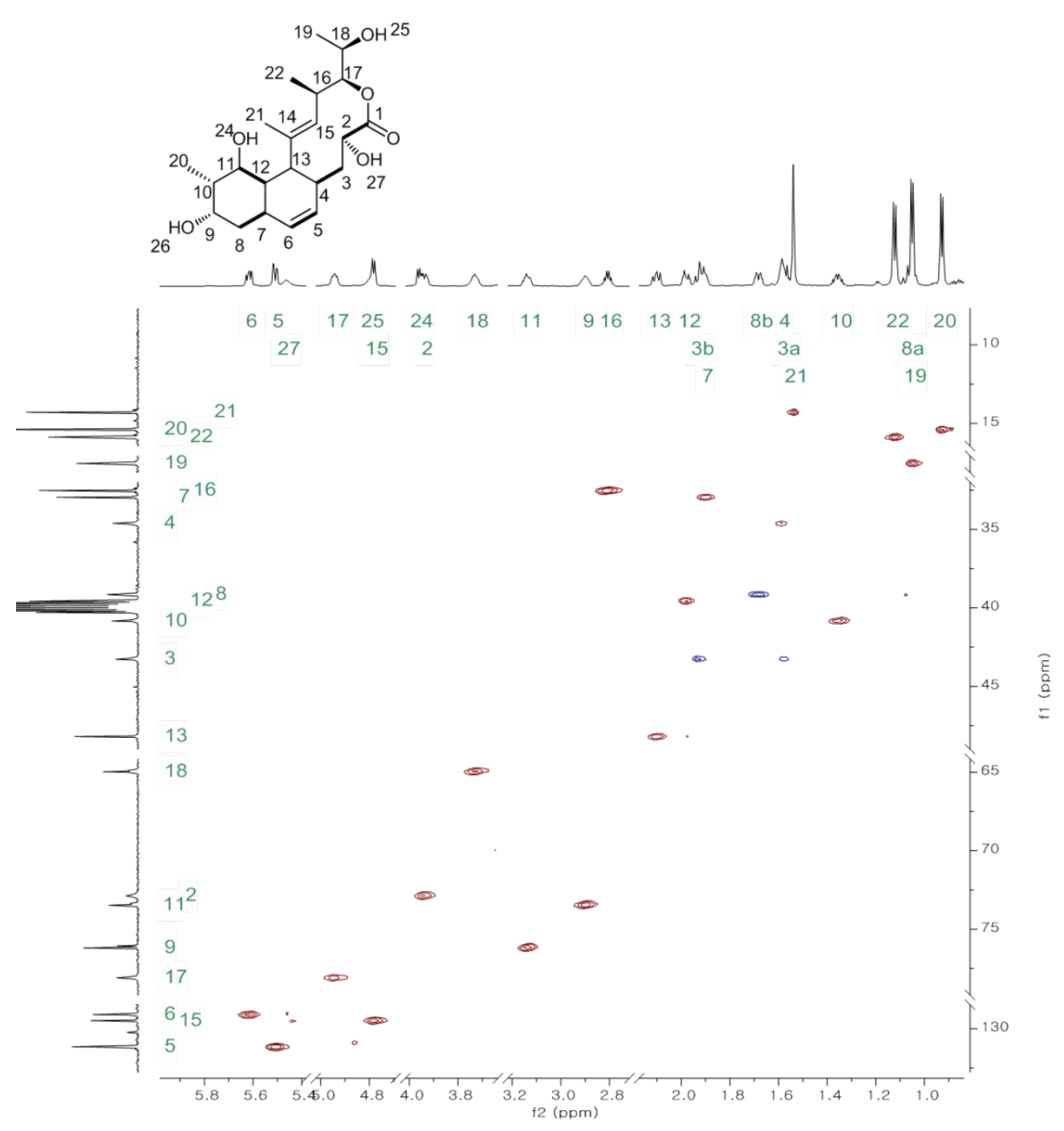


K. NOSEY NMR spectrum

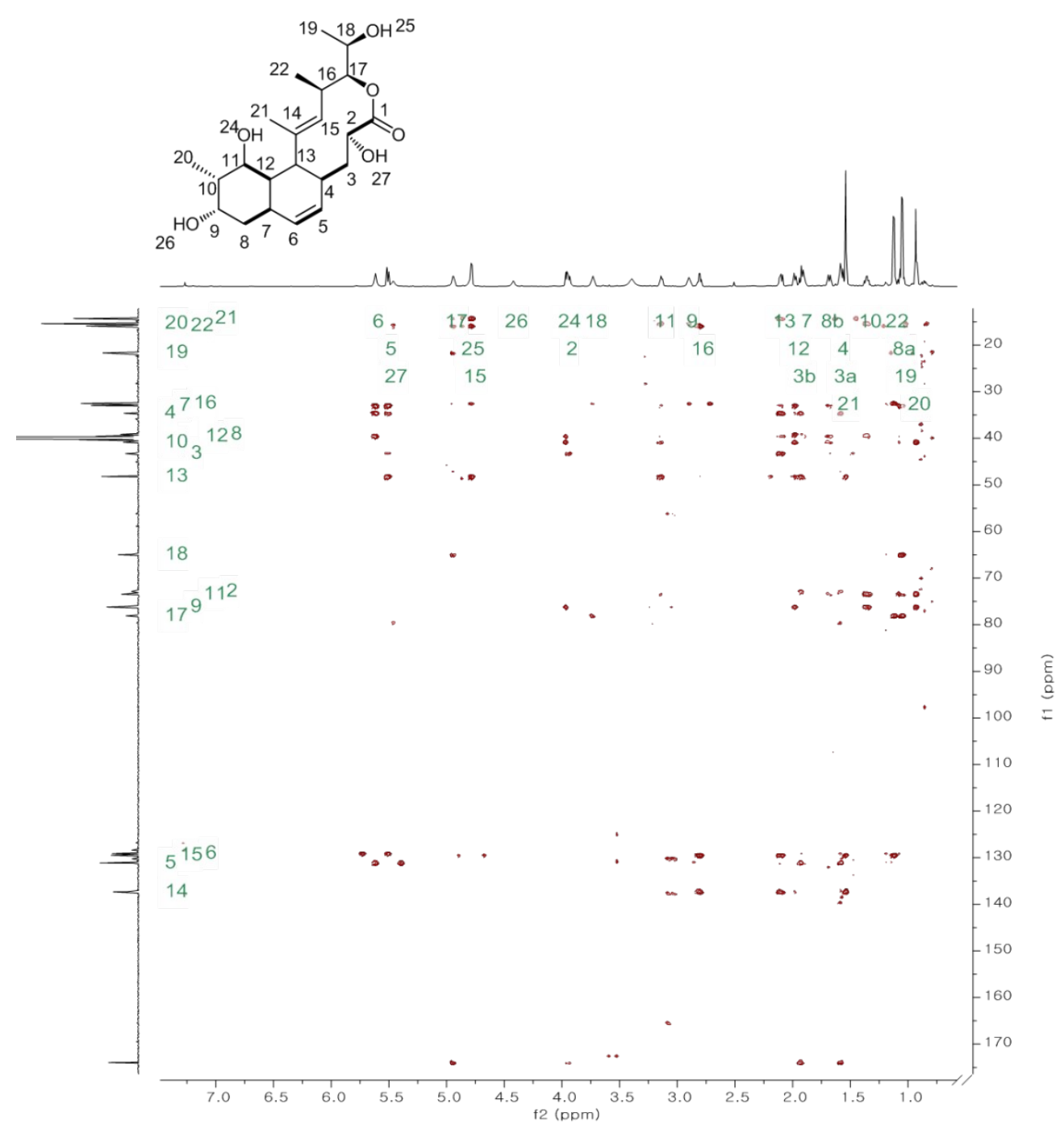


L. NOSEY NMR spectrum (refined view)

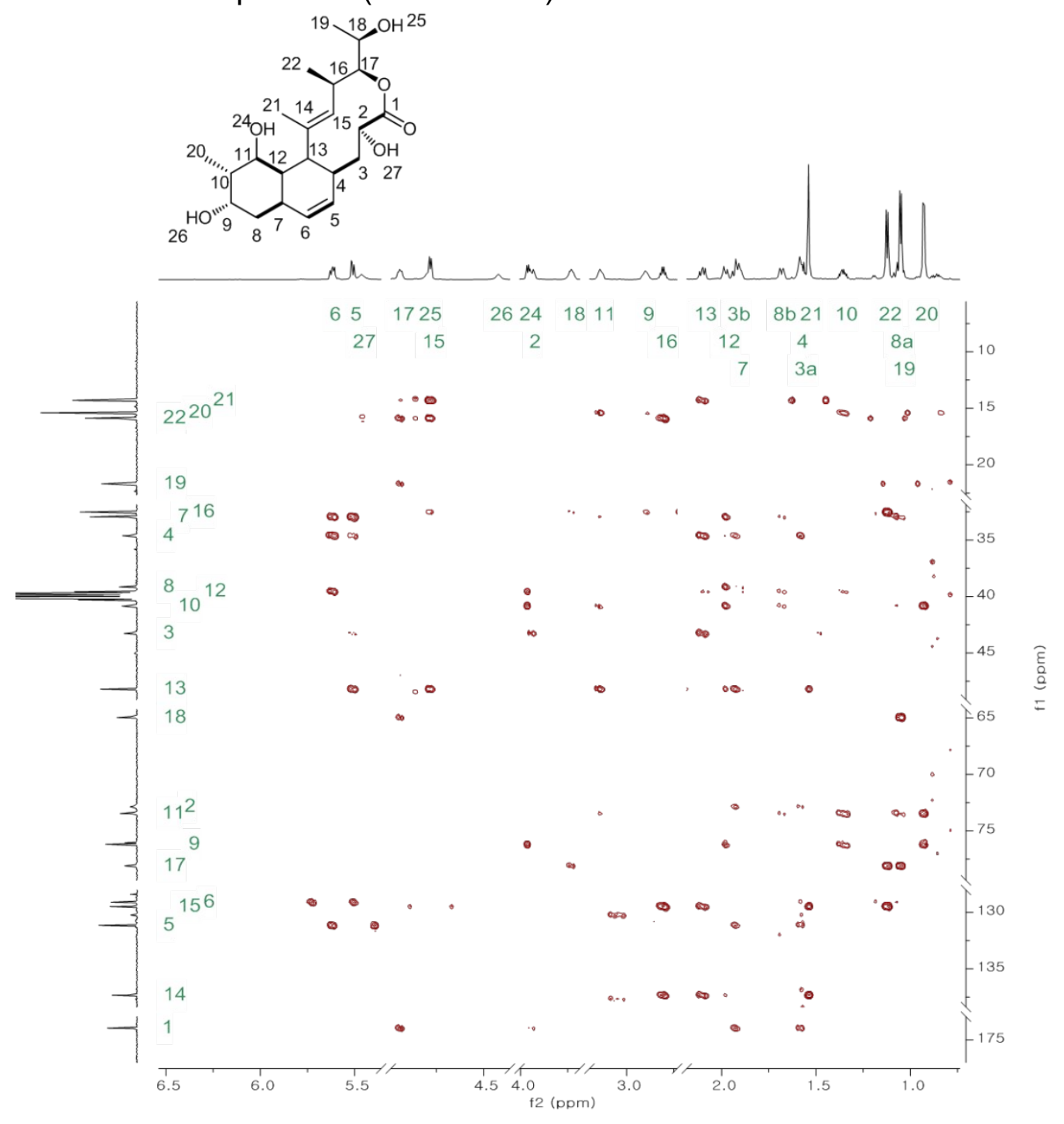


Figure S18. Different NMR spectrum of 23-demethyl-8, 13 deoxynargenicin (9)

\section{A. ${ }^{1} \mathrm{H}$ NMR spectrum of 9 in $700 \mathrm{MHz}$, DMSO-d6}

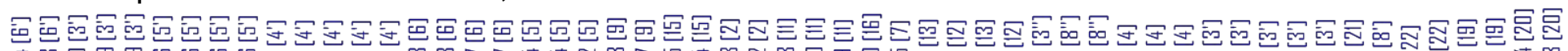

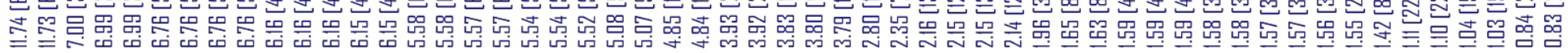

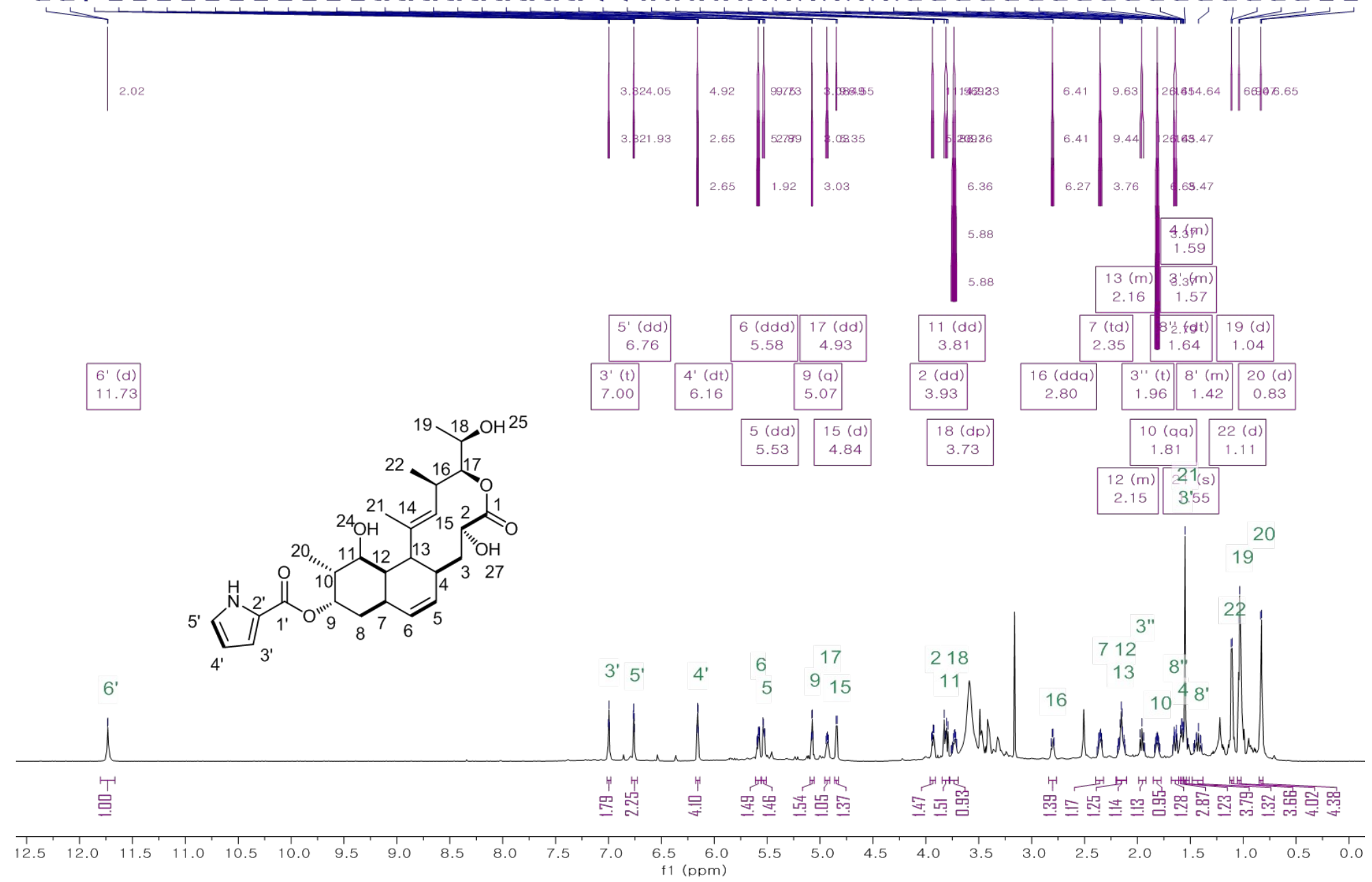


B. ${ }^{13} \mathrm{C}$ NMR spectrum of 9 in $176 \mathrm{MHz}$, DMSO-d6
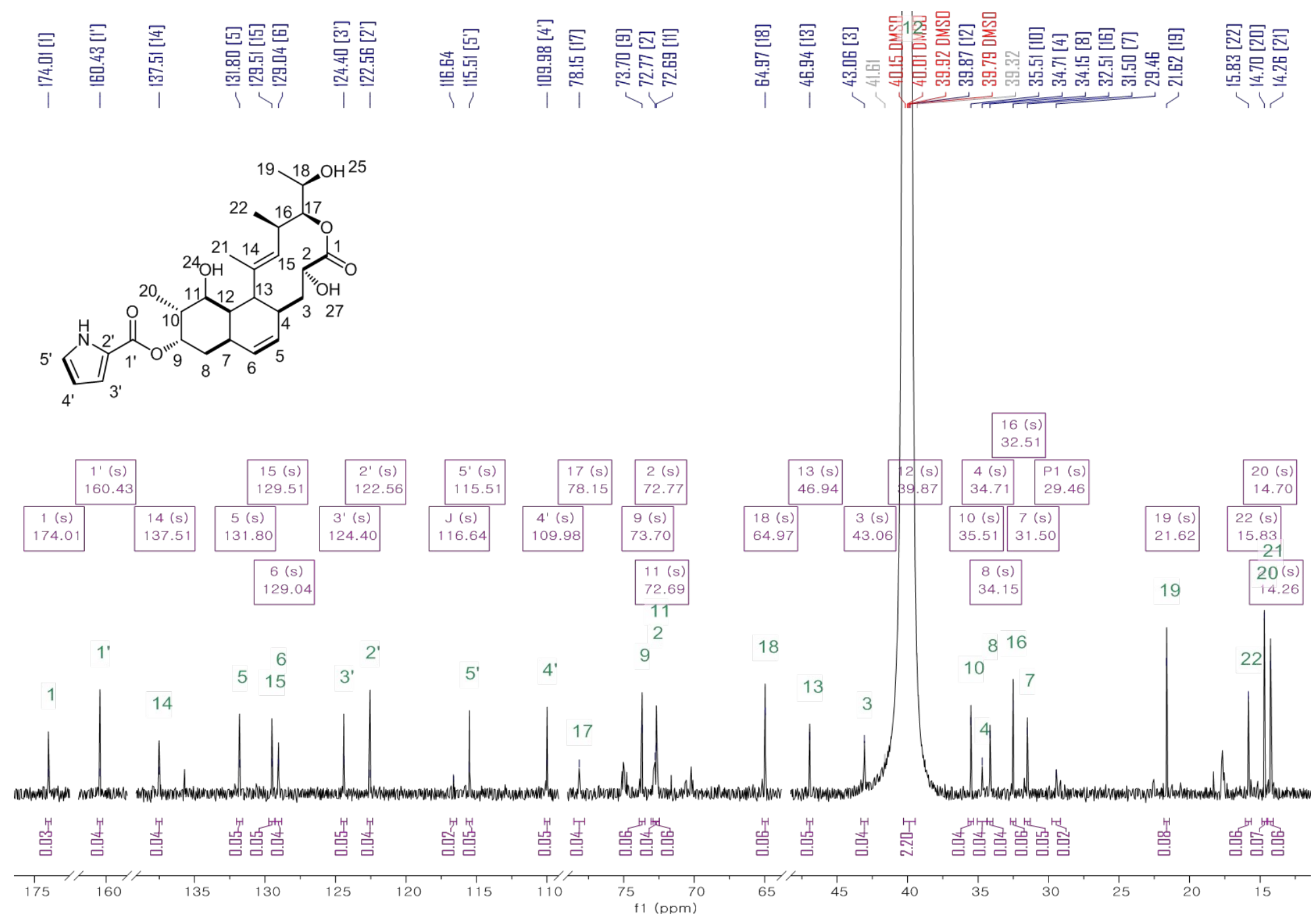
C. COSY NMR spectrum

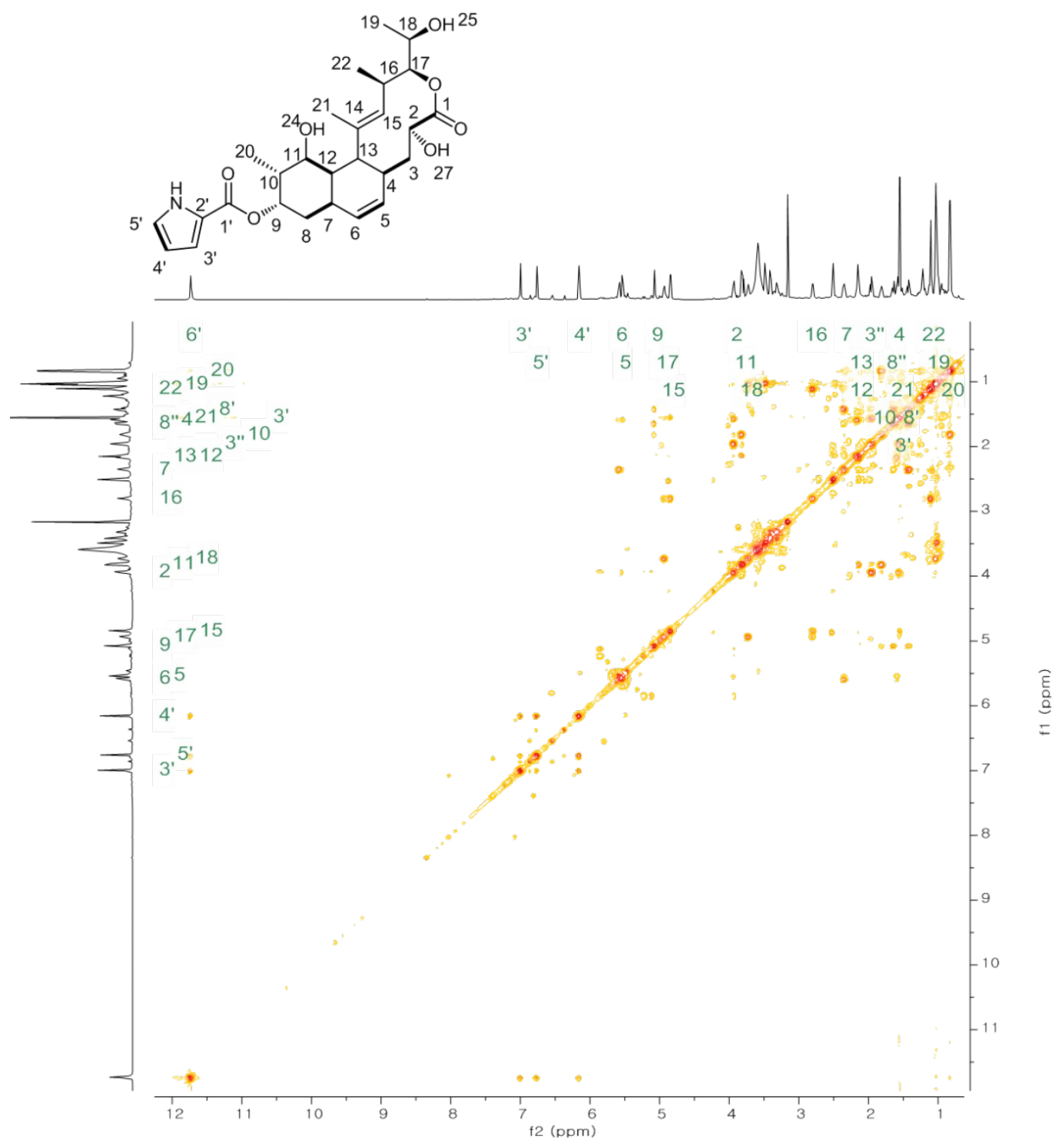


D. COSY NMR spectrum (refined)

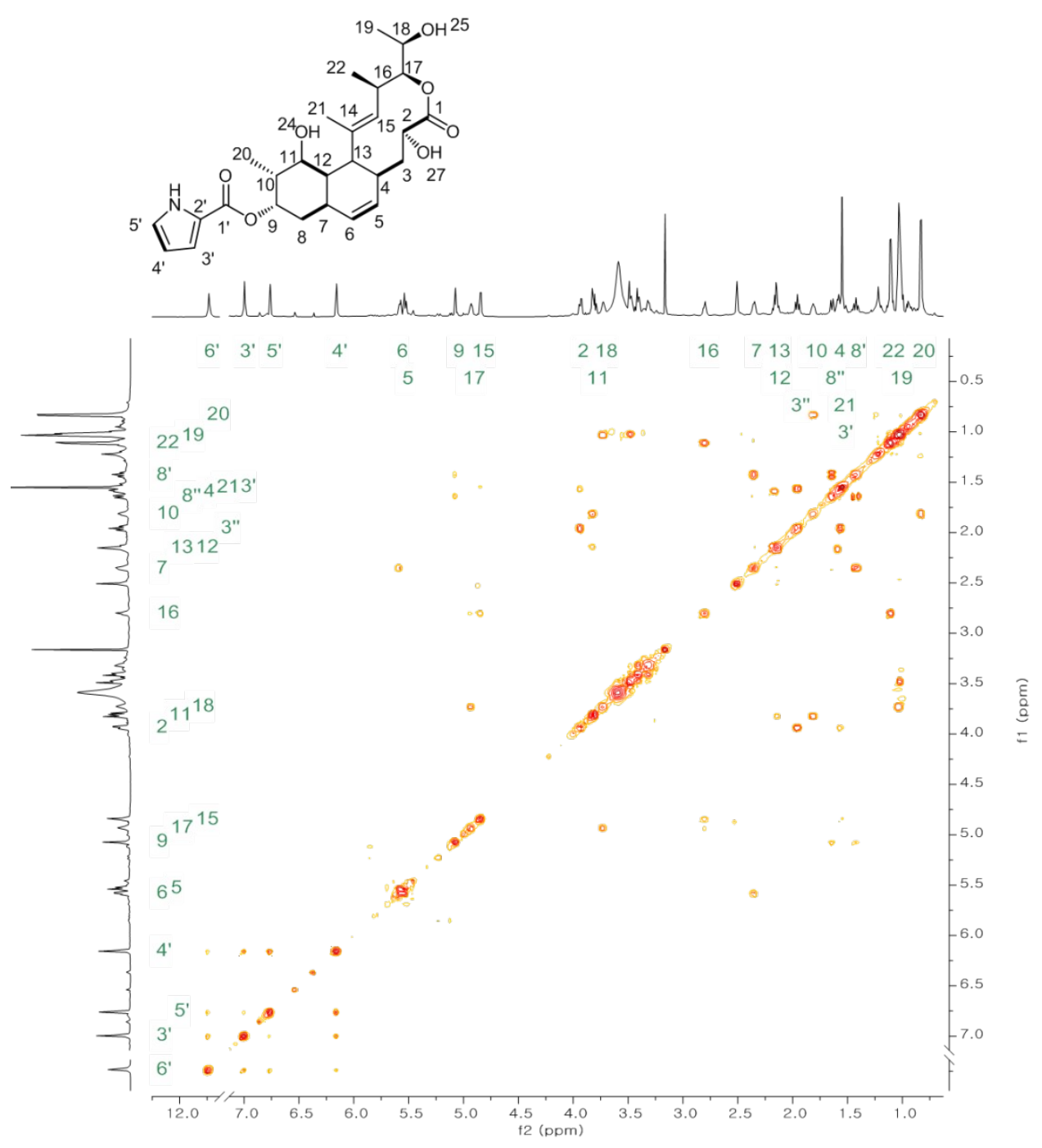


E. HSQC-DEPT NMR spectrum

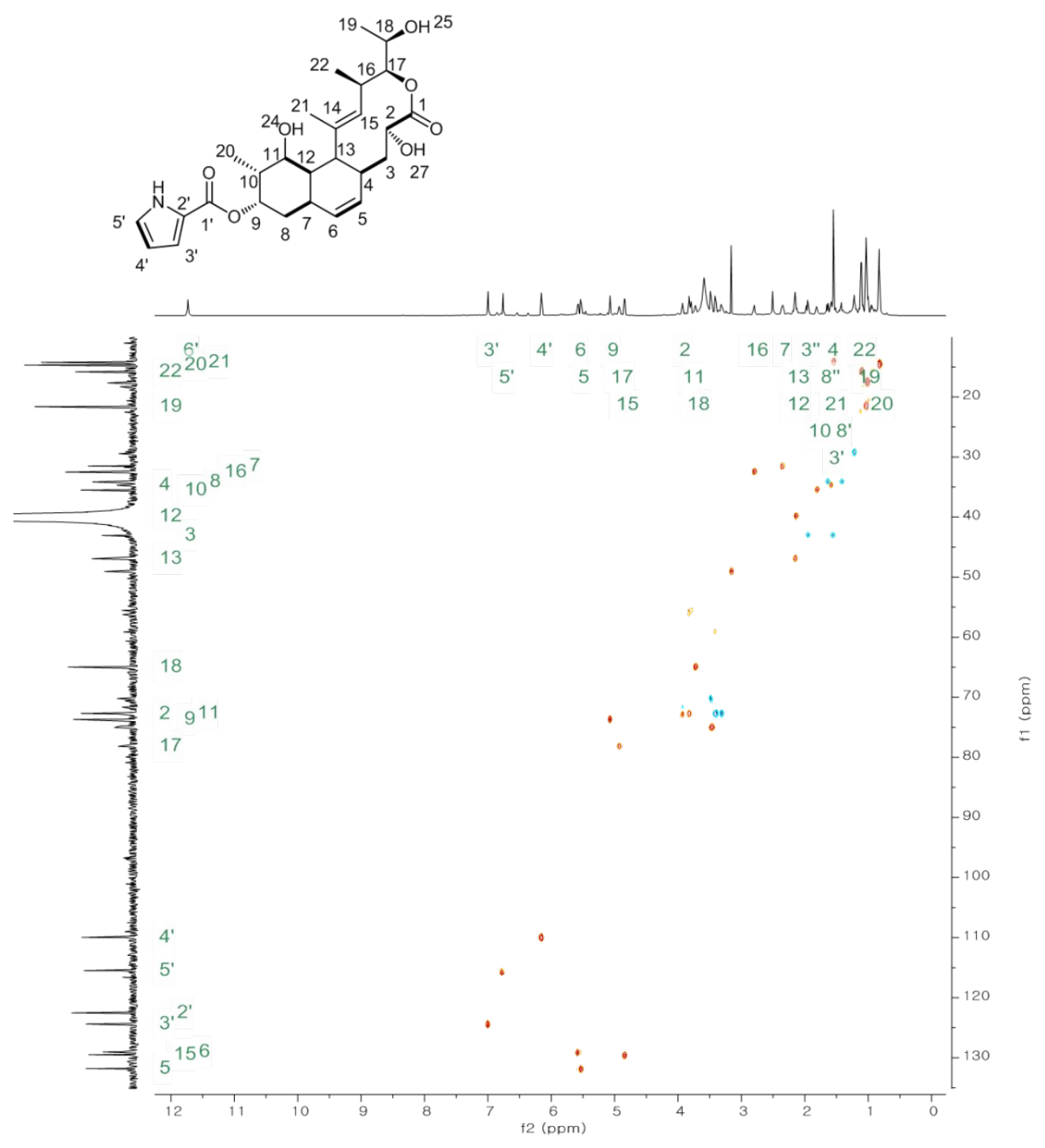




\section{F. HMBC NMR spectrum}

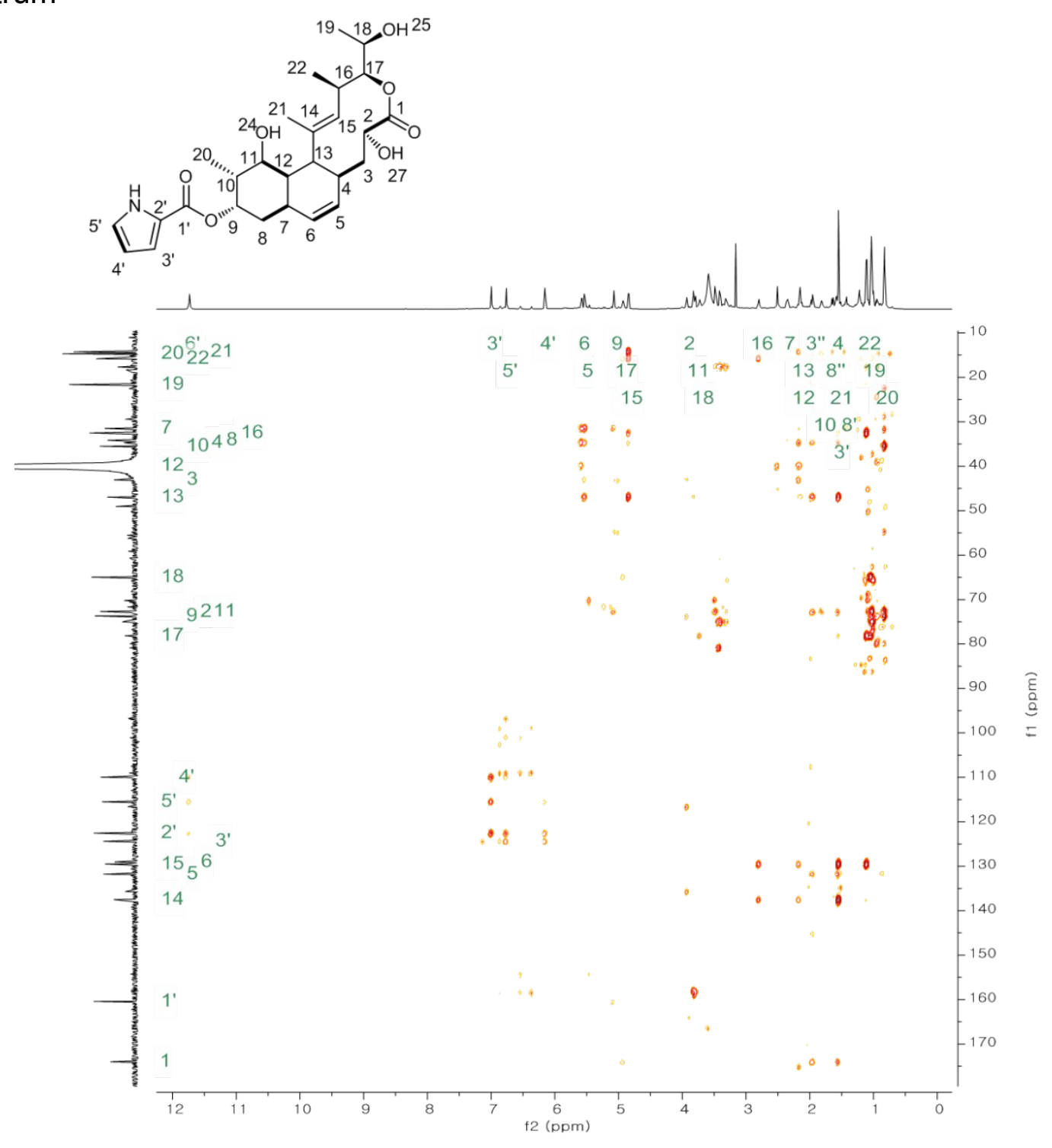


G. ROSEY NMR spectrum

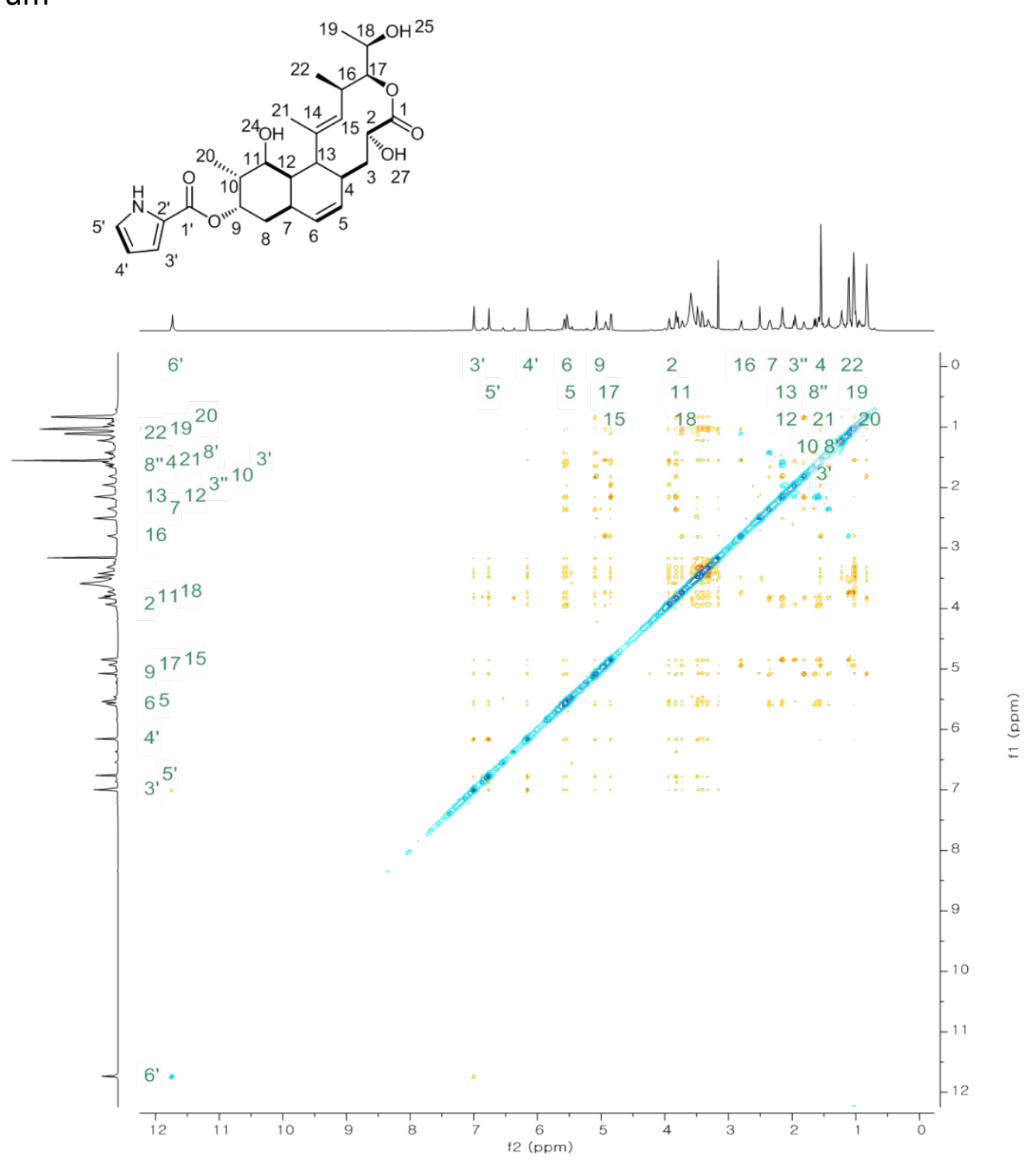


H. ROSEY NMR spectrum (refined view)

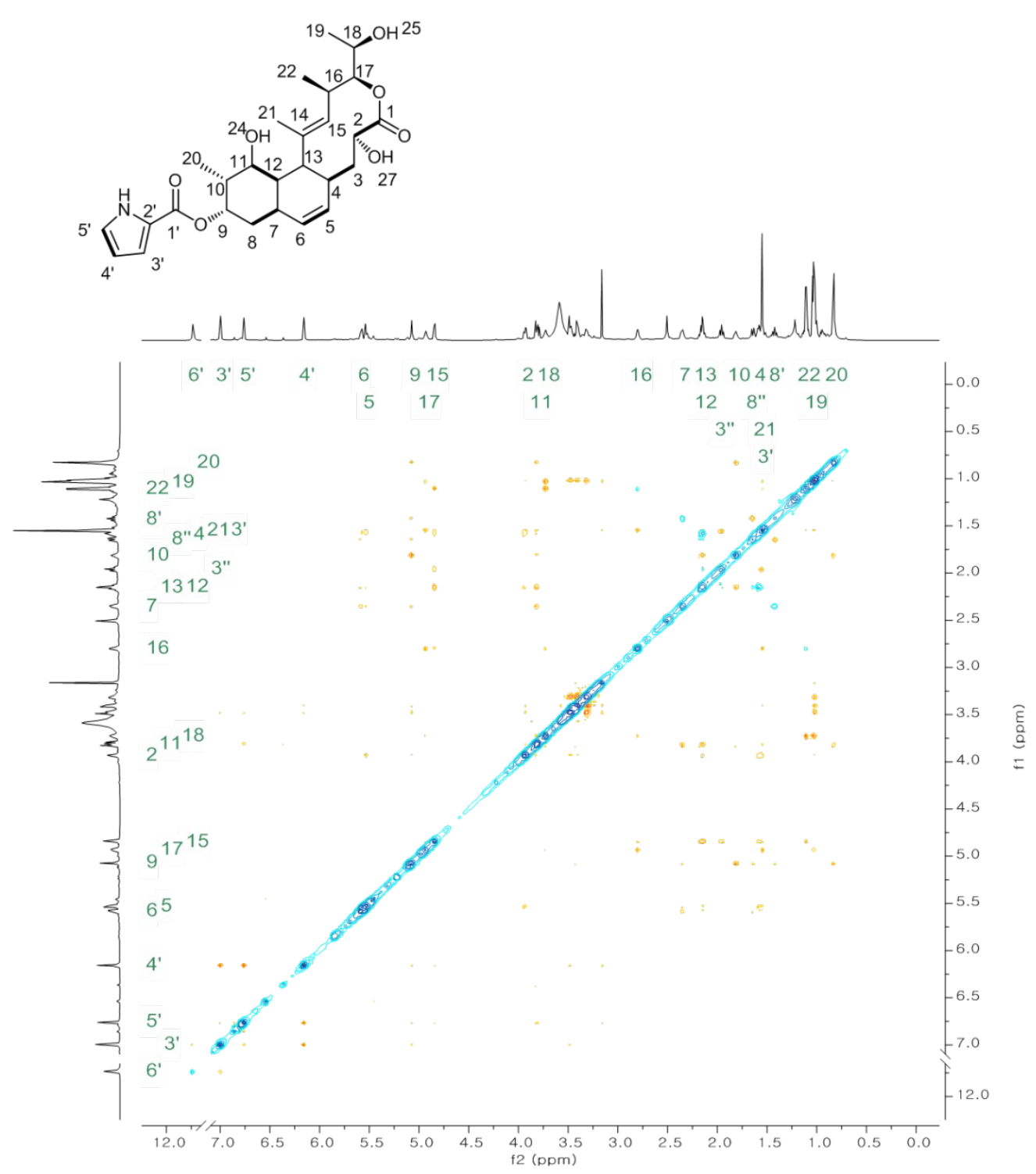


I. HSQC-HMBC spectra overlap. Red cross peaks are HSQC correlation, green are HMBC correlation

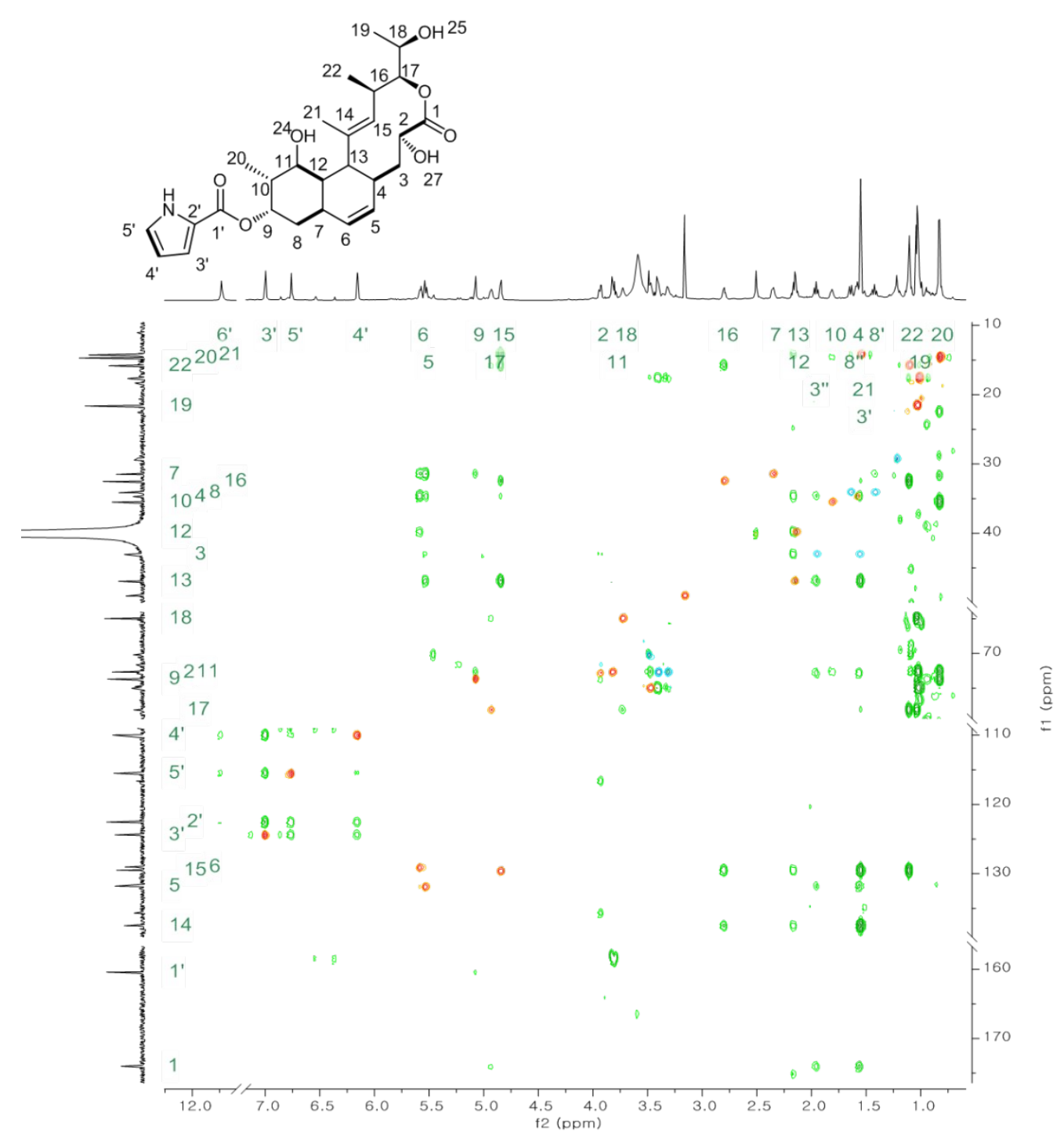


Figure S19. SDS Page of purified proteins (a) NgnM and (b) NgnO3

a

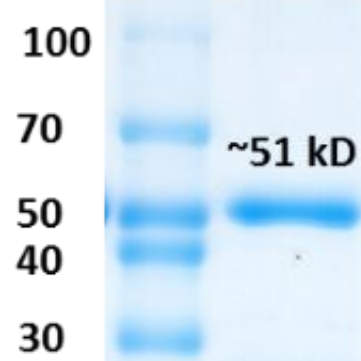

20

15 b

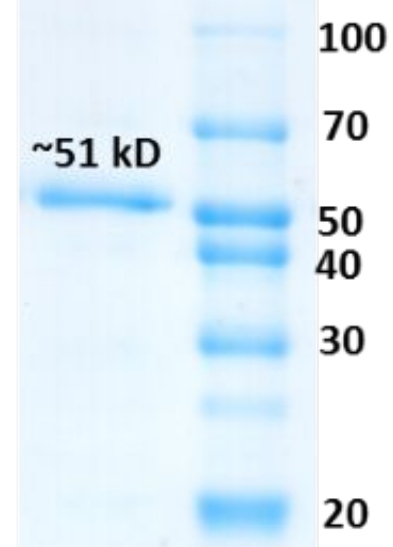

15 
Figure S20. Reaction of different substrates with NgnM (a) Enzymatic reaction of 8 with NgnM, (b) Control reaction with boiled enzyme , (c) Purified standard (8); (d) Enzymatic reaction of 9 with NgnM, (e) Control reaction with boiled enzyme , (f) Purified standard (9)
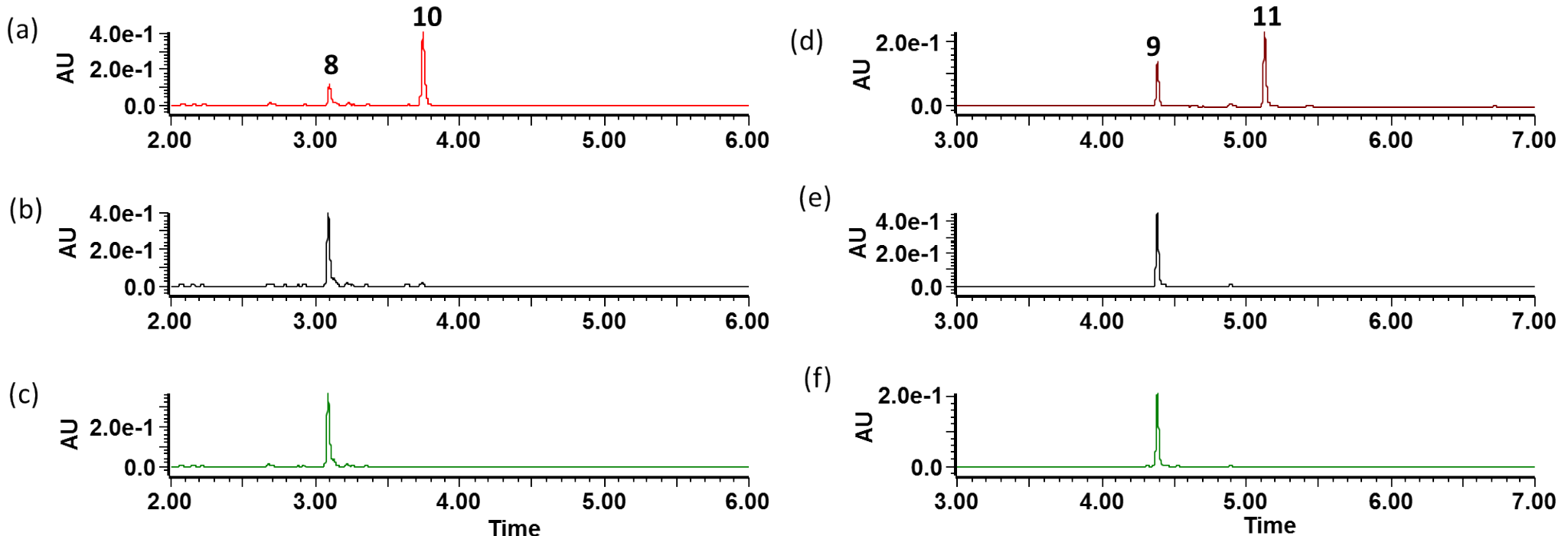
Figure S21. HR-QTOF Mass chromatogram of different compounds generated by enzyme reaction with NgnM (a) Mass chromatogram for 8,13 deoxynodusmicin (10) and (b) 8, 13 deoxynargenicin (11). UV absorbance of different compounds (c) 10 and (d) 11
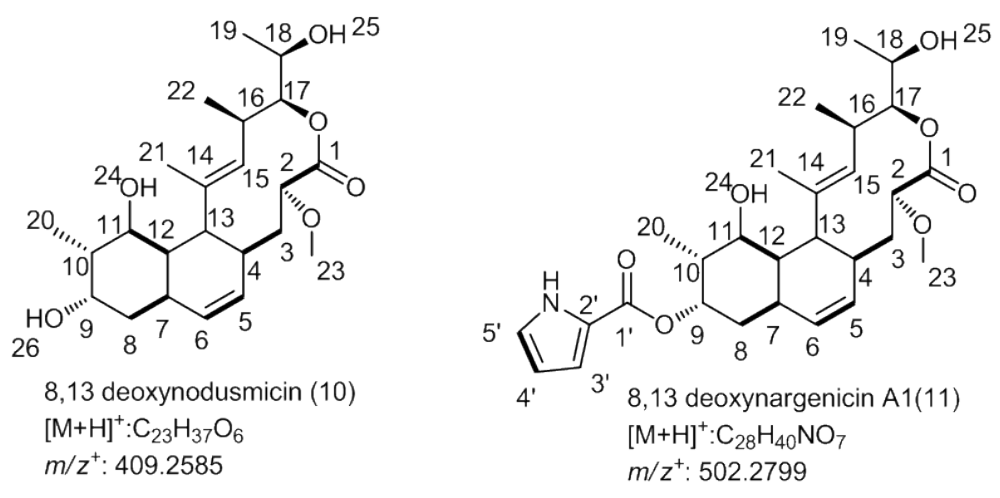

(a)

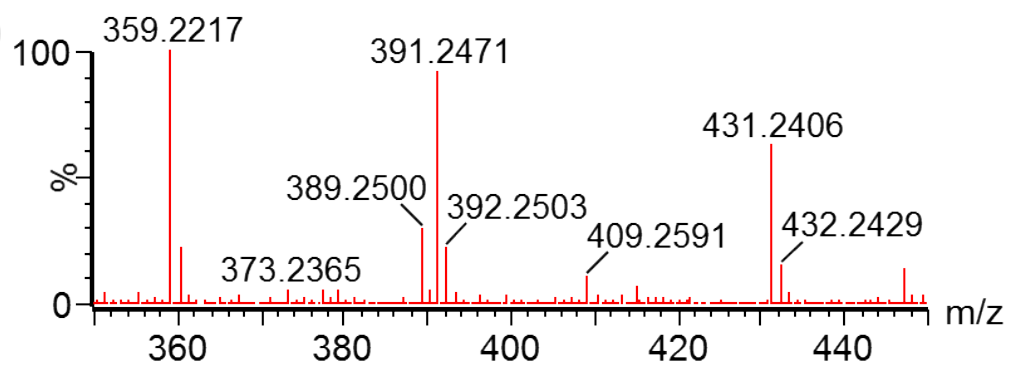

(b)

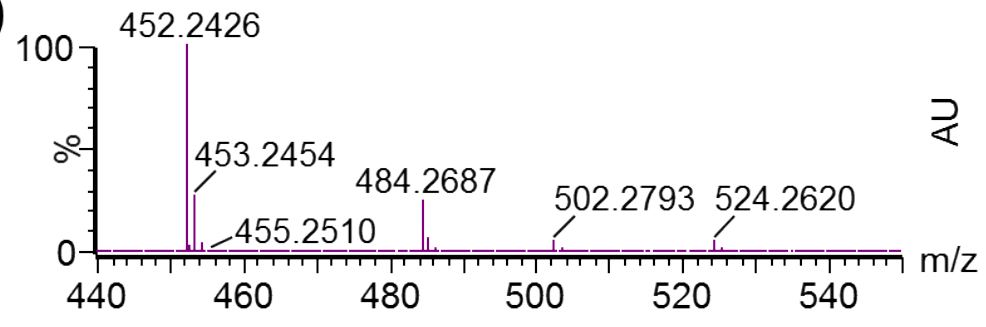

(c)

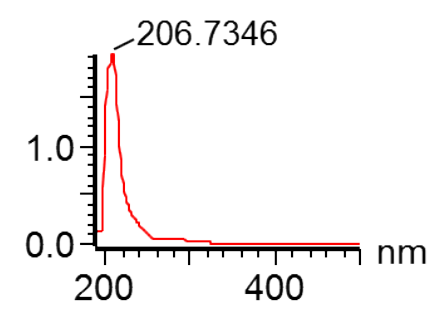

(d)

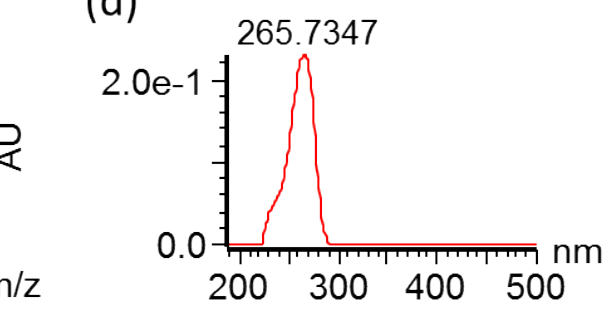


Figure S22. Different NMR spectrum of 8, 13 deoxynodusmicin (10)

A. ${ }^{1} \mathrm{H}$ NMR spectrum of 10 in $700 \mathrm{MHz}$, DMSO-d6

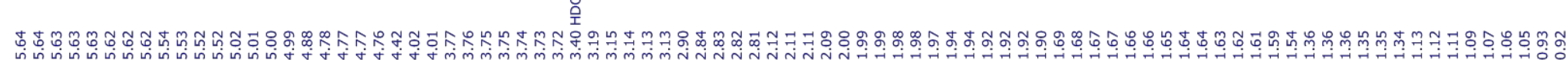

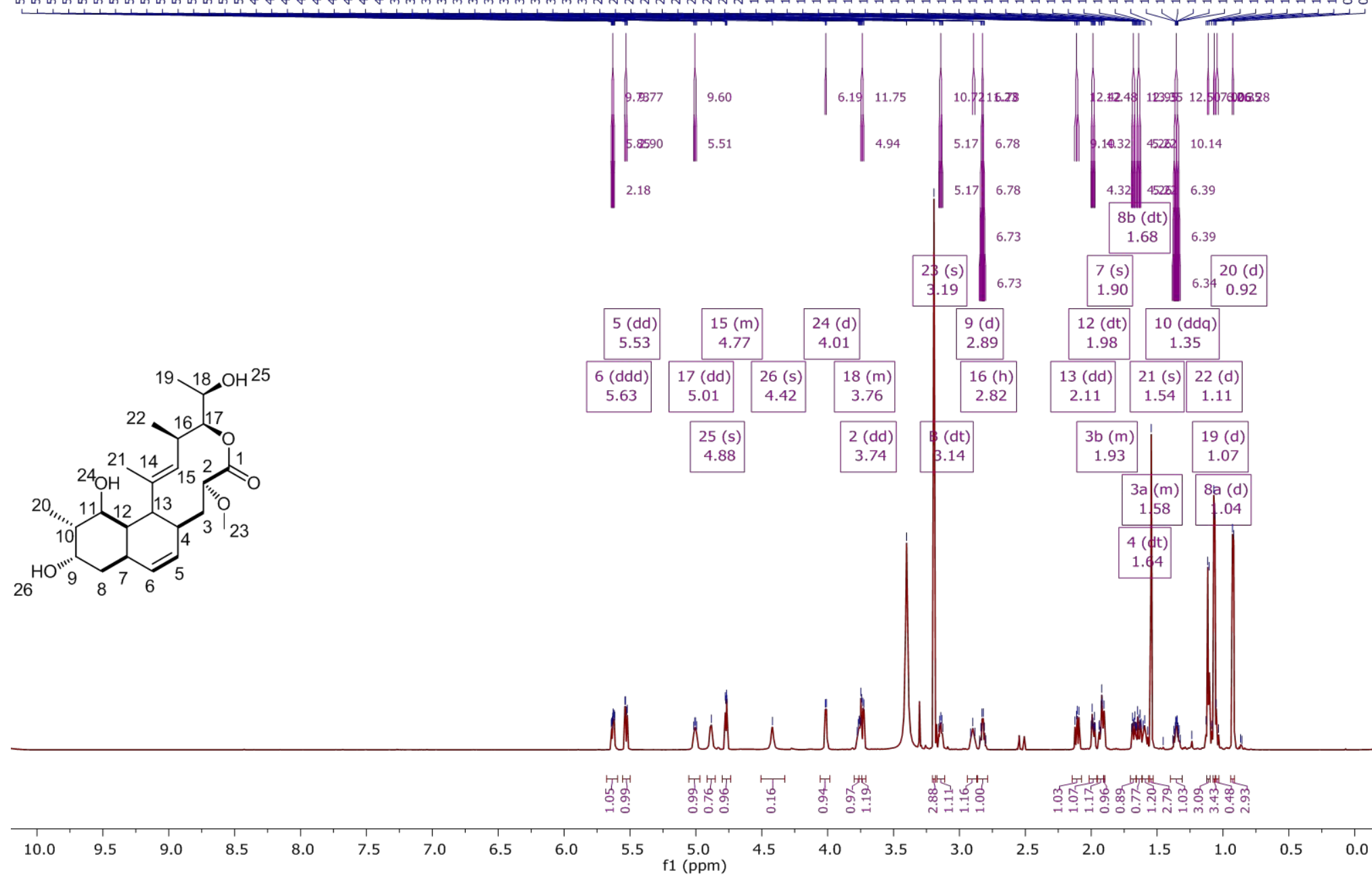


B. ${ }^{1} \mathrm{H}$ NMR spectrum of 10 in $700 \mathrm{MHz}$, DMSO-d6 (refined view)

오

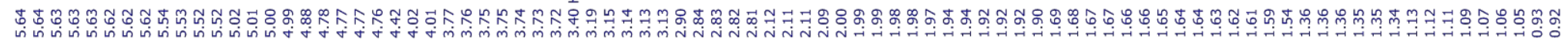

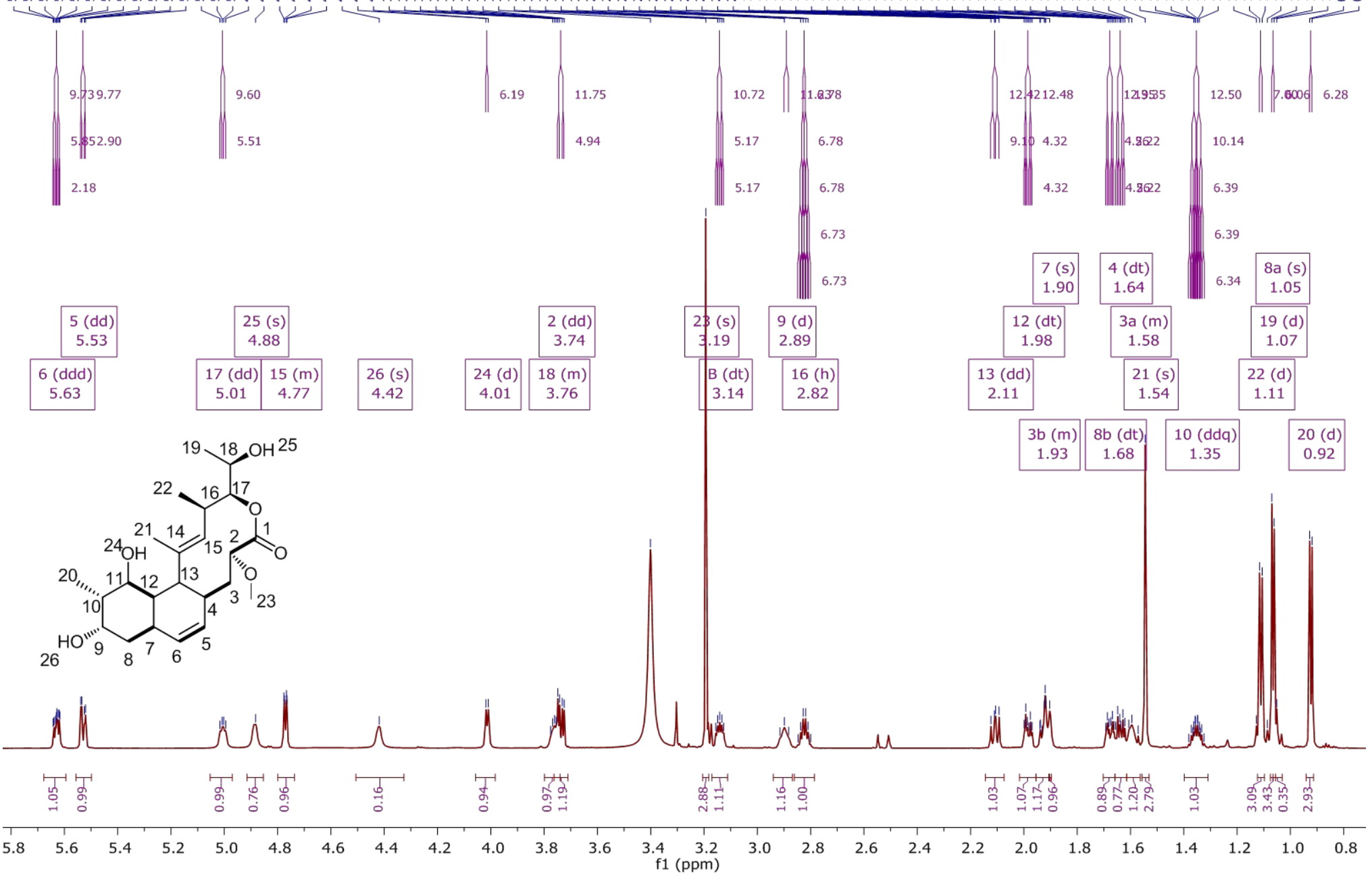


C. ${ }^{13} \mathrm{C}$ NMR spectrum of 10 in $176 \mathrm{MHz}$, DMSO-d6

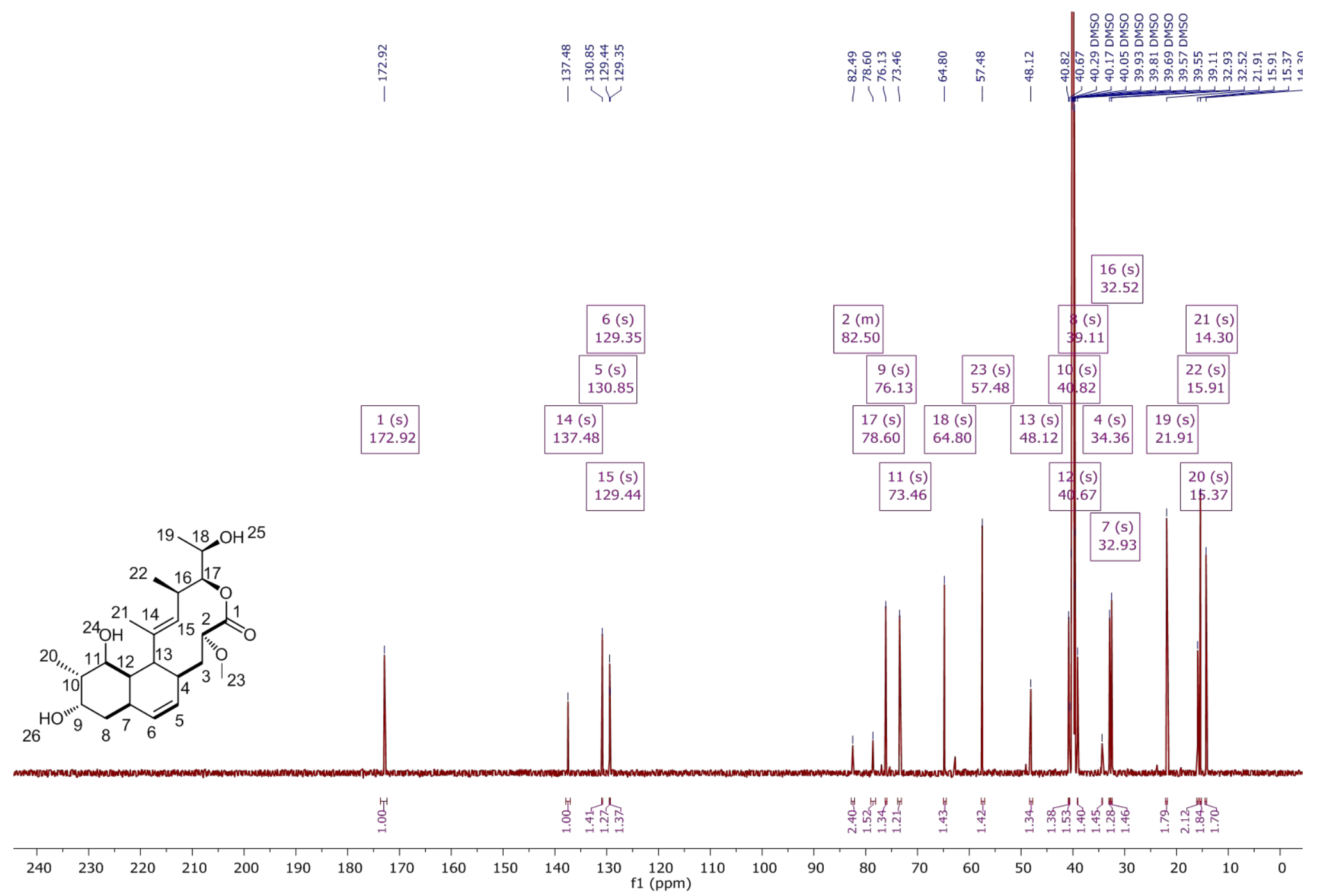


D. ${ }^{13} \mathrm{C}$ NMR spectrum of 10 in $176 \mathrm{MHz}$, DMSO-d6 (refined view)

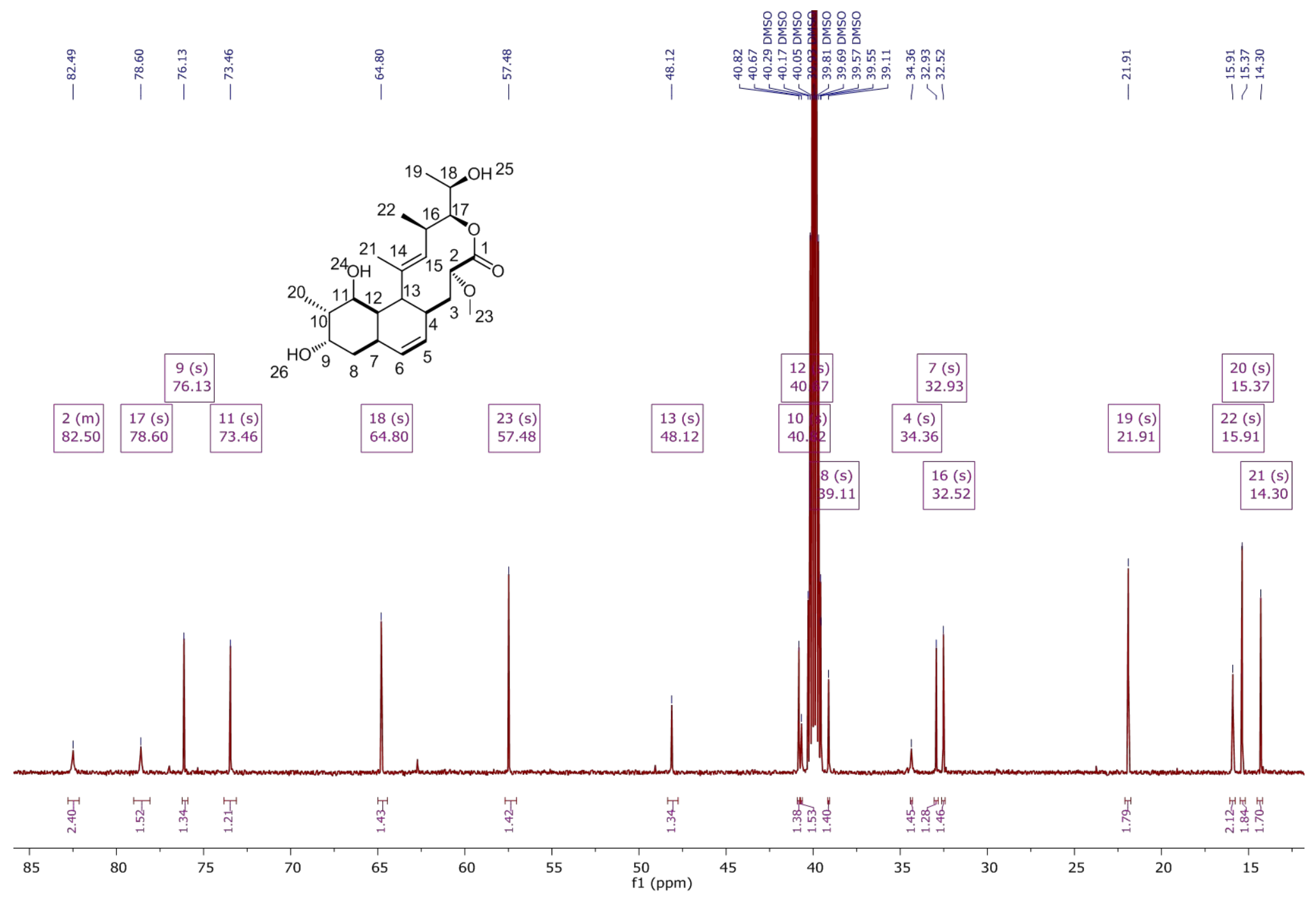


Figure S23. Different NMR spectrum of 8,13 deoxynargenicin (11)

A. ${ }^{1} \mathrm{H}$ NMR spectrum of 11 in $700 \mathrm{Mhz}$, DMSO-d6

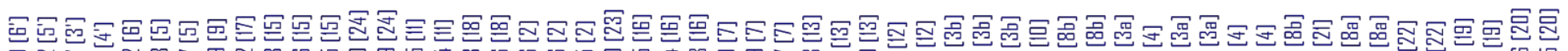

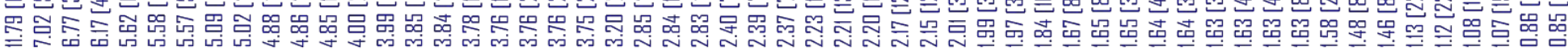
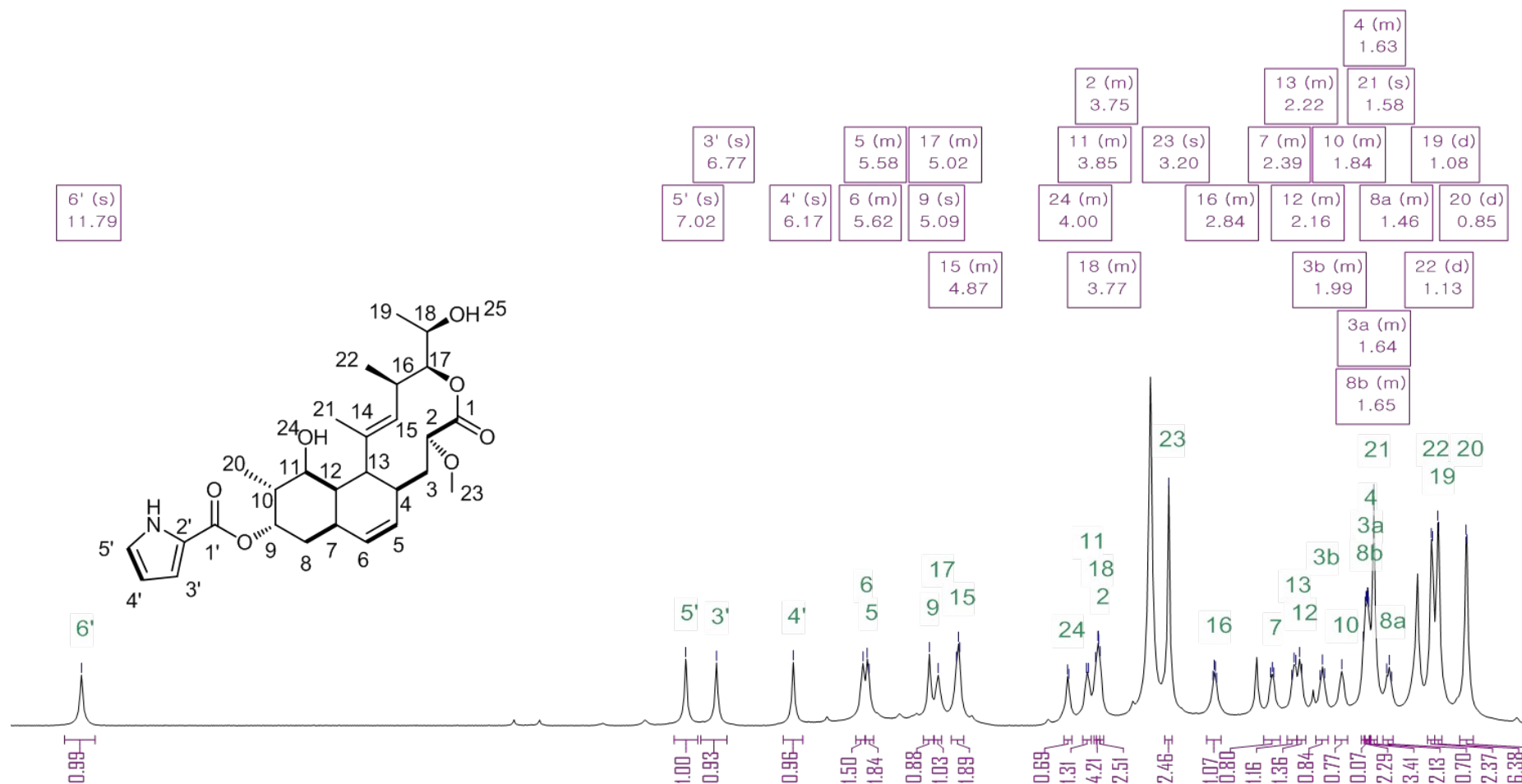

19

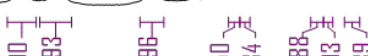

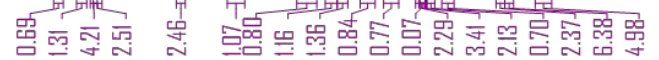

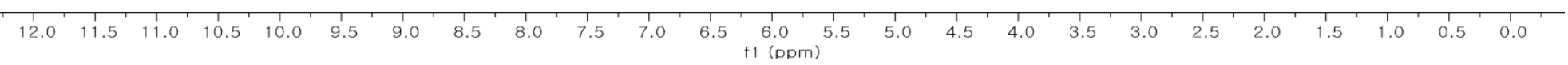


B. ${ }^{1} \mathrm{H}$ NMR spectrum of 11 in $700 \mathrm{Mhz}$, DMSO-d6 (refined view)

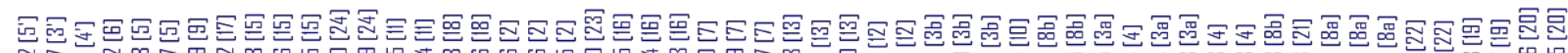

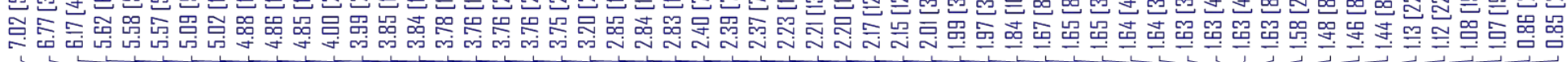
(2)
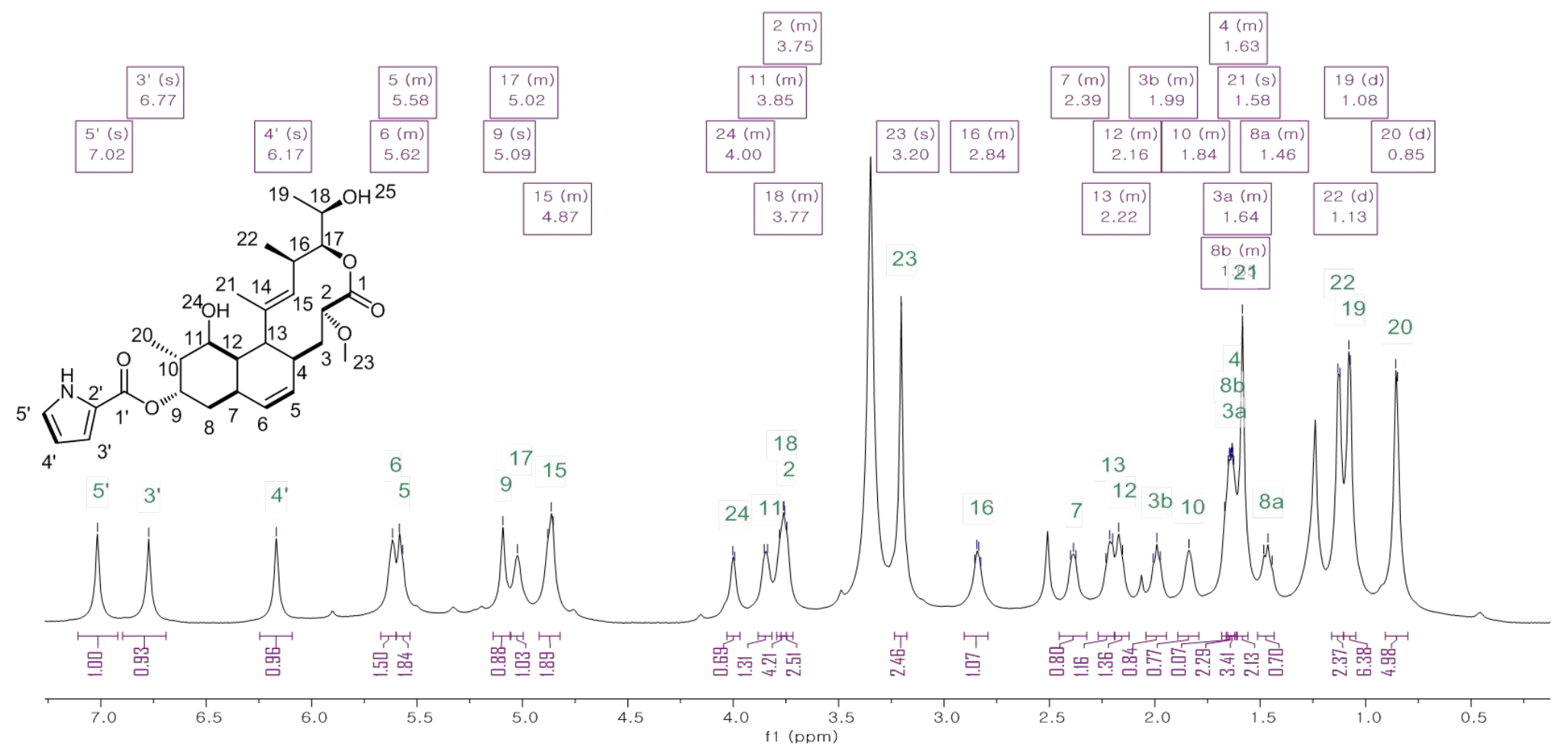
C. ${ }^{13} \mathrm{C}$ NMR spectrum of 11 in $176 \mathrm{~Hz}$, DMSO-d6

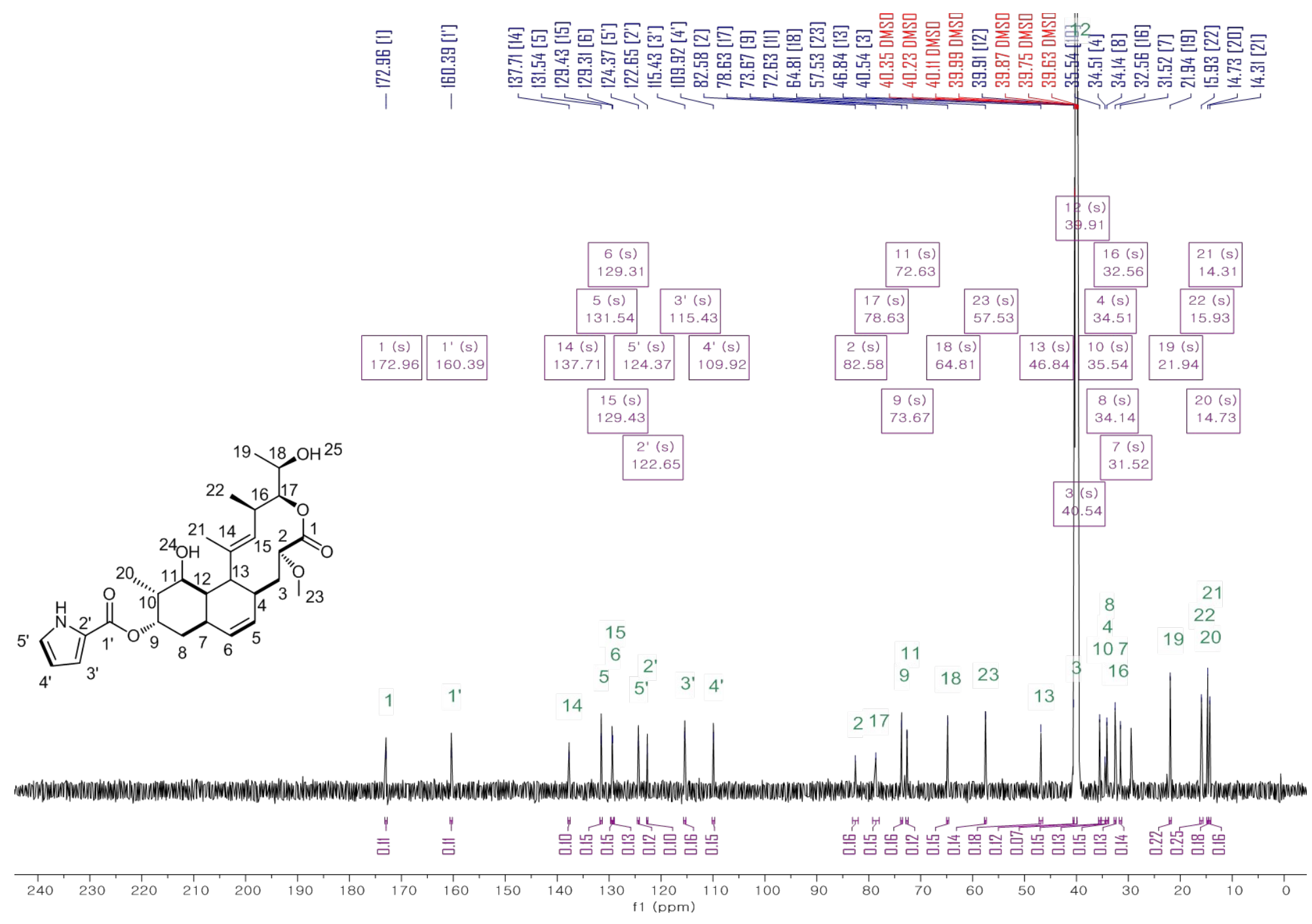


D. COSY NMR spectrum

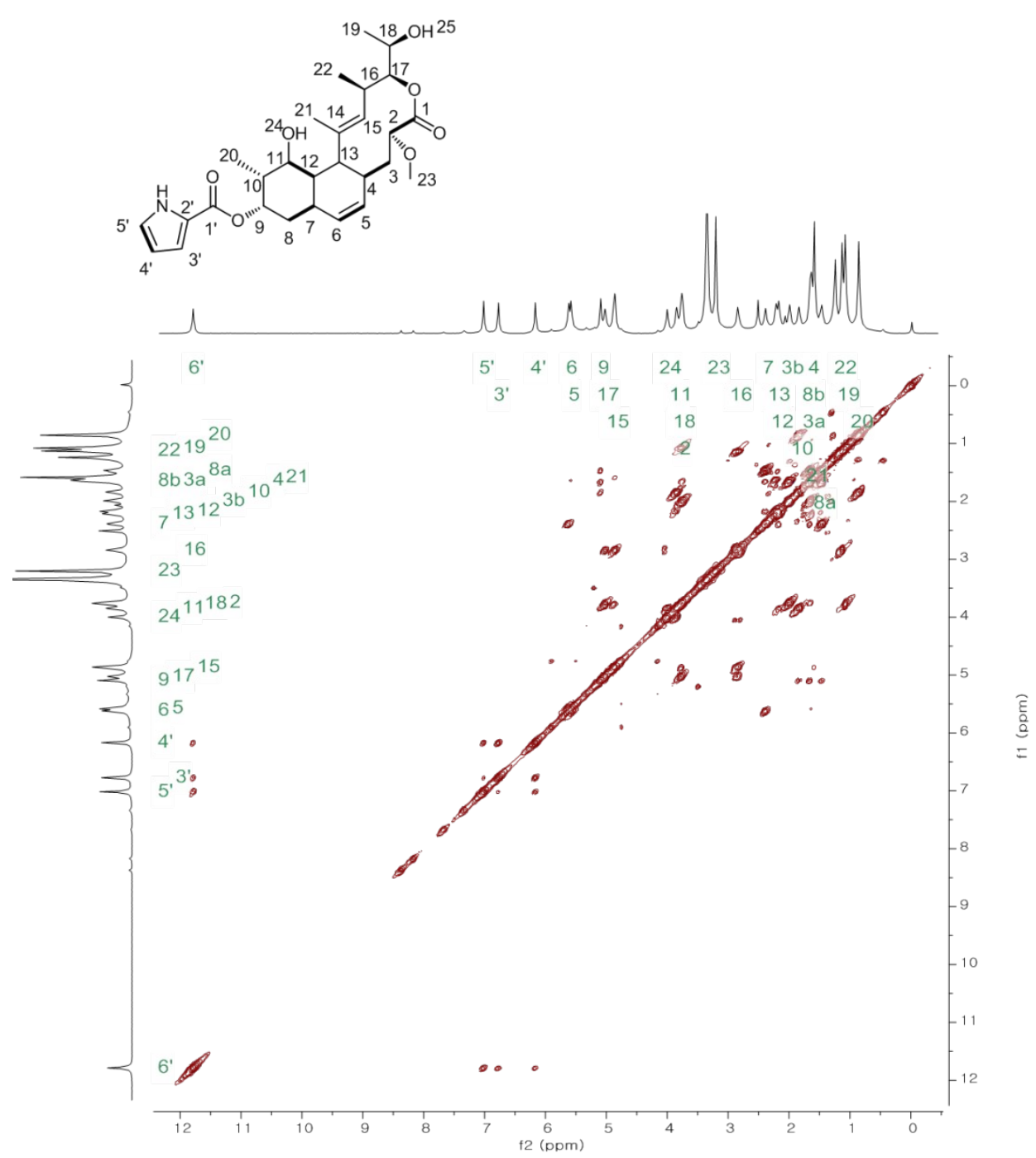


E. HSQC-DEPT NMR spectrum

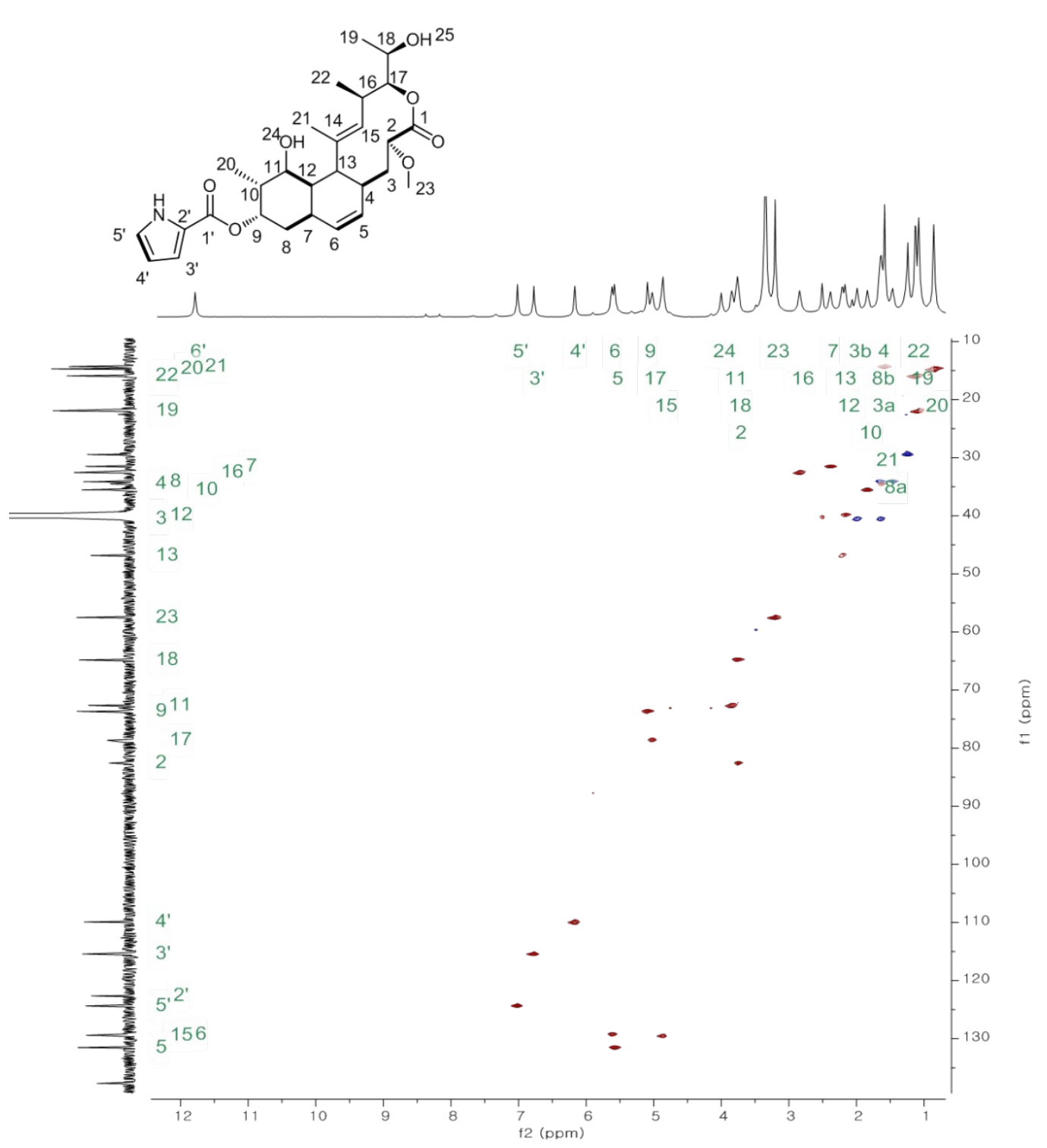


F. HMBC NMR spectrum

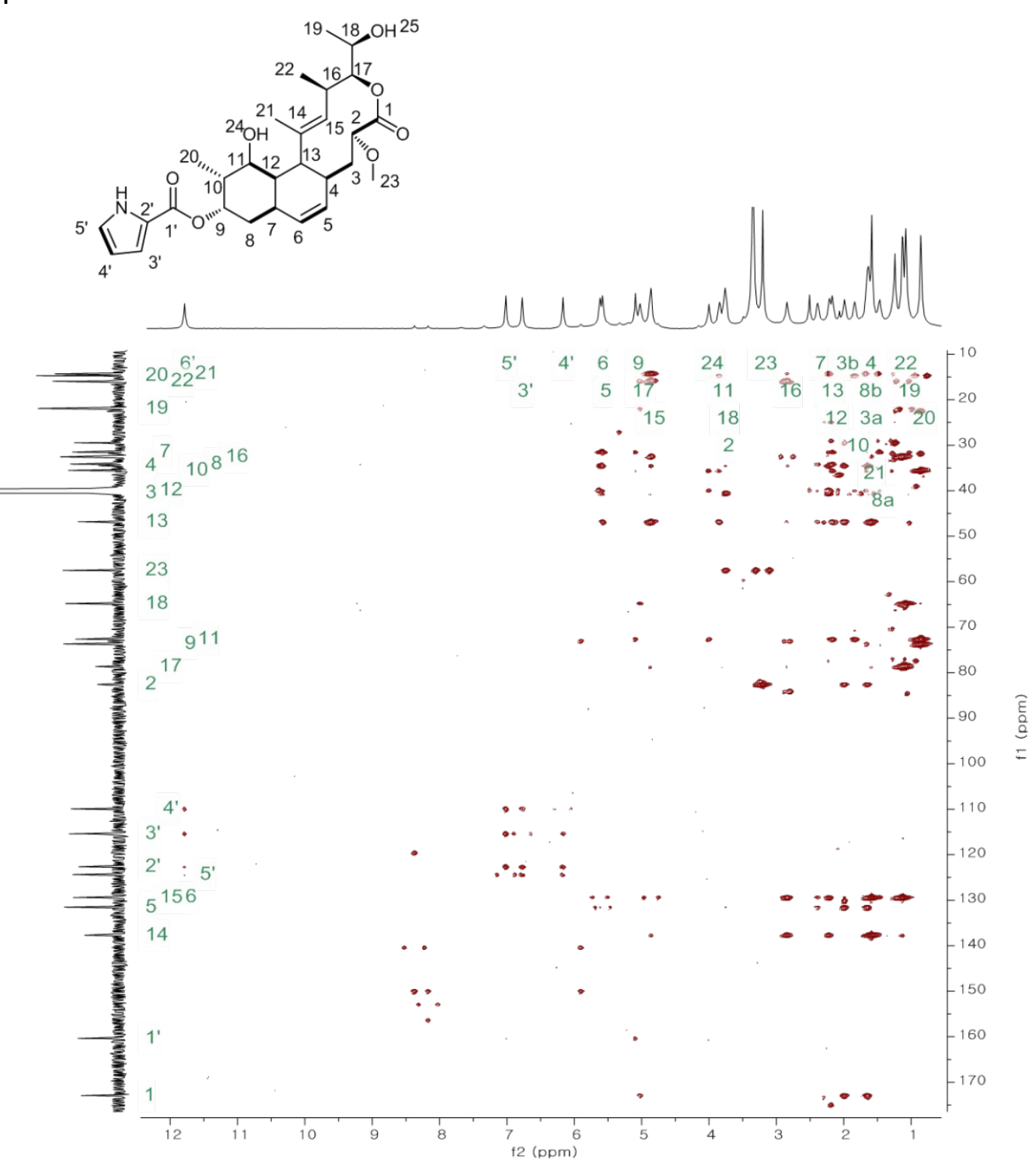


G. ROSEY NMR spectrum

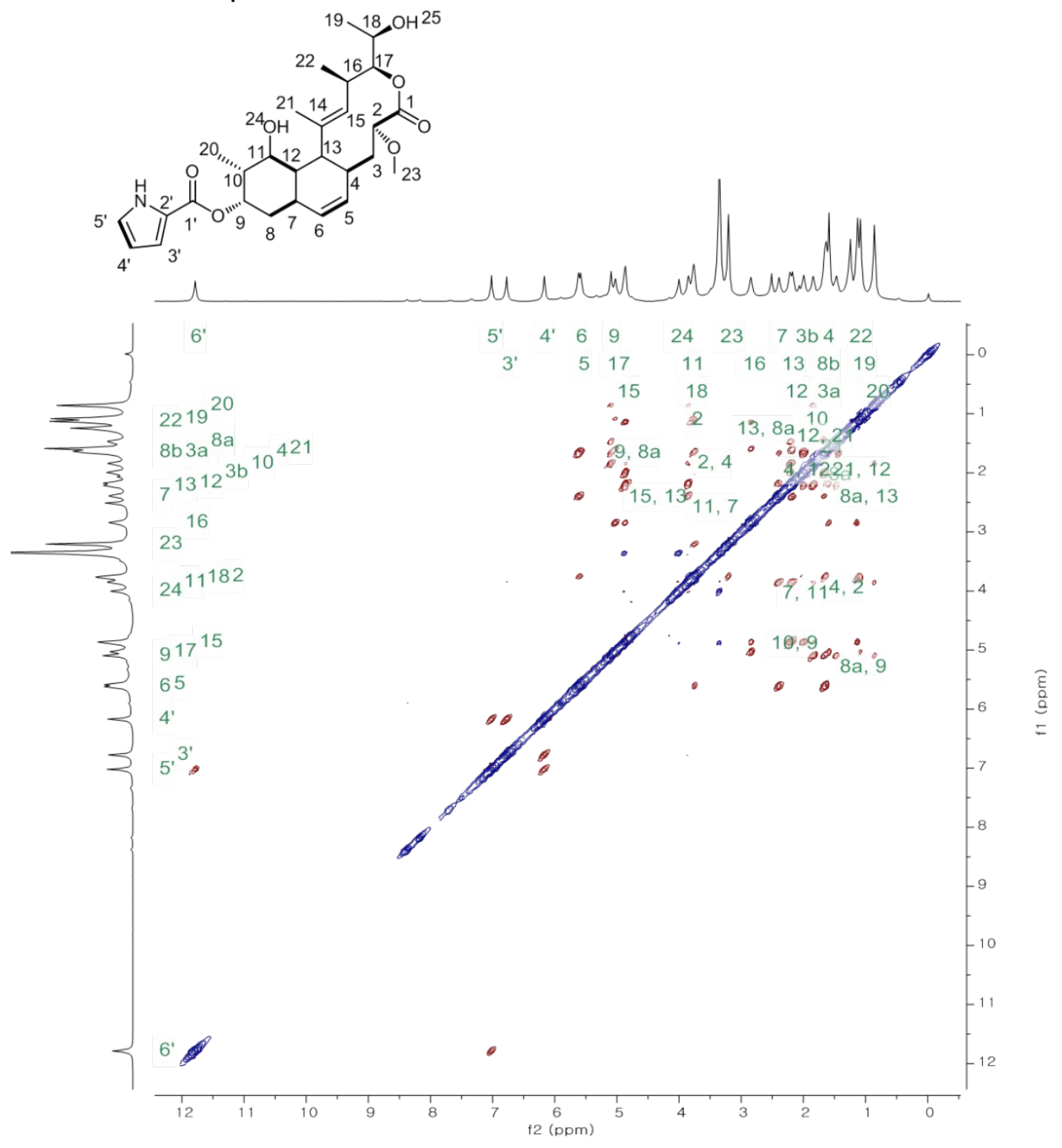


Figure S24. Reaction of different substrates with $\mathrm{NgnO} 3$ (a) Enzymatic reaction of 10 with $\mathrm{NgnO} 3$ which led to $100 \%$ conversion to 2, (b) Purified standard (10); (c) Enzymatic reaction of 11 with NgnO3, (d) Purified standard (11)

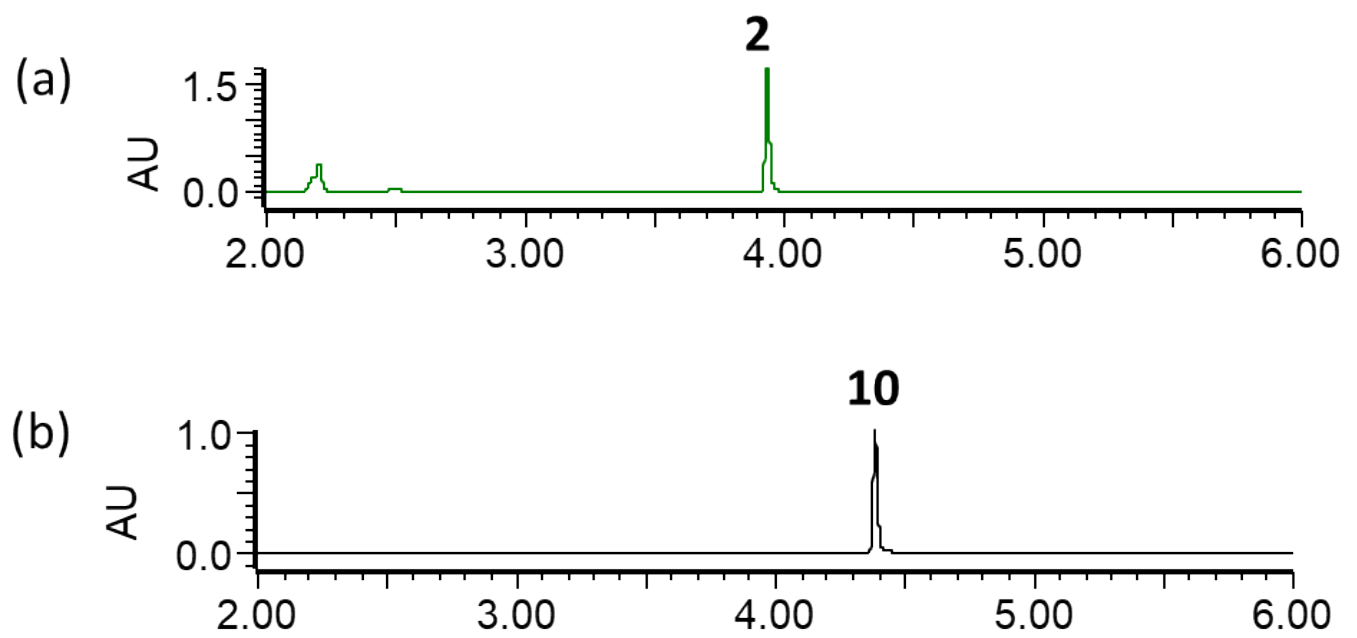

(c)

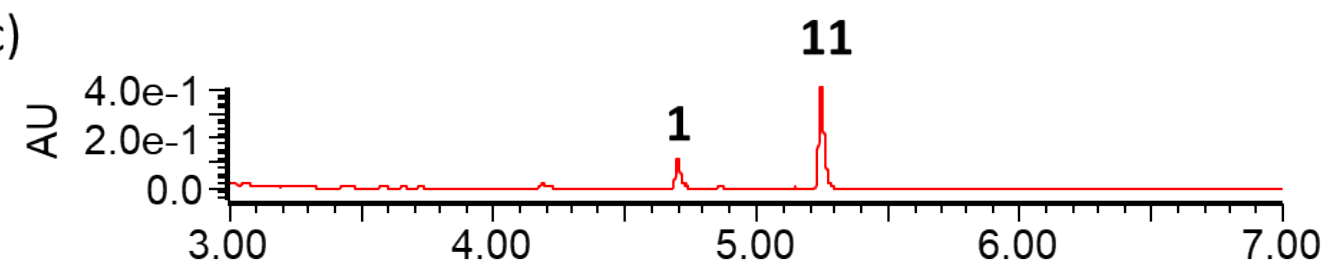

(d)

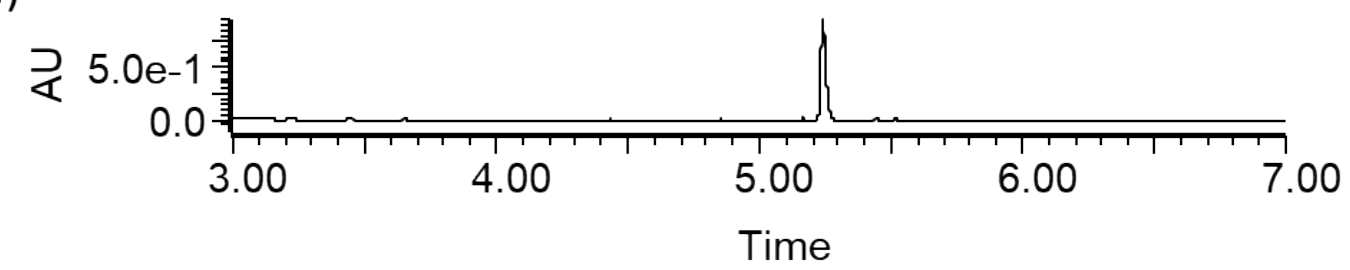


Figure S25. Construction of $n g n P 1$ deletion mutant, Nocardia sp. $\Delta N g n N 4$ (a) Depiction of $n g n N 4$, (b) Gel electrophoresis of PCR products. DNA templates were from wild type Nocardia sp. CS682 (W3), deletion mutant Nocardia sp. $\Delta$ NgnN4 (D3) and marker (M3).

(a)

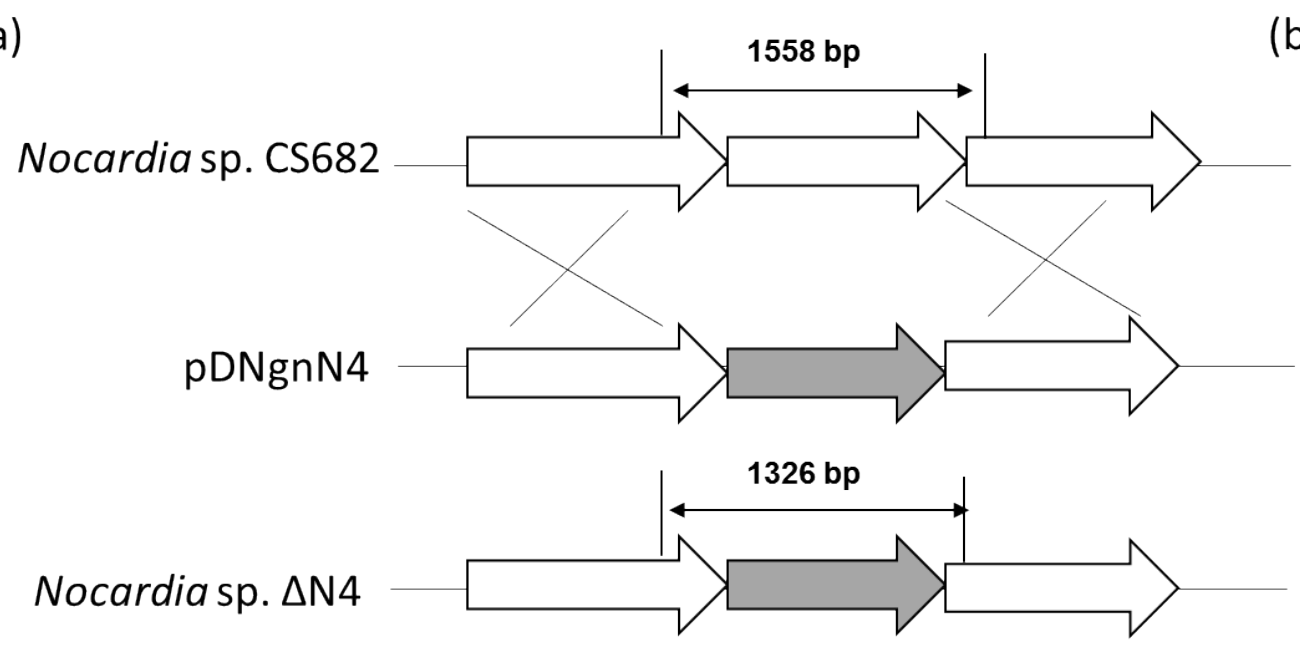

(b)

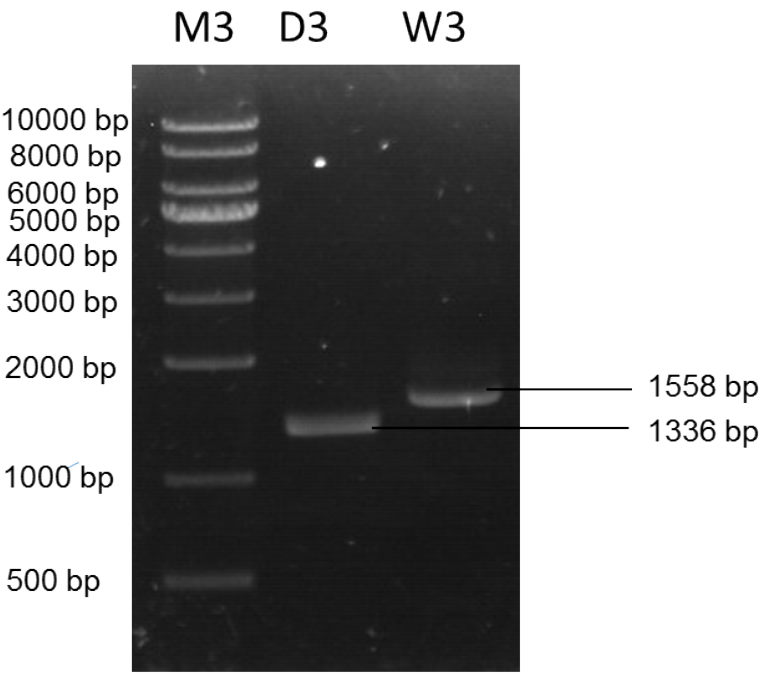


Figure S26. HPLC chromatogram of extract from different strains (a) Wild type, Nocardia sp. CS682, (b) deletion mutant Nocardia sp. $\triangle \mathrm{NgnM}$ and (c) complementation strain Nocardia sp. $\Delta \mathrm{NgnM} / \mathrm{p} 18 \mathrm{NgnM}$
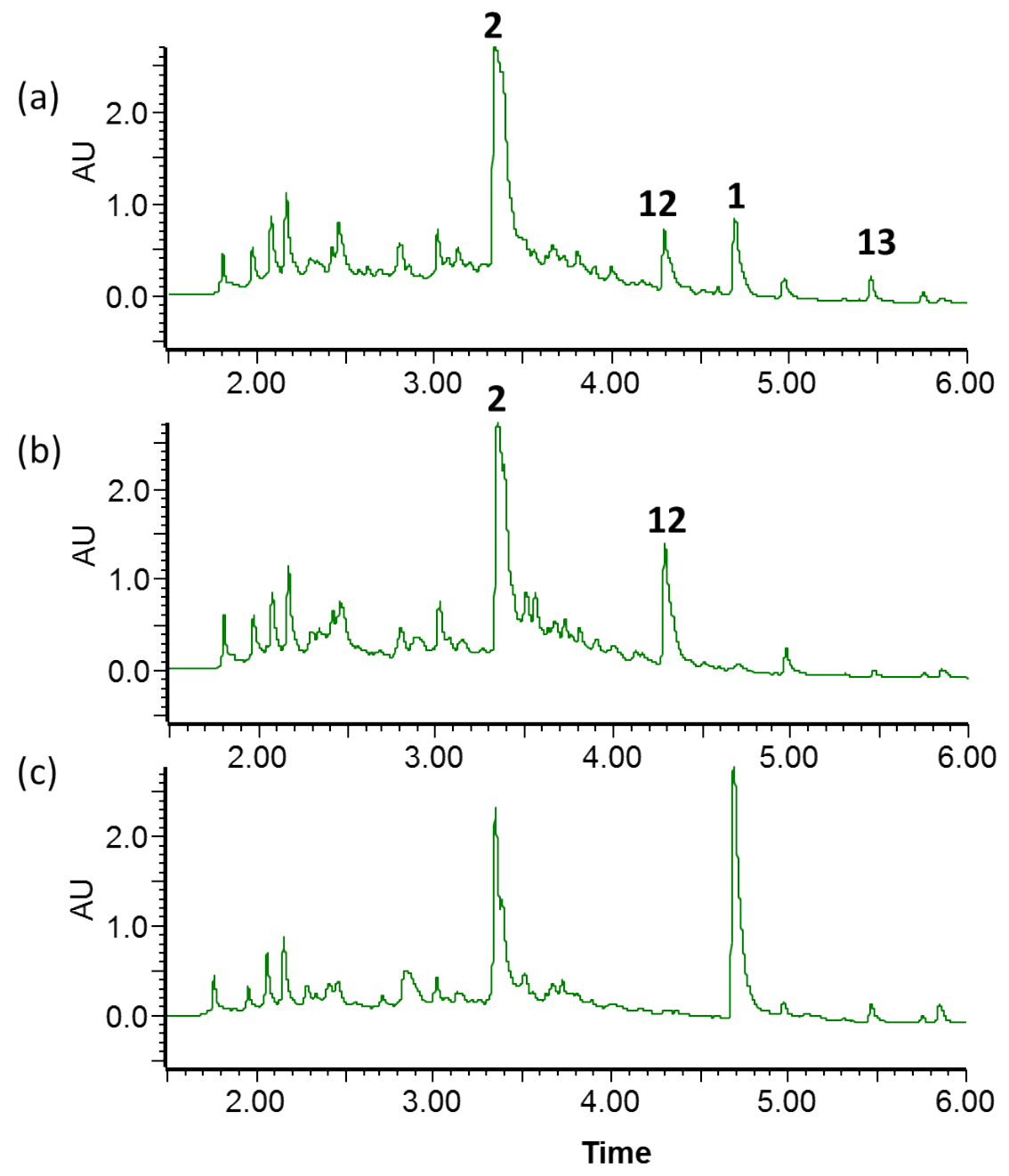


\section{References:}

1. Mishra, R.; Dhakal, D.; Han, J.M.; Lim, H.N.; Jung, H.J.; Yamaguchi, T.; Sohng, J.K. Production of a Novel Tetrahydroxynaphthalene (THN) Derivative from Nocardia sp. CS682 by Metabolic Engineering and Its Bioactivities. Molecules, 2019, 24, 244.

2. Tang, X.; Li, J.; Millán-Aguiñaga, N.; Zhang, J.J.; O’Neill, E.C.; Ugalde, J.A.; Jensen, P.R.; Mantovani, S.M.; Moore, B.S. Identification of Thiotetronic Acid Antibiotic Biosynthetic Pathways by Target-directed Genome Mining. ACS Chem Biol. 2015.10, 2841-2849.

3. Kouprina, N.; Larionov, V. Selective isolation of genomic loci from complex genomes by transformation-associated recombination cloning in the yeast Saccharomyces cerevisiae. Nat Protoc 2008, 3, 371-377.

4. Sohng, J.K.; Yamaguchi, T.; Seong, C.N.; Baik, K.S.; Park, S.C.; Lee, H.J.; Jang, S.Y.; Simkhada, J.R.; Yoo, J.C. Production, isolation and biological activity of nargenicin from Nocardia sp. CS682. Arch. Pharmacal. Res. 2008, 31, pp.1339-1345.

5. Jung, W.S.; Han, A.R.; Hong, J.S.J.; Park, S.R.; Choi, C.Y.; Park, J.W.; Yoon, Y.J. Bioconversion of 12-, 14-, and 16-membered ring aglycones to glycosylated macrolides in an engineered strain of Streptomyces venezuelae. Appl Microbiol Biotechnol. 2007, 76, 1373-1381

6. Bierman, M.; Logan, R.; O'brien, K.; Seno, E.T.; Rao, R.N.; Schoner, B.E. Plasmid cloning vectors for the conjugal transfer of DNA from Escherichia coli to Streptomyces spp. Gene,1992, 116,43-49.

7. Kelly, W.L;Townsend, C.A. Mutational analysis of nocK and nocL in the nocardicin a producer Nocardia uniformis. $J$ Bacteriol. 2005, 187,739-746.

8. Dhakal, D.; Chaudhary, A.K.; Yi, J.S.; Pokhrel, A.R.; Shrestha, B.; Parajuli, P.; Shrestha, A.; Yamaguchi, T.; Jung,H.J.; Kim, S.Y.; Kim, B.G. Enhanced production of nargenicin A1 and creation of a novel derivative using a synthetic 
biology platform. Appl. Microbiol. Biotechnol. 2016, 100, 9917-9931. 